\title{
The effect of biofeedback interventions on pain, overall symptoms, quality of life and physiological parameters in patients with pelvic pain
}

\section{A systematic review}

\author{
Barbara Wagner (D) - Margarete Steiner (D) Dominikus Franz Xaver Huber $(\mathbb{D}) \cdot$ Richard Crevenna $(\mathbb{D}$ \\ Received: 23 November 2020 / Accepted: 29 January 2021 / Published online: 22 March 2021 \\ (C) The Author(s) 2021
}

\begin{abstract}
Summary
Background Biofeedback is recognized as an effective additive method for treating certain phenotypes of chronic pelvic pain syndrome and is a therapeutic option in other pelvic pain conditions. This review aims to evaluate evidence from the literature with a focus on the effect of biofeedback on pain reduction, overall symptom relief, physiological parameters and quality of life.

Methods A systematic literature search was conducted using the databases PubMed, MEDLINE, Embase, Cochrane Library and PEDro from inception to July 2020. Data were tabulated and a narrative synthesis was carried out, since data heterogeneity did not allow a meta-analysis. The PEDro scale and the McMaster Critical Review Form-Quantitative Studies were applied to assess risk of bias.

Results Out of 651 studies, 37 quantitative studies of primary research evaluating pelvic pain conditions in male and female adults and children were included. They covered biofeedback interventions on anorectal disorders, chronic prostatitis, female chronic pelvic pain conditions, urologic phenotypes in children and adults and a single study on low back pain. For anorectal disorders, several landmark studies demonstrate the efficacy of biofeedback. For other subtypes of chronic pelvic pain conditions there is tentative evidence that biofeedback-assisted training has a positive effect on pain reduction, overall symptoms relief and quality of life. Certain factors have
\end{abstract}

\footnotetext{
Dr. B. Wagner · Mag. M. Steiner · Dr. D. F. X. Huber . Univ. Prof. Dr. R. Crevenna, MBA MMSc ( $₫)$ Department of Physical Medicine, Rehabilitation and Occupational Medicine, Medical University of Vienna, Waehringer Guertel 18-20, 1090 Vienna, Austria richard.crevenna@meduniwien.ac.at
}

been identified that might be relevant in improving treatment success.

Conclusions For certain indications, biofeedback has been confirmed to be an effective treatment. For other phenotypes, promising findings should be further investigated in robust and well-designed randomized controlled trials.

Keywords Pelvic floor dysfunction · Pelvic floor dyssynergia $\cdot$ EMG $\cdot$ Electromyography $\cdot$ Manometry

\section{Introduction}

Biofeedback therapy is an instrument-based learning process employing operant conditioning. Autonomic and neuromuscular activity is measured and visual, acoustic and verbal feedback is provided to promote the acquisition of self-control over physiological processes, which are otherwise outside awareness or under less voluntary control [1].

Pelvic pain is perceived in pelvis-related structures and organs of either men or women and may be acute or chronic. In terms of chronic pelvic pain (CPP), there is no generally accepted definition. It can be subdivided into conditions with well-defined classical pathology and those with no obvious pathology-the chronic pelvic pain syndromes (CPPS). The European Association of Urology (EAU) describes CPPS as the occurrence of CPP with no proven infection or other obvious local pathology accounting for the pain, continuous or recurrent for at least 6 months. It is often associated with symptoms suggestive of lower urinary tract, sexual, bowel, gynecological or pelvic floor dysfunction and with negative cognitive, behavioral, sexual or emotional consequences [2].

Chronic pelvic pain is a common pain condition with a worldwide prevalence of $2.1-26.6 \%$ for noncyclic pain in women [3-5] and $2.2-9.7 \%$ in men [6]. 
Up to $85 \%$ of women with CPP have dysfunction of the musculoskeletal system, including spasm of the levator ani muscle [7]. Myofascial pelvic pain is a major component of CPP which is not always properly identified by healthcare providers [8]. It may be a primary or contributing source of CPP [8]. Its hallmark diagnostic indicators are myofascial trigger points in the pelvic floor musculature that refer pain to adjacent sites [8]. They are thought to occur in response to acute and chronic physical or psychosocial stress or trauma [9].

The pathophysiology of CPP is not well understood. Treatment is therefore often unsatisfactory and limited to symptom relief [7]. Several nonsurgical strategies exist that include medical, psychological, cognitive, behavioral, complementary and physical therapy $[5,7,10]$. In the case of myofascial pelvic pain in particular, a multidisciplinary team of specialists [8] and a multimodal treatment strategy are warranted. In a large proportion of patients, treatment does not necessarily result in pain relief. CPP therefore carries a significant physical, mental, and social burden for patients and puts a heavy burden on healthcare systems worldwide. Increased medical attention to identify and test effective treatment strategies is warranted $[5,7,10,11]$.

Biofeedback seems to be a promising adjuvant tool in the cognitive-behavioral treatment of somatoform disorders because it aims to enhance control over the psychophysiological processes that may be involved in these conditions [1]. Biofeedback is also one of several effective physical therapy techniques used to treat myofascial pelvic pain [8]. The recent EAU guidelines 2019 on CPP state that biofeedback is the preferred treatment for chronic anal pain and can improve the outcome of myofascial therapy as an adjuvant to muscle exercises in patients with hypertonic pelvic floor dysfunction [2]. It is considered a treatment option in type III chronic prostatitis according to the National Institutes of Health (NIH) classification [10].

Previous systematic reviews have evaluated the evidence of physiotherapy interventions in general in the management of CPP [5, 12, 13]. One review focused on the effect of biofeedback on improving symptoms of pelvic floor dysfunction in 2008 [14]. The primary aim of our review was to evaluate the effect of biofeedback interventions on subjective outcome pain, overall symptom improvement and quality of life in patients with acute or chronic pelvic pain conditions. A secondary aim was to investigate whether biofeedback interventions improved physiological parameters indicative of pelvic floor muscle tone and/or general relaxation.

Table 1 Inclusion and exclusion criteria

\begin{tabular}{|c|c|c|}
\hline & Inclusion criteria & Exclusion criteria \\
\hline \multirow[t]{3}{*}{$\begin{array}{l}\text { Study design, } \\
\text { comparison }\end{array}$} & $\begin{array}{l}\text { Any quantitative study type of primary research with exception of } \\
\text { case studies/case series }<10 \text { participants; retrospective studies are } \\
\text { included }\end{array}$ & $\begin{array}{l}\text { Reviews, cross-sectional studies, case reports/case series }<10 \text { par- } \\
\text { ticipants, conference papers and abstracts, book chapters, editorials }\end{array}$ \\
\hline & \multirow{2}{*}{$\begin{array}{l}\text { Control interventions may include treatment as usual, no treatment, } \\
\text { surgery, medicinal treatment, physical therapy modalities or placebo } \\
\text { treatment }\end{array}$} & Unconcluded studies, studies with missing outcome data \\
\hline & & $\begin{array}{l}\text { Studies that were published in languages other than English and Ger- } \\
\text { man }\end{array}$ \\
\hline \multirow[t]{4}{*}{ Participants } & $\begin{array}{l}\text { People with chronic pelvic pain according to the guidelines on chronic } \\
\text { pelvic pain [2] including constipation (conditions with overactive pelvic } \\
\text { floor dysfunction) }\end{array}$ & \multirow[t]{4}{*}{$\begin{array}{l}\text { Pelvic organ prolapse, fecal or urinary incontinence (hypotonic pelvic } \\
\text { floor dysfunction) }\end{array}$} \\
\hline & People with acute pelvic pain & \\
\hline & Males and females & \\
\hline & Children, adolescents, adults & \\
\hline \multirow[t]{3}{*}{ Interventions } & $\begin{array}{l}\text { Biofeedback as a sole intervention or as a significant component of } \\
\text { a multimodal intervention }\end{array}$ & $\begin{array}{l}\text { No BFB-assisted training performed/BFB was not a relevant component } \\
\text { of the treatment }\end{array}$ \\
\hline & \multirow[t]{2}{*}{ Clinical (in/outpatient) setting or home-based training } & $\begin{array}{l}\text { If only a subgroup of the study population received BFB: studies were } \\
\text { excluded if the number of subjects in the BFB subgroup or the outcome } \\
\text { results of this subgroup were not stated }\end{array}$ \\
\hline & & $\begin{array}{l}\text { Insufficiently documented BFB intervention: no information on the } \\
\text { training extent (frequency, number of sessions or duration of a single } \\
\text { session) or the mode of application }\end{array}$ \\
\hline \multirow[t]{2}{*}{$\begin{array}{l}\text { Outcome } \\
\text { measures }\end{array}$} & $\begin{array}{l}\text { Primary outcome: } \\
\text { - Pain intensity } \\
\text { - Overall symptom improvement } \\
\text { - Quality of life }\end{array}$ & \multirow[t]{2}{*}{-} \\
\hline & $\begin{array}{l}\text { Secondary outcome: } \\
\text { - Physiological parameters, indicative of pelvic floor muscle tone or } \\
\text { general relaxation }\end{array}$ & \\
\hline
\end{tabular}


Table 2 Study characteristics

\begin{tabular}{|c|c|c|c|c|c|c|}
\hline Study & Country & $\begin{array}{l}\text { Study design } \\
\text { (details: see } \\
\text { Table 8) }\end{array}$ & $\begin{array}{l}n \text { of IG receiv- } \\
\text { ing BFB ( } n \text { of } \\
\text { whole study } \\
\text { participants) }\end{array}$ & $\begin{array}{l}\text { Drop-outs } \\
\text { at last f/u/ } \\
\text { excluded } \\
\text { from analysis }\end{array}$ & Diagnose(s), symptoms: & Conclusions by authors of respective papers \\
\hline \multicolumn{7}{|c|}{ Anorectal pain syndrome } \\
\hline $\begin{array}{l}\text { Chiarioni et } \\
\text { al. } 2010 \text { [16] }\end{array}$ & Italy & $\begin{array}{l}\text { RCT }(3 \text { arm): } \\
3 \text { different in- } \\
\text { terventions } \\
\text { compared }\end{array}$ & $52(157)$ & n.a. & $\begin{array}{l}\text { Levator ani syndrome } \\
\text { (constipation excluded) }\end{array}$ & $\begin{array}{l}\text { BFB is superior to EGS and levator ani massage in pain } \\
\text { relief. Improvements maintained for } 12 \text { months. Only } \\
\text { patients with tenderness on rectal examination benefit. } \\
\text { Pathophysiology of levator ani syndrome pathophysiology } \\
\text { is similar to dyssynergic defecation type constipation }\end{array}$ \\
\hline $\begin{array}{l}\text { Heah et al. } \\
1997[17]\end{array}$ & $\begin{array}{l}\text { Singa- } \\
\text { pore }\end{array}$ & $\begin{array}{l}\text { Non-RCT, single- } \\
\text { group (prospec- } \\
\text { tive) }\end{array}$ & $16(16)$ & $\begin{array}{l}0 / 16 \text { (post } \\
\text { treatment); } \\
\text { n.a. (later } \\
\text { f/u) }\end{array}$ & Levator ani syndrome & $\begin{array}{l}\text { Although BFB had a negligible effect on anorectal physi- } \\
\text { ologic measurements, it was effective in pain relief, with } \\
\text { no side effects }\end{array}$ \\
\hline $\begin{array}{l}\text { Ger et al. } \\
1993[18]\end{array}$ & USA & $\begin{array}{l}\text { Non-RCT, } 3 \text { arm, } \\
\text { non-randomized } \\
\text { (prospective) }\end{array}$ & $14(60)$ & $22 / 60$ & $\begin{array}{l}\text { Chronic intractable rec- } \\
\text { tal pain } \pm \text { coccygo- } \\
\text { dynia } \pm \text { constipation/ } \\
\text { dyschezia }(n=34 / 60)\end{array}$ & $\begin{array}{l}\text { BFB vs. EGS vs. epidural steroid caudal block: EGS and } \\
\text { BFB had almost the same poor results ( }>50 \% \text { were re- } \\
\text { fractory); No significant differences in rates of success or } \\
\text { failure after any of the } 3 \text { therapeutic options, regardless } \\
\text { of whether the option was a primary, secondary, or ter- } \\
\text { tiary choice. Associated historic factors or abnormalities } \\
\text { in anorectal physiologic studies did not influence results }\end{array}$ \\
\hline $\begin{array}{l}\text { Gilliland et al. } \\
\text { 1997a [19] }\end{array}$ & USA & $\begin{array}{l}\text { Non-RCT (retro- } \\
\text { spective) }\end{array}$ & $86(86)$ & $11 / 86$ & $\begin{array}{l}\text { Chronic intractable rec- } \\
\text { tal pain } \pm \text { constipation } \\
(n=30 / 86)\end{array}$ & $\begin{array}{l}\text { EMG-based BFB can produce alleviation of idiopathic } \\
\text { rectal pain. Outcome was significantly improved in pa- } \\
\text { tients who completed the treatment schedule compared } \\
\text { to those who self-discharged. Outcome was not influ- } \\
\text { enced by patients' ages, duration of symptoms or prior } \\
\text { history of surgery and was not significantly related to the } \\
\text { presence of paradoxical puborectalis contraction (EMG or } \\
\text { defecography) }\end{array}$ \\
\hline $\begin{array}{l}\text { Grimaud et } \\
\text { al. } 1991 \text { [20] }\end{array}$ & France & $\begin{array}{l}\text { Non-RCT, single- } \\
\text { group (prospec- } \\
\text { tive)+ cross- } \\
\text { sectional }\end{array}$ & $\begin{array}{l}12(24) \\
12 \text { healthy }\end{array}$ & $0 / 12$ & $\begin{array}{l}\text { Chronic idiopathic anal } \\
\text { pain } \pm \text { constipation } \\
(n=9 / 12)\end{array}$ & $\begin{array}{l}\text { Chronic idiopathic anal pain is associated with abnor- } \\
\text { mal anorectal manometric profiles ( } \uparrow \text { anal canal resting } \\
\text { pressure), probably resulting from a dysfunctioning of } \\
\text { the striated external anal sphincter. BFB is an effective } \\
\text { treatment for chronic idiopathic anal pain. Anorectal pain } \\
\text { disappeared after a mean of } 8 \text { BFB sessions }\end{array}$ \\
\hline \multicolumn{7}{|c|}{ Constipation, dyssynergic defecation } \\
\hline $\begin{array}{l}\text { Chiarioni et } \\
\text { al. } 2006 \text { [21] }\end{array}$ & Italy & $\begin{array}{l}\text { RCT ( } 2 \text { arm: } \\
\text { intervention } \\
\text { vs. different } \\
\text { intervention) }\end{array}$ & $54(109)$ & $\begin{array}{l}14 / 109 \\
\text { (10/54 in } \\
\text { BFB group) }\end{array}$ & $\begin{array}{l}\text { Normal transit constipa- } \\
\text { tion due to PFD (Rome II } \\
\text { criteria) }\end{array}$ & $\begin{array}{l}5 \times 30 \text { min BFB sessions are more effective than continu- } \\
\text { ous polyethylene glycol in PF dyssynergia (major improve- } \\
\text { ment in } 80 \% \text { ), benefits last at least } 2 \text { years. BFB should } \\
\text { become the treatment of choice PFD. Predictors of bet- } \\
\text { ter response to BFB: sensation of incomplete or blocked } \\
\text { evacuation, straining with bowel movements; predictors } \\
\text { of poorer response: digital facilitation of defecation }\end{array}$ \\
\hline $\begin{array}{l}\text { Koutsomanis } \\
\text { et al. } 1994 \\
\text { [22] }\end{array}$ & Italy & $\begin{array}{l}\text { Non-RCT, single- } \\
\text { group (prospec- } \\
\text { tive) }\end{array}$ & $54(109)$ & $10 / 30$ & Idiopathic constipation & $\begin{array}{l}\sim 50 \% \text { of patients were helped by } 2-6 \text { BFB sessions, im- } \\
\text { provement persisted for } \geq 6-12 \text { months. Both types of } \\
\text { PF incoordination (inability to relax on defecation and in- } \\
\text { ability to strain effectively) improved. No clear correlation } \\
\text { between change in transit rate and symptomatic outcome }\end{array}$ \\
\hline $\begin{array}{l}\text { Chiotakakou- } \\
\text { Faliakou et } \\
\text { al. } 1998 \text { [23] }\end{array}$ & UK & $\begin{array}{l}\text { Non-RCT (retro- } \\
\text { spective) }\end{array}$ & $30(30)$ & $0 / 100$ & $\begin{array}{l}\text { Chronic idiopathic slow } \\
\text { and normal transit consti- } \\
\text { pation }\end{array}$ & $\begin{array}{l}\text { BFB is an effective long-term treatment for the majority } \\
\text { of patients with idiopathic constipation unresponsive to } \\
\text { traditional treatment ( }>50 \% \text { improved). Patients with } \\
\text { slow/normal transit, males/females, with/without para- } \\
\text { doxical PF contraction benefited equally. Anorectal test- } \\
\text { ing did not predict outcome }\end{array}$ \\
\hline $\begin{array}{l}\text { Battaglia et } \\
\text { al. } 2004 \text { [24] }\end{array}$ & UK & $\begin{array}{l}\text { Non-RCT, single- } \\
\text { group (prospec- } \\
\text { tive) }\end{array}$ & $100(100)$ & n.a. & $\begin{array}{l}\text { Chronic constipation } \\
\text { (Rome II criteria) }\end{array}$ & $\begin{array}{l}\text { Patients with PFD are likely to have continued benefit } \\
\text { from BFB, whereas its effects on slow-transit constipa- } \\
\text { tion seems to be maximal in short-term course. Anorec- } \\
\text { tal manometric variables remained unchanged (apart } \\
\text { from sensation threshold } \downarrow \text { in PFD group, maximum } \\
\text { rectal tolerable volume } \downarrow \text { in slow-transit group) }\end{array}$ \\
\hline $\begin{array}{l}\text { Wang et al. } \\
2003 \text { [25] }\end{array}$ & Italy & $\begin{array}{l}\text { Non-RCT, single- } \\
\text { group (prospec- } \\
\text { tive) }\end{array}$ & $24(24)$ & n.a. & $\begin{array}{l}\text { Chronic idiopathic consti- } \\
\text { pation (Rome II criteria) }\end{array}$ & $\begin{array}{l}\text { BFB has a long-term effect with no side effects for most } \\
\text { patients (62.5\%) with chronic idiopathic constipation } \\
\text { unresponsive to traditional treatment. Patients with slow/ } \\
\text { normal transit, with/without paradoxical PF contraction } \\
\text { benefited equally. The psychological status rather than } \\
\text { anorectal test could predict outcome. The efficacy of the } \\
\text { two modes of BFB was similar }\end{array}$ \\
\hline
\end{tabular}


Table 2 (Continued)

\begin{tabular}{|c|c|c|c|c|c|c|}
\hline Study & Country & $\begin{array}{l}\text { Study design } \\
\text { (details: see } \\
\text { Table 8) }\end{array}$ & $\begin{array}{l}n \text { of IG receiv- } \\
\text { ing BFB ( } n \text { of } \\
\text { whole study } \\
\text { participants) }\end{array}$ & $\begin{array}{l}\text { Drop-outs } \\
\text { at last f/u/ } \\
\text { excluded } \\
\text { from analysis }\end{array}$ & Diagnose(s), symptoms: & Conclusions by authors of respective papers \\
\hline $\begin{array}{l}\text { Ba-Bai-Ke- } \\
\text { Re et al. } \\
2014 \text { [26] }\end{array}$ & China & $\begin{array}{l}\text { RCT }(2 \text { arm: } 2 \\
\text { different inter- } \\
\text { ventions) }\end{array}$ & $50(50)$ & $0 / 44$ & $\begin{array}{l}\text { Chronic obstructive con- } \\
\text { stipation (Rome III criteria) }\end{array}$ & $\begin{array}{l}\text { Manometric BFB-guided PF exercise is superior to oral } \\
\text { polyethylene glycol for obstructive defecation for improv- } \\
\text { ing overall symptoms, pain at defecation, quality of life }\end{array}$ \\
\hline $\begin{array}{l}\text { Roy et al. } \\
2000 \text { [27] }\end{array}$ & China & $\begin{array}{l}\text { Non-RCT (retro- } \\
\text { spective) }\end{array}$ & $44(88)$ & n.a. & $\begin{array}{l}\text { Chronic idiopathic con- } \\
\text { stipation (no surgery: } \\
n=25 / 78 \text {, hysterectomy } \\
\text { and no change in bowel } \\
\text { function: } n=27 / 78 \text {, hys- } \\
\text { terectomy subjectively led } \\
\text { to constipation: } n=26 / 78 \text { ) }\end{array}$ & $\begin{array}{l}\text { The majority of patients complaining of constipation in- } \\
\text { duced by hysterectomy subjectively respond to behavioral } \\
\text { treatment, in a similar proportion to those with idiopathic } \\
\text { constipation. Physiological testing did not predict out- } \\
\text { come }\end{array}$ \\
\hline $\begin{array}{l}\text { Chiarioni et } \\
\text { al. } 2005 \text { [28] }\end{array}$ & UK & $\begin{array}{l}\text { Non-RCT, single } \\
\text { group (prospec- } \\
\text { tive) }\end{array}$ & $78(78)$ & $7 / 52$ & $\begin{array}{l}\text { Chronic idiopathic consti- } \\
\text { pation (PFD: } n=34 / 52 \text {, } \\
\text { slow transit only: } \\
n=12 / 52,1-2 \text { criteria } \\
\text { for PFD: } n=6 / 52 \text { ) }\end{array}$ & $\begin{array}{l}\text { BFB is an effective treatment for PFD but not slow-transit } \\
\text { constipation. Improvements were maintained at f/u } \\
24 \text { months. BFB eliminated dyssynergia in } 91 \% \text { and } \\
\text { enabled } 85 \% \text { to defecate the balloon. Success was } \\
\text { predicted by PFD, milder constipation, and less frequent } \\
\text { abdominal pain at baseline }\end{array}$ \\
\hline $\begin{array}{l}\text { Zhu et al. } \\
2011 \text { [29] }\end{array}$ & Italy & $\begin{array}{l}\text { Non-RCT single } \\
\text { group, observa- } \\
\text { tional }\end{array}$ & $41(41)$ & $5 / 41$ & $\begin{array}{l}\text { Functional constipation } \\
\text { with PFD (Rome III criteria) }\end{array}$ & $\begin{array}{l}\text { Before treatment, } 7 \text { Short Form-36 subscales (except } \\
\text { bodily pain) were significantly lower in people with PFD } \\
\text { than in healthy individuals. After BFB, all subcategories } \\
\text { except general health showed improvement surpassing } \\
\text { pretreatment baseline values and equalling those for } \\
\text { normal. The total Patient Assessment of Constipation } \\
\text { Quality of Life Questionnaire score also dramatically } \\
\text { improved as did all subscales }\end{array}$ \\
\hline $\begin{array}{l}\text { Gilliland et al. } \\
\text { 1997b [30] }\end{array}$ & USA & $\begin{array}{l}\text { Non-RCT (retro- } \\
\text { spective) }\end{array}$ & 194 (194) & $16 / 194$ & $\begin{array}{l}\text { Chronic constipa- } \\
\text { tion } \pm \text { concomitant rectal } \\
\text { pain }(n=30 / 194)\end{array}$ & $\begin{array}{l}\text { Success rate of BFB for constipated patients is less than } \\
\text { previously reported ( } 35 \% \text { complete success, } 13 \% \text { par- } \\
\text { tial success). Success rate } 1 \text { after } \geq 5 \text { sessions and was } \\
\text { significantly related to patient's willingness to complete } \\
\text { treatment and number of sessions attended. Neither pa- } \\
\text { tient age, sex, abnormalities in manometry nor duration } \\
\text { of symptoms significantly affected outcome }\end{array}$ \\
\hline $\begin{array}{l}\text { Parker et al. } \\
2019 \text { [31] }\end{array}$ & Canada & $\begin{array}{l}\text { Non-RCT (retro- } \\
\text { spective) }\end{array}$ & $130(130)$ & $38 / 168$ & $\begin{array}{l}\text { Chronic constipation + PFD } \\
(n=53 / 130) \text {, without } \\
\text { PFD }(n=3 / 130) \text {, fecal } \\
\text { incontinence }(n=49 / 130) \text {, } \\
\text { constipation + fecal incon- } \\
\text { tinence }(n=22 / 130) \text {, rectal } \\
\text { pain }(n=3 / 130)\end{array}$ & $\begin{array}{l}\text { In patients with chronic constipation due to PFD, over- } \\
\text { all response rate was } 69.8 \% \text {, only } 45.3 \% \text { had symp- } \\
\text { tomatic improvement. These patients are less likely to } \\
\text { have symptomatic response than those with fecal in- } \\
\text { continence. } 3 \text { BFB sessions are insufficient to manage } \\
\text { dyssynergic defecation }\end{array}$ \\
\hline \multicolumn{7}{|c|}{ Male chronic pelvic pain syndrome, urological chronic pelvic pain syndrome } \\
\hline $\begin{array}{l}\text { Clemens et } \\
\text { al. } 2000 \text { [32] }\end{array}$ & - & $\begin{array}{l}\text { Non-RCT, single } \\
\text { group }\end{array}$ & $19(19)$ & $3 / 19$ & $\begin{array}{l}\text { Nonbacterial male CPPS } \\
\text { [33] (NIH type IIIA prostati- } \\
\text { tis: } n=6 / 19, \text { NIH type IIIB } \\
\text { prostatitis: } n=13 / 19)\end{array}$ & $\begin{array}{l}\text { A formalized program of neuromuscular reeducation of } \\
\text { PF muscles with interval bladder training can provide } \\
\text { significant, durable improvement in objective measures } \\
\text { of pain, urgency, and frequency in patients with CPPS. } \\
\text { Detrusor instability, hypersensitivity to filling, or bladder- } \\
\text { sphincter pseudodyssynergia on pretreatment urody- } \\
\text { namic studies were not predictive of treatment results }\end{array}$ \\
\hline $\begin{array}{l}\text { Cornel et al. } \\
2005 \text { [34] }\end{array}$ & USA & $\begin{array}{l}\text { Non-RCT, single } \\
\text { group; observa- } \\
\text { tional }\end{array}$ & $33(33)$ & $\begin{array}{l}2 / 33(15 / 33 \\
\text { for EMG } \\
\text { values) }\end{array}$ & $\begin{array}{l}\text { Male CPPS (NIH type III } \\
\text { prostatitis) [33] }\end{array}$ & $\begin{array}{l}\text { BFB physical therapy and PF reeducation lead to a signif- } \\
\text { icant symptom improvement and decrease of PF muscle } \\
\text { tonus }\end{array}$ \\
\hline $\begin{array}{l}\text { Yang et al. } \\
2017 \text { [35] }\end{array}$ & $\begin{array}{l}\text { Nether- } \\
\text { lands }\end{array}$ & $\begin{array}{l}\text { Non-RCT (retro- } \\
\text { spective) }\end{array}$ & $22(50)$ & $5 / 50$ & $\begin{array}{l}\text { Male CPPS (NIH type IIIA, } \\
\text { IIIB prostatitis) [33] }\end{array}$ & $\begin{array}{l}\text { Both electromagnetic stimulation and EGS + BFB physical } \\
\text { therapy of PF muscle effectively reduce pain, increase } \\
\text { quality of life and improve urinary tract symptoms in re- } \\
\text { fractory male CPPS. EGS + BFB had additional benefits on } \\
\text { pain and QoL compared to electromagnetic stimulation } \\
\text { alone }\end{array}$ \\
\hline $\begin{array}{l}\text { He et al. } \\
2010[36]\end{array}$ & Taiwan & $\begin{array}{l}\text { Non-RCT (retro- } \\
\text { spective) }\end{array}$ & $21(21)$ & n.a. & $\begin{array}{l}\text { Nonbacterial male } \\
\text { CPPS + dysfunctional } \\
\text { voiding }\end{array}$ & $\begin{array}{l}\text { BFB had satisfactory short-term effects on patients with } \\
\text { dysfunctional voiding and chronic prostatitis. Urodynam- } \\
\text { ics could be used to help in the diagnosis and select the } \\
\text { most appropriate treatment }\end{array}$ \\
\hline
\end{tabular}


Table 2 (Continued)

\begin{tabular}{|c|c|c|c|c|c|c|}
\hline Study & Country & $\begin{array}{l}\text { Study design } \\
\text { (details: see } \\
\text { Table 8) }\end{array}$ & $\begin{array}{l}n \text { of IG receiv- } \\
\text { ing BFB ( } n \text { of } \\
\text { whole study } \\
\text { participants) }\end{array}$ & $\begin{array}{l}\text { Drop-outs } \\
\text { at last f/u/ } \\
\text { excluded } \\
\text { from analysis }\end{array}$ & Diagnose(s), symptoms: & Conclusions by authors of respective papers \\
\hline \multicolumn{7}{|c|}{ Female chronic pelvic pain } \\
\hline $\begin{array}{l}\text { Schmitt et al. } \\
2017[37]\end{array}$ & USA & $\begin{array}{l}\text { Non-RCT, single- } \\
\text { group (prospec- } \\
\text { tive) }\end{array}$ & $94(94)$ & $\begin{array}{l}0 / 94 \\
\text { dropouts, but } \\
\text { missing out- } \\
\text { come data } \\
(n: \text { Tables } 2, \\
3 \text { and } 4)\end{array}$ & $\begin{array}{l}\text { Pelvic pain or dyspareunia } \\
(n=29 / 94) \text {, defecatory } \\
\text { smptoms }(n=31 / 94) \text {, uri- } \\
\text { nary symptoms }(n=84 / 94)\end{array}$ & $\begin{array}{l}\text { An aggressive PF rehabilitation program including BFB } \\
\text { with vaginal EGS had a high rate of self-reported subjec- } \\
\text { tive success and satisfaction in patients with PF dysfunc- } \\
\text { tion }\end{array}$ \\
\hline $\begin{array}{l}\text { Glazer et al. } \\
1995 \text { [38] }\end{array}$ & USA & $\begin{array}{l}\text { Non-RCT, single- } \\
\text { group (prospec- } \\
\text { tive) }\end{array}$ & $33(33)$ & n.a. & $\begin{array}{l}\text { Vulvar vestibulitis syn- } \\
\text { drome }\end{array}$ & $\begin{array}{l}\text { PF muscle instability is a critical factor in pain associ- } \\
\text { ated with vulvar vestibulitis syndrome. A BFB-assisted } \\
\text { exercise program that stabilizes PF muscles significantly } \\
\text { reduces and, in some cases, eliminates symptoms. The } \\
\text { more the PF muscle stabilized, the more pain decreased, } \\
\text { the higher the initial pain, the higher the pain reduction. } \\
6 \text {-month f/u indicated maintenance of therapeutic bene- } \\
\text { fits }\end{array}$ \\
\hline $\begin{array}{l}\text { McKay et al. } \\
2001 \text { [39] }\end{array}$ & USA & $\begin{array}{l}\text { Non-RCT, single- } \\
\text { group (prospec- } \\
\text { tive) }\end{array}$ & $29(29)$ & $\begin{array}{l}\text { Monthly } \\
\text { f/u, } 25 / 29 \\
\text { (!) after } \\
11 \text { months }\end{array}$ & $\begin{array}{l}\text { Moderate-severe vulvar } \\
\text { vestibulitis syndrome }\end{array}$ & $\begin{array}{l}\text { EMG BFB of PF is an effective approach to vulvar vestibu- } \\
\text { litis. } 88.9 \% \text { reported negligible or mild pain after treat- } \\
\text { ment }\end{array}$ \\
\hline $\begin{array}{l}\text { Gentilcore- } \\
\text { Saulnier et al. } \\
2010 \text { [40] }\end{array}$ & Canada & $\begin{array}{l}\text { Non-RCT, single- } \\
\text { group (prospec- } \\
\text { tive)+ cross- } \\
\text { sectional }\end{array}$ & $11(22)$ & n.a. & $\begin{array}{l}\text { Provoked vestibulodynia } \pm \\
\text { constipation, dysmenor- } \\
\text { rhea, urinary frequency }\end{array}$ & $\begin{array}{l}\text { Women with provoked vestibulodynia showed altered } \\
\text { PF muscle behavior compared to controls, providing } \\
\text { empirical evidence of PF muscle dysfunction, especially } \\
\text { at the superficial layer. A physiotherapy rehabilitation } \\
\text { program targeting PF muscle dysfunction normalized PF } \\
\text { muscle behavior }\end{array}$ \\
\hline $\begin{array}{l}\text { Bendana et } \\
\text { al. } 2009 \text { [41] }\end{array}$ & USA & $\begin{array}{l}\text { Non-RCT (retro- } \\
\text { spective) }\end{array}$ & $52(52)$ & $\begin{array}{l}0 / 52 \\
\text { dropouts }^{a}\end{array}$ & $\begin{array}{l}\text { Painful PF spasm + urinary } \\
\text { urgency/frequency }\end{array}$ & $\begin{array}{l}\text { Combined transvaginal BFB, electrostimulation and be- } \\
\text { havioral therapy targeting PF relaxation demonstrated } \\
\text { statistically significant improvement in urinary symptoms } \\
\text { for up to } 3 \text { months. Further study for refractory patients is } \\
\text { warranted }\end{array}$ \\
\hline $\begin{array}{l}\text { Philips et al. } \\
1992 \text { [42] }\end{array}$ & Canada & $\begin{array}{l}\text { RCT (randomized } \\
\text { yoked design, } \\
\text { intervention vs. } \\
\text { different vs. no } \\
\text { intervention) }\end{array}$ & 10 & n.a. & $\begin{array}{l}\text { Urethral syn- } \\
\text { drome + functional urinary } \\
\text { incoordination (pelvic pain: } \\
n=11 / 30 \text {, dyspareunia: } \\
n=9 / 30 \text { ) }\end{array}$ & $\begin{array}{l}\text { Both EMG BFB and progressive muscle relaxation proved } \\
\text { effective in improving symptomology and psychological } \\
\text { state in patients with functional urinary incoordination }\end{array}$ \\
\hline $\begin{array}{l}\text { Hart et al. } \\
1981 \text { [43] }\end{array}$ & USA & $\begin{array}{l}\text { Non-RCT, } 2 \\
\text { arm: } 2 \text { different } \\
\text { interventions } \\
\text { (prospective) }\end{array}$ & $14(14)$ & $3 / 14$ & $\begin{array}{l}\text { Spasmodic dysmenorrhea } \\
\text { according to MSQ }\end{array}$ & $\begin{array}{l}\text { BFB and temperature training are effective in reducing } \\
\text { many of the painful symptoms of primary dysmenorrhea. } \\
\text { There was no significant difference between EMG and } \\
\text { temperature training }\end{array}$ \\
\hline $\begin{array}{l}\text { Bennink et al. } \\
1982[44]\end{array}$ & USA & $\begin{array}{l}\text { RCT ( } 3 \text { arm: } \\
\text { interven- } \\
\text { tion } \pm \text { different } \\
\text { intervention vs. } \\
\text { no intervention) }\end{array}$ & $5(15)$ & $0 / 15$ & $\begin{array}{l}\text { Primary dysmenorrhea } \\
\text { (spasmodic or congestive) }\end{array}$ & $\begin{array}{l}\text { Subjective reports indicated that the symptoms of dys- } \\
\text { menorrhea improved for the BFB group but not for the } \\
\text { relaxation or control groups }\end{array}$ \\
\hline $\begin{array}{l}\text { Vagedes et } \\
\text { al. } 2019 \text { [45] }\end{array}$ & $\begin{array}{l}\text { Ger- } \\
\text { many }\end{array}$ & $\begin{array}{l}\text { RCT (3 arm: } 2 \\
\text { different inter- } \\
\text { ventions vs. } \\
\text { standard care) }\end{array}$ & $20(60)$ & $\begin{array}{l}12 / 60(6 / 20 \\
\text { in BFB } \\
\text { group) }\end{array}$ & Primary dysmenorrhea & $\begin{array}{l}\text { Preliminary evidence suggests that rhythmical massage } \\
\text { might improve pain intensity after } 12 \text { weeks compared } \\
\text { to usual care. No significant differences were found } \\
\text { between heart rate variability-based home-BFB and the } \\
\text { control group }\end{array}$ \\
\hline $\begin{array}{l}\text { Starr et al. } \\
2013 \text { [46] }\end{array}$ & USA & $\begin{array}{l}\text { Non-RCT (retro- } \\
\text { spective) }\end{array}$ & 778 (778) & $97 / 778$ & $\begin{array}{l}\text { PF dysfunction (urinary: } \\
n=694 / 778 \text {, defecatory: } \\
n=187 / 778 \text {, pelvic pain: } \\
n=368 / 778 \text { ) }\end{array}$ & $\begin{array}{l}\text { Comprehensive PF rehabilitation including PF muscle } \\
\text { training, BFB, EGS, constipation management, behavioral } \\
\text { modification, incontinence devices, and pharmacotherapy } \\
\text { is effective in treating women with PF dysfunction }\end{array}$ \\
\hline $\begin{array}{l}\text { Lúcio et al. } \\
2014 \text { [47] }\end{array}$ & Brazil & $\begin{array}{l}\text { RCT, (3 arm: } \\
\text { multimodal + } \\
\text { sham vs. lo- } \\
\text { cal vs. distal } \\
\text { electrotherapy) }\end{array}$ & $30(30)$ & $10 / 30$ & $\begin{array}{l}\text { Woman with multiple scle- } \\
\text { rosis + sexual dysfunction }\end{array}$ & $\begin{array}{l}\text { PF muscle training with EMG BFB-alone or combined } \\
\text { with intravaginal EGS or transcutaneous tibial nerve } \\
\text { stimulation-contributes to the improvement of sexual } \\
\text { dysfunction in patients with multiple sclerosis }\end{array}$ \\
\hline $\begin{array}{l}\text { Aalaie et al. } \\
2020 \text { [48] }\end{array}$ & Iran & $\begin{array}{l}\text { RCT ( } 2 \text { arm: } 2 \\
\text { different inter- } \\
\text { ventions) }\end{array}$ & $11(22)$ & $\begin{array}{l}1 / 22 \text { (in BFB } \\
\text { group) }\end{array}$ & $\begin{array}{l}\text { Female sexual pain dys- } \\
\text { function (DSM- } 5 \text { criteria } \\
\text { [49, 50], FSFI) + stress } \\
\text { urinary incontinence }\end{array}$ & $\begin{array}{l}\text { Both BFB and EGS increased the FSFI score. Both in- } \\
\text { terventions decreased pain during vaginal penetration } \\
\text { similarly. To improve sexual function, women undergoing } \\
\text { BFB seem to benefit more than those receiving EGS }\end{array}$ \\
\hline
\end{tabular}




\begin{tabular}{|c|c|c|c|c|c|c|}
\hline Study & Country & $\begin{array}{l}\text { Study design } \\
\text { (details: see } \\
\text { Table 8) }\end{array}$ & $\begin{array}{l}n \text { of IG receiv- } \\
\text { ing BFB ( } n \text { of } \\
\text { whole study } \\
\text { participants) }\end{array}$ & $\begin{array}{l}\text { Drop-outs } \\
\text { at last f/u/ } \\
\text { excluded } \\
\text { from analysis }\end{array}$ & Diagnose(s), symptoms: & Conclusions by authors of respective papers \\
\hline \multicolumn{7}{|c|}{ Chronic pelvic pain in children } \\
\hline $\begin{array}{l}\text { Hoebeke et } \\
\text { al. } 2004 \text { [51] }\end{array}$ & Belgium & $\begin{array}{l}\text { Non-RCT, single- } \\
\text { group (prospec- } \\
\text { tive) }\end{array}$ & $21(21)$ & n.a. & $\begin{array}{l}\text { PF spasms } \pm \text { detrusor } \\
\text { hyperactivity }(n=13 / 21) \\
\pm \text { dysfunctional voiding } \\
(n=5 / 21), \pm \text { constipation } \\
(n=8 / 21)\end{array}$ & $\begin{array}{l}\text { Pelvic floor spasms in children (which can be secondary } \\
\text { to detrusor overactivity) respond well to pelvic floor relax- } \\
\text { ation therapy }\end{array}$ \\
\hline $\begin{array}{l}\text { Ebiloglu et al. } \\
2016 \text { [52] }\end{array}$ & Turkey & $\begin{array}{l}\text { Non-RCT (retro- } \\
\text { spective) }\end{array}$ & $136(136)$ & n.a. & $\begin{array}{l}\text { Overactive bladder syn- } \\
\text { drome }\end{array}$ & $\begin{array}{l}\text { BFB can be thought of as the first-line treatment op- } \\
\text { tion when standard urotherapy fails. Success rate was } \\
53 \% \text { for urgency, } 69 \% \text { for dysuria. The mean LUTSS } \\
\text { significantly improved after BFB. Patients without hold- } \\
\text { ing maneuvers, daytime incontinence and enuresis had } \\
\text { better recovery compared to the opposites }\end{array}$ \\
\hline $\begin{array}{l}\text { Ergin et al. } \\
2016 \text { [53] }\end{array}$ & Turkey & $\begin{array}{l}\text { Non-RCT, single- } \\
\text { group (prospec- } \\
\text { tive) + cross- } \\
\text { sectional }\end{array}$ & $\begin{array}{l}52(100) \\
48 \text { healthy }\end{array}$ & $13 / 52$ & $\begin{array}{l}\text { Dysfunctional voiding } \\
(n=52 / 100),+ \text { overac- } \\
\text { tive bladder syndrome } \\
(n=27 / 52)\end{array}$ & $\begin{array}{l}\text { Urinary nerve growth factor (UNGF) levels were higher in } \\
\text { children with dysfunctional voiding and decreased after } \\
\text { BFB. UNGF levels could be used for the diagnosis and the } \\
\text { assessment of BFB success in these children }\end{array}$ \\
\hline $\begin{array}{l}\text { Li et al. } 2006 \\
\text { [54] }\end{array}$ & China & $\begin{array}{l}\text { Non-RCT, single- } \\
\text { group (prospec- } \\
\text { tive)+ cross- } \\
\text { sectional }\end{array}$ & $\begin{array}{l}25(40) \\
15 \text { healthy }\end{array}$ & $0 / 25$ & $\begin{array}{l}\text { Pubertal chronic pro- } \\
\text { statitis, NIH type II } \\
(n=1 / 25) \text {, IIIA }(n=3 / 25) \\
\text { IIIB }(n=21 / 25)\end{array}$ & $\begin{array}{l}\text { The main type of chronic prostatitis during puberty is } \\
\text { IIIB; the dominating symptom is a voiding disorder. The } \\
\text { impact on life and psychological effects are substantial. } \\
\text { Pubertal boys with chronic prostatitis have PF dysfunction } \\
\text { and several abnormal urodynamic values. The effect of } \\
\text { BFB in pubertal chronic prostatitis is satisfactory }\end{array}$ \\
\hline \multicolumn{7}{|c|}{ Musculoskeletal, low back pain, myofascial pain } \\
\hline $\begin{array}{l}\text { Kent et al. } \\
2015 \text { [55] }\end{array}$ & $\begin{array}{l}\text { Den- } \\
\text { mark }\end{array}$ & $\begin{array}{l}\text { RCT ( } 2 \text { arm: } \\
\text { intervention + } \\
\text { standard care } \\
\text { vs. placebo+ } \\
\text { standard care) }\end{array}$ & $58(112)$ & $19 / 112$ & $\begin{array}{l}\text { Subacute-chronic low } \\
\text { back pain }\end{array}$ & $\begin{array}{l}\text { Individualized movement retraining using motion-sensor } \\
\text { biofeedback resulted in significant and sustained im- } \\
\text { provements in low back pain. This pilot trial also refined } \\
\text { the procedures and sample size requirements for a fully } \\
\text { powered RCT }\end{array}$ \\
\hline \multicolumn{7}{|c|}{$\begin{array}{l}\text { BFB biofeedback, CPPS chronic pelvic pain syndrome, CP/CPPS chronic prostatitis/chronic pelvic pain syndrome, DSM-5 criteria diagnostic and statistical man- } \\
\text { ual of mental disorders, EGS electrogalvanic stimulation, EMG electromyography, FSFI female sexual function index [56], f/u follow-up, IG intervention goup, } \\
\text { LUTSS lower urinary tract symptom score [57], MSQ menstural Symptom questionnaire (MSQ) [58], NIH National Institute of Health, NIH-CPS/ National Institute } \\
\text { of Health - chronic prostatitis symptom index score, PF pelvic floor, PFD pelvic floor dyssynergia, } \downarrow \text { significant decrease, i significant increase, RCT randomized } \\
\text { controlled trial, vs. versus } \\
\text { amissing outcome data: } n=31 / 52 \text { for American Urological Association (AUA) symptom score [59], } n=39 / 52 \text { for visual analog scale (VAS) [60]) symptom severity/ } \\
\text { effect daily life }\end{array}$} \\
\hline
\end{tabular}

\section{Methods}

\section{Protocol and registration}

A systematic review of the existing scientific literature was conducted, based on the guidelines recommended by the Preferred Reporting Items for Systematic Reviews and Meta-Analysis (PRISMA) statement [15]. The review protocol was registered (PROSPERO registration number: CRD42020201751).

\section{Identification and selection of studies}

The search included the electronic databases PubMed, Medline, Embase, PEDro and Cochrane Library. Trials with the keywords "pelvic pain AND biofeedback" were extracted and considered for inclusion. No filters were used. No restrictions were placed on the year of publication. A systematic literature search was independently performed by two researchers (BW, MS) and disagreements in selection were resolved through discussion. The process was supervised by an experienced senior researcher (RC).

\section{Inclusion and exclusion criteria}

Table 1 presents the inclusion and exclusion criteria regarding study design, participants, interventions and outcome evaluation. Any quantitative study type of primary research (with the exception of case studies/case series less than 10 participants) was included to present a comprehensive overview of the current literature. This approach goes along with previous reviews $[5,13]$ which stated that including randomized controlled trials (RCTs) only was not feasible in reviewing physiotherapy interventions in patients with CPP. We considered males and females of all ages with either acute or chronic pelvic pain conditions as listed in the EAU guidelines [2], including both specific disease-associated pelvic pain and pelvic pain syndromes. Interventions were judged eligible if biofeedback was administered as a sole intervention or significant component of a multimodal or multidisciplinary intervention (including mechanical or electrical devices), as multidisciplinary management of CPP is considered optimal [13]. 


\section{Data collection and analysis}

For eligible papers, the following data were extracted: study characteristics (author and year of publication, country site, study design, sample sizes, drop-out rate, diagnoses investigated, author's conclusions), patient characteristics (sample characteristics, mean duration of symptoms, sex, mean age), intervention characteristics (interventions administered, time points of follow-up, biofeedback devices, training extent, intervention setting, adverse events) (Tables 2, 3 and 4). Primary outcome parameters were pain, overall symptom improvement and quality of life. Secondarily, physiological parameters were assessed. When certain data were not given in the respective studies the information was stated as "not available".

Outcome data were presented by means of the mean difference within a study group or between groups and their statistical significance (Tables 5, 6 and 7). Few studies provided effect sizes or the corresponding interval estimates (e.g. the confidence intervals) for the mean differences. These values were calculated by the authors if studies provided the relevant data to do so. The criteria for determining effect sizes according to Cohen [61] are listed in the legend of Tables 5 and 7.

\section{Data synthesis}

An attempt was made to bundle data for a meta-analysis; however, due to the substantial heterogeneity of study designs, patient characteristics, interventions and effect measures, a meta-analysis was not possible as results are considered unreliable when a small number of heterogeneous studies are assessed [70]. Rather, a narrative synthesis of study results was performed [71], and findings were juxtaposed in the respective tables to provide a comprehensive overview of the current literature.

\section{Quality assessment}

As trials differed in their study design, the McMaster Critical Review Form-Quantitative Studies [72, 73] was chosen for assessing the methodological quality of all studies included. This critical appraisal tool allows comparisons across different types of quantitative study designs due to its generic composition [5, $74,75]$. It comprises 15 items that evaluate method rigor and bias and has a guideline for completing the questionnaire that facilitates consistency in interpretation and application [72, 74]. In its original form, the tool did not provide a numerical summation. Based on previous reviews [5, 74, 75], for better comparability between included studies, a sum score of the respective subdomains was established. Each question is rated with either "yes" (1 point), "no", "not addressed" or "not applicable (N/A)" (0 points). In this arbitrary scoring system, higher scores indicate higher methodological quality, resulting in a possible total score of 14 points [5].
In addition, studies with an RCT design were evaluated using the Physiotherapy Evidence DatabasePEDro score, a valid and reliable tool for assessing the methodological quality and completeness of statistical reporting of randomized and quasi randomized controlled trials in physiotherapy [12, 76-81]. The tool evaluates internal validity and interpretability [82]. Eleven items are rated yes or no (1 or 0 points) according to whether the criterion is clearly satisfied in the study. A total PEDro score is achieved by adding the ratings of items 2-11 for a total score between 0 and 10. Higher scores indicate superior methodological quality. Studies with 9-10 points are considered excellent, 6-8 good, 4-5 fair and $<4$ poor quality [80].

\section{Results}

\section{Study selection}

A total of 651 studies published between 1978 and 29 July 2020 were found and screened for eligibility by title and abstract. After eliminating duplicates, 389 studies were rejected as non-includable, 83 studies were selected for full-text analysis and 37 articles corresponded to the inclusion criteria. Details on the systematic literature search and the selection process are presented in Fig. 1.

\section{Study characteristics}

\section{Quality assessment}

Table 8 shows the quality assessment using the Mc Master Critical Review Form-Quantitative Studies Tool for assessing the risk of bias of all studies included.

All studies but one were judged to have clearly stated the purpose of the study [38] and to have reviewed the relevant background literature [36]. The majority of the studies (29/37) gave enough detail on important sample characteristics. Only 10/37 studies stated how they arrived at the sample size. A minority of studies explicitly stated to have used reliable [21, $29,34,40,45,48,55]$ and valid $[21,25,26,34,35,37$, $40,47,53-55]$ outcome measures. For several tools, however, the psychometric properties are described in the literature. If at least one main outcome tool was used that is described in the literature, studies were marked with an asterisk. The majority of the studies (30/37) was judged to have described the intervention in detail. Where applicable/where addressed, most studies (13/14) were assessed to have avoided contamination through inadvertent treatment but not to have avoided co-interventions (17/23) as in many cases, subjects were taking medication during the study period (e.g. analgesics, laxatives in anorectal disorders). Most studies (34/37) reported results in terms of statistical significance, chose analysis methods appropriate for the study and the outcomes (32/35) and reported on drop-outs (26/37). All studies 
(37/37) were assessed to have discussed the relevance of the results to clinical practice and the majority of the studies (32/37) were judged to draw appropriate conclusions, given the study methods and results. The arbitrary sum score ranged between 5 and 13 (mean 9.2).

Table 9 shows the quality assessment of the 9 RCTs according to the PEDro scale [81], resulting in 2 studies of fair [26, 55] and 7 studies of good [16, 21, 42, $44,45,47,48$ ] quality. The mean PEDro score of these studies was 6 (range 5-8). All studies were randomized (9/9), analyzed the between-group difference (9/9), reported point estimate and variability (9/9) and had similar groups at baseline $(9 / 9)$. Some studies had a concealed allocation (4/9), 4 out of 9 studies reported adequate follow-up. The majority of the studies did not have blinded participants (8/9), blinded therapists (9/9) or blinded assessors (5/9). In 7 out of 9 studies all subjects for whom outcome measures were available received the treatment or control condition as allocated or, if this was not possible, data for at least one key outcome were analyzed by intention to treat [81].

Table 2 gives an overview of the characteristics of the included studies, additionally outlining study design, comparison characteristics and sample sizes.

\section{Participants}

A total of 2913 patients with pelvic pain conditions and 75 healthy subjects were included in 37 studies, of whom 2489 patients were assigned to groups receiving biofeedback. The other subjects received different treatment, no intervention or standard care (Table 4).

Table 3 (and Table 2) present the patient characteristics: 5 studies investigated patients with anorectal pain syndromes [16-20], 11 studies evaluated patients with constipation [21-31], 4 studied men with nonbacterial chronic prostatitis [32, 34-36], 12 investigated females with CPP (vulvar vestibulitis syndrome/ dyspareunia, pelvic floor dysfunction, dysmenorrhea, sexual dysfunction, or urethral syndrome) [37-45, 47, 48], 1 evaluated patients with low back pain [55] and 4 studied children with pelvic floor spasm [51], overactive bladder syndrome [52], dysfunctional voiding [53] or pubertal chronic prostatitis [54]. Overlapping diagnoses were common. The literature search only revealed chronic (no acute) pelvic pain conditions treated with biofeedback. The majority of the studies $(24 / 37)$ stated that a secondary cause of pelvic pain had been excluded [16-20, 22-28, 30, 32, 34-36, 38, 41, $42,48,52-54]$. One study enrolled patients with multiple sclerosis as an underlying disease [47], 6 studies $[18,20,23,25,27,30]$ indicated that the included subjects suffered from some kind of psychopathology (anxiety, depression, emotional trauma), 3 studies explicitly excluded patients with a psychopathologic disorder [16, 45, 48]. A total of 15 studies [18, 20, 21, 23-28, 32, 35, 38, 39, 42, 52] stated that conventional treatment including medication, changes in diet and interventions had failed prior to biofeedback.

Age ranged between 11 and 96 years in studies mainly enrolling adults. The mean age for trials involving children was 8.4 years [51-53] and 16.5 years for the study investigating adolescents [54].

\section{Intervention}

Table 4 presents an overview of the study intervention characteristics. 27 study protocols applied biofeedback only (together with counselling/education, pelvic floor exercises and home exercises, which are counted as part of the biofeedback intervention) [17, 18, 20-32, $34,36,38,39,42-45,48,51,53,54]$, others applied biofeedback as a multimodal treatment component (including psychological techniques [16, 19], electrotherapy [35, 37, 40, 41, 46, 47], medication [37], manual therapy [40] or guidelines-based care [51, 55]). Most studies evaluated outcome after the treatment, some (re)evaluated 2-3 months after the end of the treatment $[16,21,24,26,35,36,41-43,48]$, some had a long-term follow-up (6-mean 28 months) [16, $18,21-28,32,38,51]$.

Anorectal manometric systems and surface EMG techniques were the commonly applied anorectal physiological assessment tools in studies dealing with anorectal disorders. Male chronic pelvic pain syndromes used EMG-guided training [32, 34-36]. In urogenital phenotypes in children and adolescents, both urodynamics and perineal EMG were used. In female chronic pelvic pain syndromes, most studies used pelvic floor EMG to evaluate pelvic floor function. Three studies on patients with dysmenorrhea [43-45] aimed at increasing general relaxation by using heart rate variability training, skin temperature training and EMG of the frontalis and lower abdominal muscles.

Overall, the biofeedback training extent was largely heterogeneous, 2-30 sessions were administered, lasting between 10 and $60 \mathrm{~min}$, for up to 6 months. Most designs applied biofeedback weekly, less often sessions were scheduled twice or three times a week or once every 2 weeks. Biofeedback in a home-based setting was applied daily in 3 studies on gynecological disorders [38, 39, 45]. Treating anorectal disorders, four large trials by Chiarioni et al. [16, 21, 28] and Ba-Bai-Ke-Re et al. [26] proved 5 weekly biofeedback sessions of 30min to be successful (Table 4).

Of the studies 11 reported that no biofeedback-related side effects had occurred [16-19, 21, 25, 29, 30, $35,48,52]$ and 1 study noted a transient skin irritation related to the use of a tape [55].

\section{Outcome}

Primarily evaluated outcomes: pain intensity, overall symptom improvement, quality of life Heterogeneous assessment methods were used to evaluate primary outcome measures within a certain phenotype (Tables 5 and 7). Pain was assessed using either visual 
Table 3 Patient characteristics

\begin{tabular}{|c|c|c|c|c|c|}
\hline \multirow[t]{2}{*}{ Study } & \multirow{2}{*}{$\begin{array}{l}\text { Subgroups according to physiological testing (if } \\
\text { applicable) }\end{array}$} & \multirow{2}{*}{$\begin{array}{l}\text { Mean symptom dura- } \\
\text { tion } \pm \text { SD (range) }\end{array}$} & \multicolumn{2}{|l|}{ Sex $\%$} & \multirow{2}{*}{$\begin{array}{l}\text { Mean age IG in } \\
\text { years } \pm S D \text { (range) }\end{array}$} \\
\hline & & & Male & Female & \\
\hline \multicolumn{6}{|c|}{ Anorectal pain syndrome } \\
\hline $\begin{array}{l}\text { Chiarioni et al. } \\
2010[16]\end{array}$ & n.a. & $\begin{array}{l}17.1 \pm 4.3 \text { months ("high } \\
\text { likely" LAS), } 18.6 \pm 4.8 \\
\text { ("possible" LAS) }\end{array}$ & $\begin{array}{l}\text { 19\% ("high likely" } \\
\text { LAS), 33\% (“possi- } \\
\text { ble" LAS) }\end{array}$ & $48 \%$ & $\begin{array}{l}41.0 \pm 10.0 \text { in "high } \\
\text { likely" LAS, } 41.4 \pm 10.3 \\
\text { in "possible" LAS }\end{array}$ \\
\hline $\begin{array}{l}\text { Heah et al. } \\
1997[17]\end{array}$ & n.a. & $32.5 \pm 6.7$ months & $56.3 \%^{b}$ & $43.6 \%^{b}$ & $50.5(39-66)$ \\
\hline $\begin{array}{l}\text { Ger et al. } 1993 \\
\text { [18] }\end{array}$ & n.a. & 54 (2-228) months & $42.9 \%^{b}$ & $57.1 \%^{b}$ & 71 (n.a.) $^{b}$ \\
\hline $\begin{array}{l}\text { Gilliland et al. } \\
\text { 1997a [19] }\end{array}$ & $\begin{array}{l}\text { High resting pressures (manometry): } n=5 / 14 \text {, non- } \\
\text { relaxation or paradoxical PF contraction: } n=7 / 14 \text {, } \\
\text { abnormal cinedefecography: } n=14 / 14\end{array}$ & 36 (3-lifelong) months & $36.0 \%^{b}$ & $64.0 \%^{b}$ & $68(12-96)^{b}$ \\
\hline $\begin{array}{l}\text { Grimaud et al. } \\
1991[20]\end{array}$ & n.a. & $8 \pm 6$ (3-72) months & $66.7 \%^{b}$ & $33.3 \%^{b}$ & $54 \pm 3(24-66)^{b}$ \\
\hline \multicolumn{6}{|c|}{ Constipation, dyssynergic defecation } \\
\hline $\begin{array}{l}\text { Chiarioni et al. } \\
2006 \text { [21] }\end{array}$ & Slow transit constipation was excluded & $>12$ months & $5.6 \%$ & $94.4^{b}$ & $33.3 \pm 1.5^{b}$ \\
\hline $\begin{array}{l}\text { Koutsomanis } \\
\text { et al. } 1994 \\
\text { [22] }\end{array}$ & $\begin{array}{l}\text { Slow transit: } n=2 / 20 \text {, slow transit + pelvic incoor- } \\
\text { dination: } n=11 / 20 \text {, pelvic incoordination: } n=7 / 20\end{array}$ & n.a. & $90.0 \%{ }^{b}$ & $10.0 \%^{b}$ & $34(18-53)^{b}$ \\
\hline $\begin{array}{l}\text { Chiotakakou- } \\
\text { Faliakou et al. } \\
1998[23]\end{array}$ & $\begin{array}{l}\text { Slow transit: } n=18 / 100 \text {, slow transit + paradoxcial } \\
\text { PF contraction: } n=29 / 100 \text {, normal transit: } \\
n=11 / 100 \text {, normal transit + paradoxical PF con- } \\
\text { traction: } n=15 / 100\end{array}$ & $\begin{array}{l}\text { n.a., median age of onset: } \\
21(0-70)\end{array}$ & $13.0 \%^{b}$ & $87.0 \%^{b}$ & $40(10-79)^{b}$ \\
\hline $\begin{array}{l}\text { Battaglia et al. } \\
2004[24]\end{array}$ & PF dyssynergia, $n=14 / 24$, slow transit: $n=10 / 24$ & $>12$ months & $16.7 \%^{b}$ & $83.3 \%^{b}$ & n.a., $(27-54)^{b}$ \\
\hline $\begin{array}{l}\text { Wang et al. } \\
2003 \text { [25] }\end{array}$ & $\begin{array}{l}\text { Slow transit: } n=8 / 50 \text {, anorectic outlet obstruction: } \\
n=36 / 50 \text {, both: } n=6 / 50\end{array}$ & $55.2(30-360)$ months & $28.0 \%^{b}$ & $72.0 \%^{\mathrm{b}}$ & $52.6(16-71)^{b}$ \\
\hline $\begin{array}{l}\text { Ba-Bai-Ke- } \\
\text { Re et al. } 2014 \\
{[26]}\end{array}$ & n.a. & 42 months & n.a. & n.a. & 54 (n.a.) \\
\hline $\begin{array}{l}\text { Roy et al. } \\
2000 \text { [27] }\end{array}$ & $\begin{array}{l}\text { Rectal prolapse: } n=12 / 78 \text {, rectocele: } n=22 / 78 \text {, } \\
\text { slow transit: } n=53 / 78 \text {, paradoxical PF contrac- } \\
\text { tion: } n=40 / 78\end{array}$ & Several years & $0 \%^{\mathrm{b}}$ & $100 \%{ }^{b}$ & n.a. $(24-75)^{b}$ \\
\hline $\begin{array}{l}\text { Chiarioni et al. } \\
2005[28]\end{array}$ & n.a. & $168(24-480)$ months & $05.8 \%{ }^{\mathrm{b}}$ & $94.2 \%$ b & $34.9 \pm 10.2(23-63)^{b}$ \\
\hline $\begin{array}{l}\text { Zhu et al. } \\
2011 \text { [29] }\end{array}$ & n.a. & n.a. & $38.9 \%^{b}$ & $61.1 \%^{b}$ & $46.4(21-65)^{b}$ \\
\hline $\begin{array}{l}\text { Gilliland et al. } \\
1997 b[30]\end{array}$ & $\begin{array}{l}\text { Fixed or dynamic descent: } n=100 / 194 \text {, rectocele: } \\
n=32 / 194 \text {, intussusception: } n=15 / 194\end{array}$ & 168 (2-lifelong) months & $30.4 \%{ }^{b}$ & $69.6 \%{ }^{b}$ & $71(11-96)^{b}$ \\
\hline $\begin{array}{l}\text { Parker et al. } \\
2019 \text { [31] }\end{array}$ & $\begin{array}{l}\text { Chronic constipation + dyssynergic defecation } \\
n=53 / 130 \text {, without: } n=3 / 130\end{array}$ & n.a. & $20.8 \%{ }^{b}$ & $79.2 \%^{\mathrm{b}}$ & $57.5 \pm 16.4^{b}$ \\
\hline \multicolumn{6}{|c|}{ Male chronic pelvic pain syndrome, urological chronic pelvic pain syndrome } \\
\hline $\begin{array}{l}\text { Clemens et al. } \\
2000 \text { [32] }\end{array}$ & n.a. & n.a. & $100 \%^{b}$ & $0 \%^{b}$ & $38(18-67)^{b}$ \\
\hline $\begin{array}{l}\text { Cornel et al. } \\
2005[34]\end{array}$ & $\begin{array}{l}\text { Detrusor instability: } n=5 / 19 \text {, diminished bladder } \\
\text { capacity: } n=4 / 19 \text {, dysfunctional voiding of blad- } \\
\text { der (pseudodyssynergia): } n=6 / 19 \text {, cystometric } \\
\text { abnormalities + pseudodyssynergia } n=3 / 19\end{array}$ & $\geq 3$ months & $100 \%^{b}$ & $0 \%^{b}$ & $45(23-70)^{b}$ \\
\hline $\begin{array}{l}\text { Yang et al. } \\
2017 \text { [35] }\end{array}$ & n.a. & 30.4 (6-144) months & $100 \%^{b}$ & $0 \%^{b}$ & $43.4(24-68)^{b}$ \\
\hline $\begin{array}{l}\text { He et al. } 2010 \\
{[36]}\end{array}$ & n.a. & $>3$ months & $100 \%$ b & $0 \%$ & n.a. \\
\hline
\end{tabular}


review article

\begin{tabular}{|c|c|c|c|c|c|}
\hline \multirow[t]{2}{*}{ Study } & \multirow{2}{*}{$\begin{array}{l}\text { Subgroups according to physiological testing (if } \\
\text { applicable) }\end{array}$} & \multirow{2}{*}{$\begin{array}{l}\text { Mean symptom dura- } \\
\text { tion } \pm \text { SD (range) }\end{array}$} & \multicolumn{2}{|l|}{ Sex $\%$} & \multirow{2}{*}{$\begin{array}{l}\text { Mean age IG in } \\
\text { years } \pm S D \text { (range) }\end{array}$} \\
\hline & & & Male & Female & \\
\hline \multicolumn{6}{|c|}{ Female chronic pelvic pain } \\
\hline $\begin{array}{l}\text { Schmitt et al. } \\
2017[37]\end{array}$ & n.a. & n.a. & $0 \%$ & $100 \%$ & $\begin{array}{l}52.2 \pm 15.4 \text { in } n=29 \text { with } \\
\text { pelvic pain }\end{array}$ \\
\hline $\begin{array}{l}\text { Glazer et al. } \\
1995[38]\end{array}$ & n.a. & 40.8 (24-72) months & $0 \%$ & $100 \%$ & $31.5(21-45)$ \\
\hline $\begin{array}{l}\text { McKay et al. } \\
2001[39]\end{array}$ & n.a. & $44.4(24-60)$ months & $0 \%$ & $100 \%$ & $35(25-48)$ \\
\hline $\begin{array}{l}\text { Gentilcore- } \\
\text { Saulnier et al. } \\
2010[40]\end{array}$ & n.a. & $48 \pm 12$ months & $0 \%$ & $100 \%$ & $22 \pm 2$ \\
\hline $\begin{array}{l}\text { Bendana et al. } \\
2009 \text { [41] }\end{array}$ & n.a. & n.a. & $0 \%$ & $100 \%$ & $45.0 \pm 17,(19-76)$ \\
\hline $\begin{array}{l}\text { Philips et al. } \\
1992[42]\end{array}$ & n.a. & 57 (4-240) months & $0 \%^{\mathrm{b}}$ & $100 \%^{b}$ & $25.7 \pm 4.7^{b}$ \\
\hline $\begin{array}{l}\text { Hart et al. } \\
1981[43]\end{array}$ & n.a. & n.a. & $0 \%$ & $100 \%$ & $26 \pm 6.2$ \\
\hline $\begin{array}{l}\text { Bennink et al. } \\
1982[44]\end{array}$ & n.a. & n.a. & $0 \%^{a}$ & $100 \%^{a}$ & 19.2 (n.a.) ${ }^{\mathrm{a}}$ \\
\hline $\begin{array}{l}\text { Vagedes et al. } \\
2019 \text { [45] }\end{array}$ & n.a. & $>1$ year & $0 \%^{a}$ & $100 \%^{a}$ & $29.7 \pm 8.0^{\mathrm{a}}$ \\
\hline $\begin{array}{l}\text { Starr et al. } \\
2013[46]\end{array}$ & n.a. & n.a. & $0 \%^{\mathrm{b}}$ & $100 \%^{b}$ & $51(18-95)^{b}$ \\
\hline $\begin{array}{l}\text { Lúcio et al. } \\
2014 \text { [47] }\end{array}$ & n.a. & $4.1(0.7-10)$ months & $0 \%^{b}$ & $100 \%^{b}$ & $\begin{array}{l}44.5(36-51)^{b} \text { in interven- } \\
\text { tion group } 1\end{array}$ \\
\hline $\begin{array}{l}\text { Aalaie et al. } \\
2020 \text { [48] }\end{array}$ & n.a. & n.a. & $0 \%$ & $100 \%$ & $50.7 \pm 6.1$ \\
\hline \multicolumn{6}{|c|}{ Chronic pelvic pain in children } \\
\hline $\begin{array}{l}\text { Hoebeke et al. } \\
2004 \text { [51] }\end{array}$ & n.a. & 3 months & $9.5 \%$ & $90.5 \%$ & 8.3 (n.a.) \\
\hline $\begin{array}{l}\text { Ebiloglu et al. } \\
2016[52]\end{array}$ & $\begin{array}{l}\text { Overactive bladder syndrome + dysfunctional void- } \\
\text { ing: } n=107 / 136 \text {, overactive bladder syndrome } \\
\text { only: } n=29 / 136\end{array}$ & n.a. & $29.0 \%{ }^{b}$ & $71.0 \%{ }^{\mathrm{b}}$ & $8.11(5-14)^{b}$ \\
\hline $\begin{array}{l}\text { Ergin et al. } \\
2016[53]\end{array}$ & n.a. & n.a. & $23.1 \%^{b}$ & $76.9 \%^{b}$ & $8.84 \pm 2.54^{b}$ \\
\hline $\begin{array}{l}\text { Li et al. } 2006 \\
{[54]}\end{array}$ & n.a. & $>3$ months & $100 \%^{\mathrm{b}}$ & $0 \%$ & $16.5 \pm 1.1(15-18)^{b}$ \\
\hline \multicolumn{6}{|c|}{ Musculoskeletal, low back pain, myofascial pain } \\
\hline $\begin{array}{l}\text { Kent et al. } \\
2015[55]\end{array}$ & n.a. & $13(4.25-13)$ months & $48.0^{\mathrm{b}}$ & $52.0 \%{ }^{b}$ & $39 \pm 12^{b}$ \\
\hline
\end{tabular}

analog scale (VAS) or numeric rating scale (NRS) [16, $17,32,35,37-40,45,55,60$ ] or subdomains of relevant questionnaires [29, 34-36, 47, 48, 54]. In terms of overall symptom improvement, several studies used symptom scores [26, 32, 34-36, 41, 43, 44, 47, 48, 52-54]. Apart from using standardized questionnaires, many studies reported the success rate, given as the number or percentage of patients who stated subjective pain or symptom improvement. Definitions regarding the extent of symptom improvement differed between studies (Tables 5, 6 and 7).

Quality of life was only assessed in 9 studies [26, 29, 34-36, 40, 41, 45, 54], applying questionnaires, subdomains of validated symptom scores or impact on quality of life on a VAS or NRS scale [60].
Outcome tools together with references of the respective questionnaires are outlined in Tables 5 and 7.

Secondarily evaluated outcomes: physiological parameters Pelvic floor function was assessed using manometric devices, urodynamic devices as well as surface EMG techniques and digital examination. One study observed general relaxation through heart rate variability measures [45].

\section{Effect of biofeedback interventions on pain, overall symptoms}

Table 5 presents the effect of biofeedback-assisted interventions on pain and overall symptom improve- 
Table 4 Intervention characteristics

\begin{tabular}{|c|c|c|c|c|c|}
\hline Study & $\begin{array}{l}\text { Interventions performed in } \\
\text { longitudinal study arm }\end{array}$ & Time points of follow-up & BFB device details & $\begin{array}{l}\text { BFB treatment details: number } \\
\text { of sessions, duration per session } \\
\text { (min/, frequency) (times/week), } \\
\text { duration of whole intervention } \\
\text { (weeks, if available) }\end{array}$ & $\begin{array}{l}\text { Setting of BFB inter- } \\
\text { vention (clinical/home- } \\
\text { based), home exer- } \\
\text { cise (=HE) encouraged } \\
\text { (yes/not addressed) }\end{array}$ \\
\hline \multicolumn{6}{|c|}{ Anorectal pain syndrome } \\
\hline \multirow[t]{3}{*}{$\begin{array}{l}\text { Chiarioni et } \\
\text { al.2010 [16] }\end{array}$} & $\begin{array}{l}\text { IG 1: BFB + psychological } \\
\text { counselling ( } 5 \text { sessions } \\
\text { BFB }+4 \text { sessions psycho- } \\
\text { logical counselling) }(n=52)\end{array}$ & $\begin{array}{l}\text { Baseline, 1, 3, } 6 \text {, } \\
12 \text { months }\end{array}$ & Anal sEMG probe & 5 sessions, $30 \mathrm{~min}, 1 \times /$ week & Clinical \\
\hline & $\begin{array}{l}\text { IG 2: EGS }(9 \text { ses- } \\
\text { sions, } 30-45 / m i n, 3 \times / \\
\text { week) }+4 \text { sessions psycho- } \\
\text { logical counselling }(n=52)\end{array}$ & $\begin{array}{l}\text { Baseline, 1, 3, 6, } \\
12 \text { months }\end{array}$ & - & - & - \\
\hline & $\begin{array}{l}\text { IG 3: massage of leva- } \\
\text { tor ani muscle }(9 \text { ses- } \\
\text { sions, } 30-45 \text { min, } 3 \times \text { / } \\
\text { week }+4 \text { sessions psycho- } \\
\text { logical counselling }(n=53)\end{array}$ & $\begin{array}{l}\text { Baseline, 1, 3, } 6 \text {, } \\
12 \text { months }\end{array}$ & - & - & - \\
\hline $\begin{array}{l}\text { Heah et al. } \\
1997[17]\end{array}$ & BFB & $\begin{array}{l}\text { Baseline }-2 \text { weeks af- } \\
\text { ter treatment, mean } \mathrm{f} / \mathrm{u} \\
12.8 \pm 2.6 \text { months }\end{array}$ & $\begin{array}{l}\text { Anorectal manometry } \\
\text { (rectal balloon) }\end{array}$ & 4 sessions, $60 \mathrm{~min}, 1 \times /$ week & Clinical (HE: yes) \\
\hline \multirow[t]{3}{*}{$\begin{array}{l}\text { Ger et al. } \\
1993[18]\end{array}$} & $\begin{array}{l}\text { IG 1: EGS ( } 3 \text { sessions, } \\
30-60 \text { min, } 7-10 \text { days) } \\
\text { (some had failed BFB, } \\
\text { epitural caudal block ear- } \\
\text { lier) }\end{array}$ & $\begin{array}{l}\text { Baseline-mean f/u } 15 \\
\text { [2-36] months after treat- } \\
\text { ment }\end{array}$ & - & - & - \\
\hline & $\begin{array}{l}\text { IG 2: BFB ( } 50 \% \text { had failed } \\
\text { EGS earlier) }\end{array}$ & Same as IG1 & Anal sEMG probe & $\begin{array}{l}\geq 6 \text { sessions, } 30-60 \mathrm{~min}, 1 \times / \\
\text { week }\end{array}$ & Clinical (HE: yes) \\
\hline & $\begin{array}{l}\text { IG 3: epidural steroid } \\
\text { caudal block (some had } \\
\text { failed other modalities } \\
\text { earlier) }\end{array}$ & Same as IG1 & - & - & - \\
\hline $\begin{array}{l}\text { Gilliland et al. } \\
\text { 1997a [19] }\end{array}$ & $\begin{array}{l}\text { BFB including education, } \\
\text { stress management and } \\
\text { cognitive-behavioral psy- } \\
\text { chotherapy techniques }\end{array}$ & $\begin{array}{l}\text { Baseline-after treatment } \\
\text { (time-points varied) }\end{array}$ & Anal sEMG probe & $\begin{array}{l}\text { 2-18 sessions (until improve- } \\
\text { ment/persistent failure/self- } \\
\text { discharge), } 60 \text { min; frequency n.a. }\end{array}$ & Clinical (HE: yes) \\
\hline $\begin{array}{l}\text { Grimaud et al. } \\
1991[20]\end{array}$ & BFB + education & $\begin{array}{l}\text { Baseline-after treatment, } \\
\text { long term f/u after } 16 \pm 1 \\
\text { [10-24] months }\end{array}$ & Anorectal manometry & $\begin{array}{l}5-13 \text { sessions, } 30 \mathrm{~min}, 1 \times / \\
\text { week until pain disappearance } \\
(8 \pm 1 \text { [5-13] weeks), reeducation } \\
\text { sessions over } 6 \text { months }\end{array}$ & Clinical (HE: yes) \\
\hline \multicolumn{6}{|c|}{ Constipation, dyssynergic defecation } \\
\hline \multirow[t]{2}{*}{$\begin{array}{l}\text { Chiarioni } \\
2006[21]\end{array}$} & IG1: BFB & $\begin{array}{l}\text { Baseline, } 6,12,24 \text { months } \\
\text { after starting treatment }\end{array}$ & Anal sEMG probe & 5 sessions, $30 \mathrm{~min}, 1 \times /$ week & $\begin{array}{l}\text { Clinical, laxatives at } \\
\text { home }\end{array}$ \\
\hline & $\begin{array}{l}\text { IG2: laxatives (polyethy- } \\
\text { lene glycol } 1-2 \text { packets } \\
\text { daily + counselling with } \\
\text { physician) }\end{array}$ & Same as IG1 & - & - & - \\
\hline $\begin{array}{l}\text { Koutsomanis } \\
\text { et al. } 1994 \\
\text { [22] }\end{array}$ & BFB & $\begin{array}{l}\text { Baseline, after treatment, } \\
6 \text { weeks after starting } \\
\text { treatment, } 6-12 \text { months } \\
\text { after 6-week-f/u }\end{array}$ & $\begin{array}{l}\text { sEMG skin electrodes } \\
\text { close to anal verge (ex- } \\
\text { ternal anal sphincter), } \\
\text { visual + acoustic feedback }\end{array}$ & $\begin{array}{l}\text { Mean } 4 \text { [2-6] sessions (until im- } \\
\text { provement or persistent failure), } \\
30-45 \mathrm{~min}, 1 \times / \text { week }\end{array}$ & Clinical (HE: n.a.) \\
\hline $\begin{array}{l}\text { Chiotakakou- } \\
\text { Faliakou et al. } \\
1998 \text { [23] }\end{array}$ & $\begin{array}{l}\text { BFB + education, balloon } \\
\text { defecation training }\end{array}$ & $\begin{array}{l}\text { Baseline, after treatment, } \\
\text { long term f/u (mean } 23.4 \\
\text { [12-44]) months after } \\
\text { treatment }\end{array}$ & $\begin{array}{l}\text { sEMG skin electrodes } \\
\text { close to anal verge (exter- } \\
\text { nal anal sphincter), visual } \\
\text { feedback }\end{array}$ & $\begin{array}{l}\text { Mean } 4 \text { [1-10] sessions, duration } \\
\text { n.a., } 1 \times / 1-2 \text { weeks }\end{array}$ & Clinical (HE: yes) \\
\hline $\begin{array}{l}\text { Battaglia et al. } \\
2004 \text { [24] }\end{array}$ & $\begin{array}{l}\mathrm{BFB}+\text { balloon defecation } \\
\text { training }\end{array}$ & $\begin{array}{l}\text { Baseline, } 3 \text { months, } 1 \text { year } \\
\text { after treatment }\end{array}$ & Anal sEMG plug & $\begin{array}{l}8 \text { sessions, duration n.a., } 2 \times 1 \\
\text { week, over } 4 \text { weeks }\end{array}$ & Clinical (HE: yes) \\
\hline
\end{tabular}


Table 4 (Continued)

\begin{tabular}{|c|c|c|c|c|c|}
\hline Study & $\begin{array}{l}\text { Interventions performed in } \\
\text { longitudinal study arm }\end{array}$ & Time points of follow-up & BFB device details & $\begin{array}{l}\text { BFB treatment details: number } \\
\text { of sessions, duration per session } \\
\text { (min/, frequency) (times/week), } \\
\text { duration of whole intervention } \\
\text { (weeks, if available) }\end{array}$ & $\begin{array}{l}\text { Setting of BFB inter- } \\
\text { vention (clinical/home- } \\
\text { based), home exer- } \\
\text { cise (=HE) encouraged } \\
\text { (yes/not addressed) }\end{array}$ \\
\hline $\begin{array}{l}\text { Wang et al. } \\
2003 \text { [25] }\end{array}$ & $\begin{array}{l}\text { BFB: EMG vs. manometry } \\
\text { based BFB }\end{array}$ & $\begin{array}{l}\text { Baseline, after treatment, } \\
\text { long term f/u (mean18 } \\
\text { [12-28] months after } \\
\text { treatment) }\end{array}$ & $\begin{array}{l}\text { EMG based BFB: sur- } \\
\text { face sEMG electrodes } \\
\text { (anal sphincter), audi- } \\
\text { tory + visual feedback; } \\
\text { manometry based BFB: } \\
\text { visual BFB }\end{array}$ & 5 sessions, $30 \mathrm{~min}, 1 \times /$ week & Clinical (HE: yes) \\
\hline \multirow{2}{*}{$\begin{array}{l}\text { Ba-Bai-Ke- } \\
\text { Re et al. } 2014 \\
{[26]}\end{array}$} & IG1: BFB & $\begin{array}{l}\text { Baseline, } 1,3,6 \text { months } \\
\text { after treatment }\end{array}$ & Anorectal manometry & $\begin{array}{l}4-5 \text { sessions, duration n.a., } \\
1 / 1-2 \text { week }\end{array}$ & Clinical (HE: yes) \\
\hline & $\begin{array}{l}\text { IG2: laxatives (polyethy- } \\
\text { lene glycol, } 17 \mathrm{~g} 3 \times / \text { day, } \\
2 \text { weeks) }\end{array}$ & - & - & - & - \\
\hline $\begin{array}{l}\text { Roy et al. } \\
2000 \text { [27] }\end{array}$ & BFB & $\begin{array}{l}\text { Baseline, after treatment, } \\
\text { long-term f/u (mean } 28 \\
{[12-44] \text { months after }} \\
\text { treatment) }\end{array}$ & $\begin{array}{l}\text { sEMG skin electrodes } \\
\text { close to anal verge (exter- } \\
\text { nal anal sphincter), visual } \\
\text { feedback }\end{array}$ & $\begin{array}{l}4-5 \text { sessions, duration n.a. } 1 / 1-2 \\
\text { week }\end{array}$ & Clinical (HE: n.a.) \\
\hline $\begin{array}{l}\text { Chiarioni et } \\
\text { al. } 2005[28]\end{array}$ & $\begin{array}{l}\mathrm{BFB}+\text { balloon defecation } \\
\text { training }\end{array}$ & $\begin{array}{l}\text { Baseline, } 1,6,12, \\
24 \text { months after treatment }\end{array}$ & $\begin{array}{l}\text { Anal sEMG plug, visual } \\
\text { feedback }\end{array}$ & 5 sessions, $30-45 \mathrm{~min}, 1 \times /$ week & Cinical (HE: n.a.) \\
\hline $\begin{array}{l}\text { Zhu et al. } \\
2011 \text { [29] }\end{array}$ & BFB & $\begin{array}{l}\text { Baseline-after treatment } \\
\text { (n.a.) }\end{array}$ & $\begin{array}{l}\text { Water-perfused intra-anal } \\
\text { instrument, visual + verbal } \\
\text { feedback }\end{array}$ & $\begin{array}{l}6-10 \text { sessions, } 30-60 \mathrm{~min} \text {, fre- } \\
\text { quency n.a., over 4-8 weeks }\end{array}$ & Clinical (HE: yes) \\
\hline $\begin{array}{l}\text { Gilliland et al. } \\
\text { 1997b [30] }\end{array}$ & $\begin{array}{l}\text { BFB (+education, stress } \\
\text { management, lifestyle } \\
\text { modification) }\end{array}$ & $\begin{array}{l}\text { Baseline-after treatment } \\
\text { (n.a.) }\end{array}$ & Anal sEMG probe & $\begin{array}{l}2->30 \text { sessions (until symptom } \\
\text { resolution/control over PF mus- } \\
\text { cles in EMG/self-discharge: mean } \\
\text { self-discharged: } 5 \text {, finished: } 11 \text { ), } \\
60 \text { min, further data n.a }\end{array}$ & Clinical (HE: yes) \\
\hline $\begin{array}{l}\text { Parker et al. } \\
2019 \text { [31] }\end{array}$ & $\begin{array}{l}\text { BFB (+education, exercise } \\
\text { instructions, diet) }\end{array}$ & $\begin{array}{l}\text { Baseline-after treatment } \\
\text { (n.a.) }\end{array}$ & $\begin{array}{l}\text { Anorectal manometry, } \\
\text { visual feedback }\end{array}$ & $\begin{array}{l}\text { Mean } 2.9 \text { [2-3] sessions, further } \\
\text { data n.a. }\end{array}$ & Clinical (HE: yes) \\
\hline \multicolumn{6}{|c|}{ Male chronic pelvic pain syndrome, Urological Chronic Pelvic Pain Syndrome } \\
\hline $\begin{array}{l}\text { Clemens et } \\
\text { al. } 2000 \text { [32] }\end{array}$ & $\begin{array}{l}\text { BFB (PF reeduca- } \\
\text { tion + bladder training) }\end{array}$ & $\begin{array}{l}\text { Baseline-mean } 5.8 \\
{[1.6-14.8] \text { months after }} \\
\text { treatment }\end{array}$ & sEMG electrodes & $\leq 6$ sessions, $60 \mathrm{~min}, 1 \times / 2$ week & Clinical (HE: yes) \\
\hline $\begin{array}{l}\text { Cornel et al. } \\
2005 \text { [34] }\end{array}$ & BFB & $\begin{array}{l}\text { Baseline-after treatment } \\
\text { (n.a.) }\end{array}$ & Anal sEMG probe & $\begin{array}{l}6-8 \text { sessions, } 1 \times / \text { week, later } \\
1 \times / 2-4 \text { weeks, duration n.a. }\end{array}$ & Clinical (HE: n.a.) \\
\hline \multirow[t]{2}{*}{$\begin{array}{l}\text { Yang et al. } \\
2017 \text { [35] }\end{array}$} & IG 1: EGS + BFB & $\begin{array}{l}\text { Baseline }-12 \text { weeks after } \\
\text { treatment }\end{array}$ & Anal sEMG probe & $\begin{array}{l}8 \text { sessions, } 45 \mathrm{~min} \text { ( } 15 \mathrm{~min} \text { BFB, } \\
30 \mathrm{~min} \text { EGS), } 1-2 \times / \text { week, over } \\
6 \text { weeks }\end{array}$ & Clinical (HE: n.a.) \\
\hline & $\begin{array}{l}\text { IG 2: electomagnetic stimu- } \\
\text { lation ( } 18 \text { sessions, } 30 \mathrm{~min} \text {, } \\
3 \times / \text { week, } 6 \text { weeks) }\end{array}$ & $\begin{array}{l}\text { Baseline-12 weeks after } \\
\text { treatment }\end{array}$ & - & - & - \\
\hline $\begin{array}{l}\text { He et al. } \\
2010[36]\end{array}$ & BFB & $\begin{array}{l}\text { Baseline-10 weeks after } \\
\text { treatment }\end{array}$ & Anal sEMG probe & $\begin{array}{l}\text { No. sessions n.a., } 30 \mathrm{~min}, 2-3 \times 1 \\
\text { week, over several weeks }\end{array}$ & Clinical (HE: n.a.) \\
\hline \multicolumn{6}{|c|}{ Female chronic pelvic pain } \\
\hline $\begin{array}{l}\text { Schmitt et } \\
\text { al. } 2017 \text { [37] }\end{array}$ & $\begin{array}{l}\text { BFB + vaginal } \\
\text { EGS + behavioral modifi- } \\
\text { cation + pharmacologic } \\
\text { therapies for urinary and } \\
\text { defecatory management }\end{array}$ & $\begin{array}{l}\text { Baseline, after 1st, 3rd, } \\
\text { final treatment session }\end{array}$ & $\begin{array}{l}\text { SEMG skin electrodes } \\
\text { (abdominals), vaginal/ } \\
\text { rectal sEMG probe }\end{array}$ & $\begin{array}{l}4-7 \text { sessions (until } \geq 80 \% \text { im- } \\
\text { provement), BFB }+30 \text { min vaginal } \\
\text { EGS, } 1 \times / 2 \text { week }\end{array}$ & Clinical (HE: yes) \\
\hline $\begin{array}{l}\text { Glazer et al. } \\
1995 \text { [38] }\end{array}$ & $\mathrm{BFB}$ & $\begin{array}{l}\text { Baseline, f/u at } 6 \text { clini- } \\
\text { cal evaluation appoint- } \\
\text { ments }+6 \text { months after } 6 \text { th } \\
\text { reevaluation }\end{array}$ & $\begin{array}{l}\text { SEMG portable vagi- } \\
\text { nal probe, visual } \\
\text { feedback }\end{array}$ & $\begin{array}{l}20 \mathrm{~min}, 2 \times / \text { day, } 7 \times / \text { week, after } \\
6 \text { evaluations: exercises con- } \\
\text { tinued without } B F B \geq 3 \text { months }\end{array}$ & $\begin{array}{l}\text { Home-based, } \\
6 \times \text { clinical f/u }\end{array}$ \\
\hline $\begin{array}{l}\text { McKay et al. } \\
2001 \text { [39] }\end{array}$ & BFB & $\begin{array}{l}\text { Baseline, f/u every } \\
4 \text { weeks }\end{array}$ & $\begin{array}{l}\text { sEMG portable vaginal } \\
\text { probe, visual feedback }\end{array}$ & $\begin{array}{l}\text { No. sessions n.a., duration n.a., } \\
60 \text { repetitions, } 2 \times / \text { day, } 7 \times / \text { week, } \\
\text { up to } 11 \text { months }\end{array}$ & $\begin{array}{l}\text { Home-based, } \\
1 \times / 4 \text { weeks clinical } \\
\text { f/u }\end{array}$ \\
\hline $\begin{array}{l}\text { Gentilcore- } \\
\text { Saulnier et al. } \\
2010 \text { [40] }\end{array}$ & $\begin{array}{l}\text { BFB + education, manual } \\
\text { therapy, EGS, dilator inser- } \\
\text { tion }\end{array}$ & $\begin{array}{l}\text { Baseline-after treatment } \\
\text { (n.a.) }\end{array}$ & $\begin{array}{l}\text { sEMG vaginal probe (deep } \\
\text { PF), sEMG electrodes } \\
\text { (superficial PF) }\end{array}$ & $\begin{array}{l}8 \text { sessions, } 60-75 \text { min overall } \\
\text { (10-15 min BFB), frequency n.a, } \\
\text { over } 12 \pm 3 \text { weeks }\end{array}$ & Clinical (HE: yes) \\
\hline $\begin{array}{l}\text { Bendana et } \\
\text { al. } 2009 \text { [41] }\end{array}$ & $\begin{array}{l}\text { BFB + education, vaginal } \\
\text { EGS }\end{array}$ & $\begin{array}{l}\text { Baseline, after treatment, } \\
3 \text { months after treatment }\end{array}$ & sEMG vaginal probe & $\begin{array}{l}6 \text { sessions, } 60 \mathrm{~min}(10 \mathrm{~min} B F B \text {, } \\
20 \mathrm{~min} \text { EGS), } 1 \times / \text { week }\end{array}$ & Clinical (HE: n.a.) \\
\hline
\end{tabular}


Table 4 (Continued)

\begin{tabular}{|c|c|c|c|c|c|}
\hline Study & $\begin{array}{l}\text { Interventions performed in } \\
\text { longitudinal study arm }\end{array}$ & Time points of follow-up & BFB device details & $\begin{array}{l}\text { BFB treatment details: number } \\
\text { of sessions, duration per session } \\
\text { ( } \mathrm{min} / \text {, frequency) (times/week), } \\
\text { duration of whole intervention } \\
\text { (weeks, if available) }\end{array}$ & $\begin{array}{l}\text { Setting of BFB inter- } \\
\text { vention (clinical/home- } \\
\text { based), home exer- } \\
\text { cise (=HE) encouraged } \\
\text { (yes/not addressed) }\end{array}$ \\
\hline \multirow[t]{3}{*}{$\begin{array}{l}\text { Philips et al. } \\
1992 \text { [42] }\end{array}$} & $\begin{array}{l}\text { IG1: BFB ( } \pm \text { retention con- } \\
\text { trol/pain management } \\
\text { techniques) }\end{array}$ & $\begin{array}{l}\text { Baseline, after treatment, } \\
2 \text { months after treatment }\end{array}$ & $\begin{array}{l}\text { Perivaginal sEMG elec- } \\
\text { trodes, visual feedback }\end{array}$ & $\begin{array}{l}\text { Mean } 8 \text { [5-12] sessions (until aim } \\
\text { reached), further data n.a. }\end{array}$ & Clinical (HE: yes) \\
\hline & $\begin{array}{l}\text { IG2: progressive muscle } \\
\text { relaxation ( } \pm \text { retention } \\
\text { control/pain management), } \\
\text { session number same as } \\
\text { yoked partner in BFB group }\end{array}$ & Same as IG1 & - & - & - \\
\hline & $\begin{array}{l}\text { Comparison group: no } \\
\text { intervention (cross-over } \\
\text { after } 2 \text { months) }\end{array}$ & Same as IG1 & - & - & - \\
\hline \multirow[t]{2}{*}{$\begin{array}{l}\text { Hart et al. } \\
1981[43]\end{array}$} & $\begin{array}{l}\text { IG 1: EMG general relax- } \\
\text { ation BFB }\end{array}$ & $\begin{array}{l}\text { Baseline, after treatment } \\
\text { (8 weeks), } 8 \text { weeks after } \\
\text { treatment }\end{array}$ & $\begin{array}{l}\text { SEMG electrodes frontalis } \\
\text { muscle, aural feedback }\end{array}$ & $\begin{array}{l}\text { Mean: } 12.9 \text { [9-15] sessions, } \\
30 \text { min, } 2 \times / \text { week, over } 2 \text { men- } \\
\text { strual cycles }\end{array}$ & Clinical (HE: yes) \\
\hline & $\begin{array}{l}\text { IG2: temperature general } \\
\text { relaxation BFB }\end{array}$ & Same as IG1 & $\begin{array}{l}\text { Skin temperature, vi- } \\
\text { sual + aural feedback }\end{array}$ & Same as IG1 & Same as IG1 \\
\hline \multirow[t]{3}{*}{$\begin{array}{l}\text { Bennink et al. } \\
1982[44]\end{array}$} & $\begin{array}{l}\text { IG1: BFB + general relax- } \\
\text { ation }\end{array}$ & $\begin{array}{l}\text { Baseline (interview), af- } \\
\text { ter first menstrual cycle } \\
\text { (before treatment) and } \\
\sim 1 \text { week after } 3 \text { rd or } 4 \text { th } \\
\text { cycle (post treatment) }\end{array}$ & $\begin{array}{l}\text { sEMG electrodes (lower } \\
\text { abdomen), aural feedback }\end{array}$ & $\begin{array}{l}5 \text { sessions, } 30 \mathrm{~min}, 3 \text { sessions } \\
\text { before, } 2 \text { sessions on first } 2 \text { days } \\
\text { of period }\end{array}$ & Clinical (HE: yes) \\
\hline & $\begin{array}{l}\text { IG2: same general and PF } \\
\text { relaxation training without } \\
\text { BFB }\end{array}$ & Same as IG1 & - & - & - \\
\hline & CG: no intervention & Same as IG1 & - & - & - \\
\hline \multirow[t]{3}{*}{$\begin{array}{l}\text { Vagedes et al. } \\
2019 \text { [45] }\end{array}$} & $\begin{array}{l}\text { IG1: BFB—slow breathing } \\
\text { technique (general relax- } \\
\text { ation) }\end{array}$ & $\begin{array}{l}\text { Baseline-after treatment } \\
\text { (n.a.) }\end{array}$ & $\begin{array}{l}\text { Heart rate variability Qiu } \\
\text { (Biosign) device, visual } \\
\text { feedback }\end{array}$ & $\begin{array}{l}15 \mathrm{~min} / \text { day, } 7 \times / \text { week, over } \\
12 \text { weeks }\end{array}$ & $\begin{array}{l}\text { Home-based, clinical } \\
\text { f/u after } 1,3 \text {, then } \\
\text { every } 4 \text { weeks }\end{array}$ \\
\hline & $\begin{array}{l}\text { IG2: rhythmical massage } \\
\text { (anthroposophic medicine) } \\
30-45 \text { min, } 1 \times / \text { week, } \\
3 \text { months }\end{array}$ & Same as IG1 & - & - & - \\
\hline & $\begin{array}{l}\text { CG: standard care (anal- } \\
\text { gesics, physical exercise, } \\
\text { warmth) }\end{array}$ & Same as IG1 & - & - & - \\
\hline $\begin{array}{l}\text { Starr et al. } \\
2013[46]\end{array}$ & $\begin{array}{l}\text { Complex PF rehabilitation: } \\
\text { instruction, behavioral } \\
\text { management, EGS, BFB }\end{array}$ & $\begin{array}{l}2^{\text {nd }} B F B \text { treatment-after } \\
\text { treatment }\end{array}$ & $\begin{array}{l}\text { sEMG electrodes (abdomi- } \\
\text { nals), vaginal sEMG probe, } \\
\text { anorectal manometry }\end{array}$ & $\begin{array}{l}5-8 \text { sessions ( } 8 \text { if improve- } \\
\text { ment }<80 \% \text { after } 5 \text { sessions), } \\
1 \times / 2 \text { weeks }\end{array}$ & Clinical (HE: yes) \\
\hline \multirow[t]{3}{*}{$\begin{array}{l}\text { Lúcio et al. } \\
2014 \text { [47] }\end{array}$} & $\begin{array}{l}\text { IG1: BFB + PF muscle } \\
\text { training + placebo EGS }\end{array}$ & $\begin{array}{l}\text { Baseline-after treatment } \\
\text { (12 weeks) }\end{array}$ & sEMG vaginal probe & $\begin{array}{l}24 \text { sessions, } 30 \mathrm{~min}, 2 \times / \text { week, } \\
12 \text { weeks }\end{array}$ & Clinical (HE: yes) \\
\hline & $\begin{array}{l}\text { IG2: BFB + PF muscle train- } \\
\text { ing + vaginal EGS ( } 30 \text { min, } \\
2 \times / \text { week, } 12 \text { weeks) }\end{array}$ & Same as IG1 & Same as IG1 & Same as IG1 & Same as IG1 \\
\hline & $\begin{array}{l}\text { IG3: BFB + PF muscle train- } \\
\text { ing + transcutaneous tibial } \\
\text { nerve stimulation ( } 30 \mathrm{~min} \text {, } \\
2 \times / \text { week, } 12 \text { weeks) }\end{array}$ & Same as IG1 & Same as IG1 & Same as IG1 & Same as IG1 \\
\hline \multirow[t]{2}{*}{$\begin{array}{l}\text { Aalaie et al. } \\
2020 \text { [48] }\end{array}$} & $\begin{array}{l}\text { IG1: BFB, } 100 \text { min, } 2 \times \text { I } \\
\text { week, } 6 \text { weeks }+ \text { Kegel } \\
\text { exercises at home }\end{array}$ & $\begin{array}{l}\text { Baseline-2, } 3 \text { months } \\
\text { after treatment }\end{array}$ & sEMG vaginal probe & $\begin{array}{l}12 \text { sessions, } 100 \mathrm{~min}, 2 \times / \text { week, } \\
\text { over } 6 \text { weeks }\end{array}$ & Clinical (HE: yes) \\
\hline & $\begin{array}{l}\text { IG2: vaginal EGS ( } 50 \text { min } \\
\text { of stimulation, } 2 \times / \text { week, } \\
6 \text { weeks) + Kegel exercises } \\
\text { at home }\end{array}$ & Same as IG1 & - & - & - \\
\hline
\end{tabular}




\begin{tabular}{|c|c|c|c|c|c|}
\hline Study & $\begin{array}{l}\text { Interventions performed in } \\
\text { longitudinal study arm }\end{array}$ & Time points of follow-up & BFB device details & $\begin{array}{l}\text { BFB treatment details: number } \\
\text { of sessions, duration per session } \\
\text { (min/, frequency) (times/week), } \\
\text { duration of whole intervention } \\
\text { (weeks, if available) }\end{array}$ & $\begin{array}{l}\text { Setting of BFB inter- } \\
\text { vention (clinical/home- } \\
\text { based), home exer- } \\
\text { cise (=HE) encouraged } \\
\text { (yes/not addressed) }\end{array}$ \\
\hline \multicolumn{6}{|c|}{ Chronic pelvic pain in children } \\
\hline $\begin{array}{l}\text { Hoebeke et al. } \\
2004 \text { [51] }\end{array}$ & $\begin{array}{l}\text { BFB } \pm \text { anticholinergics } \\
(n=13 / 21 \text { with detrusor } \\
\text { hyperactivity) }\end{array}$ & $\begin{array}{l}\text { Baseline, after treatment } \\
\text { (12 weeks) }\end{array}$ & $\begin{array}{l}\text { Anal plug sEMG, visual } \\
\text { BFB }\end{array}$ & $\begin{array}{l}12 \text { sessions, duration n.a., } 1 \times \text { l } \\
\text { week }\end{array}$ & Clinical (HE: n.a.) \\
\hline $\begin{array}{l}\text { Ebiloglu et al. } \\
2016[52]\end{array}$ & BFB & $\begin{array}{l}\text { Baseline, f/u at } 3 \mathrm{rd} \text { and } \\
6 \text { th month (total treatment } \\
\text { time: } 6 \text { months) }\end{array}$ & $\begin{array}{l}\text { Uroflowmeter + sEMG per- } \\
\text { ineal electrodes (external } \\
\text { sphincter), visual feedback }\end{array}$ & $\begin{array}{l}4 \text { sessions, } 10 \mathrm{~min}, 1 \times / \text { week }\left(1^{\text {st }}\right. \\
\text { month), then continued without } \\
\text { BFB, f/u BFB at } 3^{\text {rd }}, 6^{\text {th }} \text { month }\end{array}$ & Clinical (HE: yes) \\
\hline $\begin{array}{l}\text { Ergin et al. } \\
2016 \text { [53] }\end{array}$ & $\mathrm{BFB}$ & $\begin{array}{l}\text { Baseline, after treatment } \\
\text { ( } 6 \text { months) }\end{array}$ & $\begin{array}{l}\text { Uroflowmetry including } \\
\text { sEMG }\end{array}$ & $\begin{array}{l}\geq 6 \text { sessions, over } 6 \text { months, } \\
\text { further data n.a. }\end{array}$ & Clinical (HE: n.a.) \\
\hline \multirow[t]{2}{*}{$\begin{array}{l}\text { Li et al. } 2006 \\
\text { [54] }\end{array}$} & BFB & $\begin{array}{l}\text { Baseline, } f / u \text { after } \\
\sim 12 \text { weeks }\end{array}$ & $\begin{array}{l}\text { Urodynamic system: anal } \\
\text { sEMG probe, abdomi- } \\
\text { nal pressure (intra-anal } \\
\text { balloon catheter) }\end{array}$ & $\begin{array}{l}\text { No. sessions n.a., } 20-30 \mathrm{~min} \text {, } \\
2-3 \times / \text { week, over several weeks }\end{array}$ & Clinical (HE: n.a.) \\
\hline & $\begin{array}{l}\text { CG: healthy controls, no } \\
\text { intervention }\end{array}$ & - & - & - & - \\
\hline \multicolumn{6}{|c|}{ Musculoskeletal, low back pain, myofascial pain } \\
\hline \multirow[t]{2}{*}{$\begin{array}{l}\text { Kent et al. } \\
2015 \text { [55] }\end{array}$} & $\begin{array}{l}\text { IG-BFB: BFB based } \\
\text { movement modifica- } \\
\text { tion + education, guide- } \\
\text { lines-based medical or } \\
\text { physiotherapy care }\end{array}$ & $\begin{array}{l}6 \times \text { during } 10 \text {-weeks } \\
\text { of treatment (baseline, } \\
\text { week } 1,3,6,8,10) \text {, f/u at } \\
\text { week } 12,26,52\end{array}$ & $\begin{array}{l}\text { Motion-sensor movement } \\
\text { biofeedback (ViMove } \\
\text { device), sEMG sensors, } \\
\text { aural + vibrational feed- } \\
\text { back }\end{array}$ & $\begin{array}{l}6 \text { (subacute pain)-8 (chronic } \\
\text { pain) sessions, over } 10 \text { weeks, } \\
\text { frequency n.a. }\end{array}$ & Clinical + home-based \\
\hline & $\begin{array}{l}\text { IG-placebo: } \\
\text { placebo + education, guide- } \\
\text { lines-based medical or } \\
\text { physiotherapy care }\end{array}$ & Same as IG-BFB & - & - & - \\
\hline
\end{tabular}

ment in detail. To provide a better overview, the main conclusions drawn by the respective authors are additionally subsumed in Table 2 .

Only three [16, 17, 19] out of five studies evaluating anorectal pain syndrome provided $p$-values for pain outcomes. Significant anorectal pain relief could be shown, whereby patients who finished had superior results compared to those who discharged themselves before completion of treatment [19]. A large RCT of good quality by Chiarioni et al. 2010 found biofeedback to be superior to electrogalvanic stimulation and local massage therapy both in the short and long term, whereby these differences were only significant in patients with a highly likely levator ani syndrome (tenderness of the levator ani muscle on the rectal examination) [16].

Eleven studies investigated patients with constipation: 2 RCTs of adequate sample size studied patients with dyssynergic defecation [21, 26] and found that biofeedback significantly decreased abdominal pain compared to laxatives (polyethylene glycol) [21, 26] with long-term effects and huge effect sizes significantly different from zero [21]. The same two RCTs found biofeedback superior to laxatives in terms of constipation symptom improvement with very large effect sizes [26].
Several of the remaining nine non-RCTs found pain [22-24, 27, 29] and constipation symptoms [22, 23, $25,27,29,31]$ improved after biofeedback, at least for certain subgroups. Studies showed contradictory results regarding the question of whether biofeedback only benefited patients with PF dyssynergia or also patients with prolonged transit time. Some studies found that biofeedback improved (long term) symptoms for pelvic floor dyssynergia [22, 24, 28] but not for slow transit constipation [24, 28], others found that both phenotypes benefited equally from treatment $[23,25,27]$.

With respect to the 11 studies on female chronic pelvic pain, several could improve pain [37-40,48] or symptoms [38, 39, 41, 43, 44, 46-48], at least in the longer term. Again, several studies lacked $p$-values or measures of clinical relevance.

The 4 urogenital studies on children and adolescents and 4 studies on men with chronic prostatitis mostly found improvements in pain [32, 34-36, 51, 52, 54] and urological symptoms [32, 34-36, 52-54], with medium to huge effect sizes in Yang et al. [35].

\section{Effect of biofeedback interventions on quality of life}

Nine studies used biofeedback to improve pelvic floor function and found a significant improvement in the 
Table 5 Primary outcome: effect on pain and overall symptoms

\begin{tabular}{|c|c|c|c|c|c|c|c|}
\hline Study & $\begin{array}{l}n \text { (total) } \\
\text { (group 1, } \\
\text { 2) }\end{array}$ & Group 1 (n1) & Group 2 (n2) & Outcome measure & $\begin{array}{l}\text { Mean difference: } \\
\text { Group } 2 \text { minus } \\
\text { Group } 1 \text { [Cl] }\end{array}$ & $\begin{array}{l}\text { Effect size } \\
\text { [strength] }\end{array}$ & $\begin{array}{l}P \text {-value (for } \\
\text { difference in } \\
\text { means) }\end{array}$ \\
\hline \multicolumn{8}{|c|}{ Anorectal pain syndrome } \\
\hline \multirow{22}{*}{$\begin{array}{l}\text { Chiarioni } \\
\text { et al. } 2010 \\
\text { [16] }\end{array}$} & 104 & $\begin{array}{l}\text { IG1 (BFB) in pat. w LAS } \\
\text { (52) }\end{array}$ & IG2 (EGS) in pat. w LAS (52) & $\begin{array}{l}\text { SR: \% pat. w adequate pain } \\
\text { relief after } 1 \text { month }\end{array}$ & -26.9 & n.a. & $p<0.01^{f}$ \\
\hline & 104 & $\begin{array}{l}\text { IG1 (BFB) in pat. w LAS } \\
\text { (52) }\end{array}$ & IG2 (EGS) in pat. w LAS (52) & $\begin{array}{l}\text { SR: \% pat. w adequate pain } \\
\text { relief after } 3 \text { months }\end{array}$ & -18.9 & n.a. & $p<0.01^{f}$ \\
\hline & 104 & $\begin{array}{l}\text { IG1 (BFB) in pat. w LAS } \\
\text { (52) }\end{array}$ & IG2 (EGS) in pat. w LAS (52) & $\begin{array}{l}\text { SR: \% pat. w adequate pain } \\
\text { relief after } 6 \text { months }\end{array}$ & -31.2 & n.a. & $p<0.01^{f}$ \\
\hline & 104 & $\begin{array}{l}\text { IG1 (BFB) in pat. w LAS } \\
\text { (52) }\end{array}$ & IG2 (EGS) in pat. w LAS (52) & $\begin{array}{l}\text { SR: \% pat. w adequate pain } \\
\text { relief after } 12 \text { months }\end{array}$ & -31.2 & n.a. & $p<0.01^{f}$ \\
\hline & 105 & $\begin{array}{l}\text { IG1 (BFB) in pat. w LAS } \\
\text { (52) }\end{array}$ & $\begin{array}{l}\text { IG3 (massage) in patients } \\
\text { with LAS (53) }\end{array}$ & $\begin{array}{l}\text { SR: \% pat. w adequate pain } \\
\text { relief after } 1 \text { month }\end{array}$ & -31.3 & n.a. & $p<0.01^{f}$ \\
\hline & 105 & $\begin{array}{l}\text { IG1 (BFB) in pat. w LAS } \\
\text { (52) }\end{array}$ & $\begin{array}{l}\text { IG3 (massage) in patients } \\
\text { with LAS (53) }\end{array}$ & $\begin{array}{l}\text { SR: } \% \text { pat. w adequate pain } \downarrow \\
\text { after } 3,6,12 \text { months }\end{array}$ & -36.9 & n.a. & $p<0.01^{f}$ \\
\hline & 104 & $\begin{array}{l}\text { BFB in pat. w "high likely" } \\
\text { LAS (n.a.) }\end{array}$ & $\begin{array}{l}\text { EGS in pat. w "high likely" } \\
\text { LAS (n.a.) }\end{array}$ & \multirow[t]{2}{*}{$\begin{array}{l}\text { SR: \% pat. w adequate pain } \\
\text { relief after } 1,3,6,12 \text { months }\end{array}$} & \multirow[t]{2}{*}{$\begin{array}{l}\text { In favour of BFB } \\
\text { group }\end{array}$} & n.a. & $p<0.025^{f}$ \\
\hline & 105 & $\begin{array}{l}\text { BFB in pat. w "high likely" } \\
\text { LAS (n.a.) }\end{array}$ & $\begin{array}{l}\text { Massage in pat. w "high } \\
\text { likely" LAS (n.a.) }\end{array}$ & & & n.a. & $p<0.025^{f}$ \\
\hline & 104 & $\begin{array}{l}\text { BFB in pat. w "possible" } \\
\text { LAS (n.a.) }\end{array}$ & $\begin{array}{l}\text { EGS in pat. w "possible" LAS } \\
\text { (n.a.) }\end{array}$ & \multirow[t]{2}{*}{$\begin{array}{l}\text { SR: } \% \text { pat. } w \text { adequate pain } \\
\text { relief after } 1,3,6,12 \text { months }\end{array}$} & - & n.a. & $p>0.025^{f}$ \\
\hline & 105 & $\begin{array}{l}\text { BFB in pat. w "possible" } \\
\text { LAS (n.a.) }\end{array}$ & $\begin{array}{l}\text { Massage in pat. w "possible" } \\
\text { LAS (n.a.) }\end{array}$ & & - & n.a. & $p>0.025^{f}$ \\
\hline & 104 & $\begin{array}{l}\text { BFB in pat. w "high likely" } \\
\text { LAS (n.a.) }\end{array}$ & $\begin{array}{l}\text { EGS in pat. w "high likely" } \\
\text { LAS (n.a.) }\end{array}$ & \multirow{4}{*}{$\begin{array}{l}\text { Subjective change in pain to } \\
\text { baseline, ordinal scale }[-2 \text { to } \\
+3:-2 \text { "a lot worse" to }+3 \text { "a } \\
\text { lot better/cured"] after } 1,3, \\
6 \text { months }\end{array}$} & \multirow[t]{2}{*}{$\begin{array}{l}\text { In favour of BFB } \\
\text { group }\end{array}$} & n.a. & $p<0.025^{d}$ \\
\hline & 105 & $\begin{array}{l}\text { BFB in pat. w "high likely" } \\
\text { LAS (n.a.) }\end{array}$ & $\begin{array}{l}\text { Massage in pat. w "high } \\
\text { likely" LAS (n.a.) }\end{array}$ & & & n.a. & $p<0.025^{d}$ \\
\hline & 104 & $\begin{array}{l}\text { BFB in pat. w "possible" } \\
\text { LAS (n.a.) }\end{array}$ & $\begin{array}{l}\text { EGS in pat. w "possible" LAS } \\
\text { (n.a.) }\end{array}$ & & - & n.a. & $p>0.025^{d}$ \\
\hline & 105 & $\begin{array}{l}\text { BFB in pat. w "possible" } \\
\text { LAS (n.a.) }\end{array}$ & $\begin{array}{l}\text { Massage in pat. w "possible" } \\
\text { LAS (n.a.) }\end{array}$ & & - & n.a. & $p>0.025^{d}$ \\
\hline & 104 & $\begin{array}{l}\text { BFB in pat. w "high likely" } \\
\text { LAS (n.a.) }\end{array}$ & $\begin{array}{l}\text { EGS in pat. w "high likely" } \\
\text { LAS (n.a.) }\end{array}$ & \multirow{4}{*}{$\begin{array}{l}\text { Number of days/months with } \\
\text { rectal pain as stated in symp- } \\
\text { tom log ( } 0-30 \text { days) after } 1,3 \text {, } \\
6 \text { months }\end{array}$} & \multirow[t]{2}{*}{$\begin{array}{l}\text { In favour of BFB } \\
\text { group }\end{array}$} & n.a. & $p<0.025^{d}$ \\
\hline & 105 & $\begin{array}{l}\text { BFB in pat. w "high likely" } \\
\text { LAS (n.a.) }\end{array}$ & $\begin{array}{l}\text { Massage in pat. w "high } \\
\text { likely" LAS (n.a.) }\end{array}$ & & & n.a. & $p<0.025^{d}$ \\
\hline & 104 & $\begin{array}{l}\text { BFB in pat. w "possible" } \\
\text { LAS (n.a.) }\end{array}$ & $\begin{array}{l}\text { EGS in pat. w "possible" LAS } \\
\text { (n.a.) }\end{array}$ & & - & n.a. & $p>0.025^{d}$ \\
\hline & 105 & $\begin{array}{l}\text { BFB in pat. w "possible" } \\
\text { LAS (n.a.) }\end{array}$ & $\begin{array}{l}\text { Massage in pat. w "possible" } \\
\text { LAS (n.a.) }\end{array}$ & & - & n.a. & $p>0.025^{d}$ \\
\hline & 104 & $\begin{array}{l}\text { BFB in pat. w "high likely" } \\
\text { LAS (n.a.) }\end{array}$ & $\begin{array}{l}\text { EGS in pat. w "high likely" } \\
\text { LAS (n.a.) }\end{array}$ & \multirow{4}{*}{$\begin{array}{l}\text { Pain: VAS }(0-10 \mathrm{~cm}) \text {, average } \\
\text { value of worst pain/wk, after } \\
1,3,6 \text { months }\end{array}$} & \multirow[t]{2}{*}{$\begin{array}{l}\text { In favour of BFB } \\
\text { group }\end{array}$} & n.a. & $p<0.025^{d}$ \\
\hline & 105 & $\begin{array}{l}\text { BFB in pat. w "high likely" } \\
\text { LAS (n.a.) }\end{array}$ & $\begin{array}{l}\text { Massage in pat. w "high } \\
\text { likely" LAS (n.a.) }\end{array}$ & & & n.a. & $p<0.025^{d}$ \\
\hline & 104 & $\begin{array}{l}\text { BFB in pat. w "possible" } \\
\text { LAS (n.a.) }\end{array}$ & $\begin{array}{l}\text { EGS in pat. w "possible" LAS } \\
\text { (n.a.) }\end{array}$ & & - & n.a. & $p>0.025^{d}$ \\
\hline & 105 & $\begin{array}{l}\text { BFB in pat. w "possible" } \\
\text { LAS (n.a.) }\end{array}$ & $\begin{array}{l}\text { Massage in pat. w "possible" } \\
\text { LAS (n.a.) }\end{array}$ & & - & n.a. & $p>0.025^{d}$ \\
\hline \multirow{2}{*}{$\begin{array}{l}\text { Heah et al. } \\
1997[17]\end{array}$} & 16 & Study group pre-BFB (16) & Study group post-BFB (16) & Pain VAS (0-10) & -6 & n.a. & $p<0.02$ \\
\hline & 16 & Study group pre-BFB (16) & Study group post-BFB (16) & $\begin{array}{l}\text { SR: \% pat. needing anal- } \\
\text { gesics }\end{array}$ & -87.5 & n.a. & $p<0.03$ \\
\hline \multirow[t]{3}{*}{$\begin{array}{l}\text { Ger et al. } \\
1993[18]\end{array}$} & 14 & $n / a$ & $\begin{array}{l}\text { IG2 (BFB group) post-BFB } \\
\text { (14) }\end{array}$ & $\begin{array}{l}\text { SR: \% pat. w complete pain } \\
\text { relief }\end{array}$ & 14.3 & n.a. & n.a. \\
\hline & 14 & $n / a$ & $\begin{array}{l}\text { IG2 (BFB group) post-BFB } \\
\text { (14) }\end{array}$ & $\begin{array}{l}\text { SR: \% pat. w improved pain } \\
\text { frequency/intensity }\end{array}$ & 28.6 & n.a. & n.a. \\
\hline & 14 & $n / a$ & $\begin{array}{l}\text { IG2 (BFB group) post-BFB } \\
\text { (14) }\end{array}$ & SR: \% pat. w no improvement & 57.1 & n.a. & n.a. \\
\hline
\end{tabular}




\section{review article}

Table 5 (Continued)

\begin{tabular}{|c|c|c|c|c|c|c|c|}
\hline Study & $\begin{array}{l}n \text { (total) } \\
\text { (group 1, } \\
\text { 2) }\end{array}$ & Group 1 (n1) & Group 2 (n2) & Outcome measure & $\begin{array}{l}\text { Mean difference: } \\
\text { Group } 2 \text { minus } \\
\text { Group } 1[\mathrm{Cl}]\end{array}$ & $\begin{array}{l}\text { Effect size } \\
\text { [strength] }\end{array}$ & $\begin{array}{l}P \text {-value (for } \\
\text { difference in } \\
\text { means) }\end{array}$ \\
\hline \multirow[t]{3}{*}{$\begin{array}{l}\text { Gilliland et al. } \\
\text { 1997a [19] }\end{array}$} & 75 & $\begin{array}{l}\text { BFB in pat. w rectal pain } \\
\text { only (47) }\end{array}$ & $\begin{array}{l}\text { BFB in pat. with rectal pain } \\
\text { and constipation (28) }\end{array}$ & \multirow[t]{3}{*}{$\begin{array}{l}\text { SR: \% patients reporting } \\
\text { symptom improvement }\end{array}$} & -4.02 & n.a. & $p=0.81$ \\
\hline & 46 & $\begin{array}{l}\text { BFB subgroup of Group 1: } \\
\text { pat. who finished trial (7) }\end{array}$ & $\begin{array}{l}\text { BFB subgroup of Group 1: } \\
\text { self-discharged early ( } 39 \text { ) }\end{array}$ & & -57.5 & n.a. & $p<0.01$ \\
\hline & 28 & $\begin{array}{l}\text { BFB subgroup of Group } \\
\text { 2: pat. who finished trial } \\
\text { (n.a.) }\end{array}$ & $\begin{array}{l}\text { BFB subgroup of Group 2: } \\
\text { self-discharged early (n.a.) }\end{array}$ & & -46.7 & n.a. & $p<0.05$ \\
\hline \multicolumn{8}{|c|}{ Constipation, dyssynergic defecation } \\
\hline \multirow[t]{7}{*}{$\begin{array}{l}\text { Chiarioni et } \\
\text { al. } 2006 \text { [21] }\end{array}$} & 54 & $\begin{array}{l}\text { IG1 (BFB) pre-treatment } \\
\text { (54) }\end{array}$ & $\begin{array}{l}\text { IG1 (BFB) } 6 \text { months after } \\
\text { starting treatment (54) }\end{array}$ & \multirow[t]{4}{*}{$\begin{array}{l}\text { Frequency of abdominal } \\
\text { pain/wk (symptom diary) }\end{array}$} & $\begin{array}{l}-0.69 \\
{[-0.74 ;-0.64]}\end{array}$ & $-5.86[H]$ & $p<0.01^{c}$ \\
\hline & 54 & $\begin{array}{l}\text { IG1 (BFB) pre-treatment } \\
\text { (54) }\end{array}$ & $\begin{array}{l}\text { IG1 (BFB) } 12 \text { months after } \\
\text { starting treatment (54) }\end{array}$ & & $\begin{array}{l}-0.68 \\
{[-0.73 ;-0.63]}\end{array}$ & $-5.78[\mathrm{H}]$ & $p<0.01^{c}$ \\
\hline & 109 & $\begin{array}{l}\text { IG1 (BFB) } 6 \text { months after } \\
\text { starting treatment (54) }\end{array}$ & $\begin{array}{l}\text { IG2 (laxatives) } 6 \text { months after } \\
\text { starting treatment (55) }\end{array}$ & & $\begin{array}{l}0.63 \\
{[0.57 ; 0.69]}\end{array}$ & $4.85[\mathrm{H}]$ & $p<0.01^{c}$ \\
\hline & 109 & $\begin{array}{l}\text { IG1 (BFB) } 6 \text { months after } \\
\text { starting treatment (54) }\end{array}$ & $\begin{array}{l}\text { IG2 (laxatives) } 12 \text { months } \\
\text { after starting treatment (55) }\end{array}$ & & $\begin{array}{l}0.58 \\
{[0.52 ; 0.64]}\end{array}$ & $4.26[\mathrm{H}]$ & $p<0.01^{c}$ \\
\hline & 109 & $\begin{array}{l}\text { IG1 (BFB) } 6+12 \text { months } \\
\text { after starting treatment } \\
\text { (54) }\end{array}$ & $\begin{array}{l}\text { IG2 (laxatives) } 6+12 \text { months } \\
\text { after starting treatment (55) }\end{array}$ & \multirow[t]{3}{*}{$\begin{array}{l}\text { SR: \% patients reporting } \\
\text { symptom improvement (4 out } \\
\text { of a scale } 0-4 \text { ) }\end{array}$} & -57.8 & n.a. & n.a. \\
\hline & 54 & $n / a$ & $\begin{array}{l}\text { IG1 (BFB) } 6+12 \text { months after } \\
\text { starting treatment (54) }\end{array}$ & & 79.6 & n.a. & n.a. \\
\hline & 54 & $n / a$ & $\begin{array}{l}\text { IG1 (BFB) } 24 \text { months after } \\
\text { starting treatment (54) }\end{array}$ & & 81.5 & n.a. & n.a. \\
\hline \multirow{9}{*}{$\begin{array}{l}\text { Koutsomanis } \\
\text { et al. } 1994 \\
\text { [22] }\end{array}$} & 20 & Study group pre-BFB (20) & $\begin{array}{l}\text { Study group immediately } \\
\text { post-BFB (20) }\end{array}$ & \multirow[t]{3}{*}{$\begin{array}{l}\text { SR: \% patients reporting ab- } \\
\text { dominal pain } \geq 1 / \text { week }\end{array}$} & -20 & n.a. & $\geq 0.05^{b}$ \\
\hline & 18 & Study group pre-BFB (20) & $\begin{array}{l}\text { Study group } 6 \text { weeks after } \\
\text { starting BFB (18) }\end{array}$ & & -13.3 & n.a. & $\geq 0.05^{b}$ \\
\hline & 20 & Study group pre-BFB (20) & $\begin{array}{l}\text { Study group 6-12 months } \\
\text { after 6-wk-f/u (20) }\end{array}$ & & -10 & n.a. & $\geq 0.05^{b}$ \\
\hline & 20 & Study group pre-BFB (20) & $\begin{array}{l}\text { Study group immediately } \\
\text { post-BFB (20) }\end{array}$ & \multirow{3}{*}{$\begin{array}{l}\text { Weekly total pain score } \\
\text { (daily pain score: } 0=\text { none, } \\
3=\text { severe) }\end{array}$} & -5.5 & n.a. & $\geq 0.05^{b}$ \\
\hline & 18 & Study group pre-BFB (20) & $\begin{array}{l}\text { Study group } 6 \text { weeks after } \\
\text { starting BFB (18) }\end{array}$ & & -8 & n.a. & $p<0.01^{b}$ \\
\hline & 20 & Study group pre-BFB (20) & $\begin{array}{l}\text { Study group 6-12 months } \\
\text { after 6-wk-f/u (20) }\end{array}$ & & -9 & n.a. & $p<0.01^{b}$ \\
\hline & 20 & Study group pre-BFB (20) & $\begin{array}{l}\text { Study group immediately } \\
\text { post-BFB (20) }\end{array}$ & \multirow{3}{*}{$\begin{array}{l}\text { Weekly overall symptom } \\
\text { score (daily score: } 0=\text { better, } \\
1=\text { same, } 2=\text { worse) }\end{array}$} & -4 & n.a. & $p<0.01^{b}$ \\
\hline & 18 & Study group pre-BFB (20) & $\begin{array}{l}\text { Study group } 6 \text { weeks after } \\
\text { starting BFB (18) }\end{array}$ & & -4 & n.a. & $p<0.01^{b}$ \\
\hline & 20 & Study group pre-BFB (20) & $\begin{array}{l}\text { Study group 6-12 months } \\
\text { after 6-wk-f/u (20) }\end{array}$ & & -6 & n.a. & $p<0.01^{b}$ \\
\hline \multirow{6}{*}{$\begin{array}{l}\text { Chiotakakou- } \\
\text { Faliakou et al. } \\
1998 \text { [23] }\end{array}$} & 100 & $\begin{array}{l}\text { Study group pre-BFB } \\
(100)\end{array}$ & Study group post-BFB (100) & \multirow[t]{2}{*}{$\begin{array}{l}\text { SR: \% patients with abdominal } \\
\text { pain }\end{array}$} & -16 & n.a. & $p=0.003^{f}$ \\
\hline & 100 & $\begin{array}{l}\text { Study group pre-BFB } \\
(100)\end{array}$ & $\begin{array}{l}\text { Study group long-term (mean } \\
23.4 \text { months) post-BFB (100) }\end{array}$ & & -20 & n.a. & $\begin{array}{l}p= \\
0.0004^{f}\end{array}$ \\
\hline & 100 & $n / a$ & Study group post-BFB (100) & \multirow{2}{*}{$\begin{array}{l}\text { SR: \% patients stating BFB } \\
\text { improved bowel symptoms (a } \\
\text { little-a lot) }\end{array}$} & 66 & n.a. & n.a. \\
\hline & 100 & $n / a$ & $\begin{array}{l}\text { Study group long-term (mean } \\
23.4 \text { months) post-BFB (100) }\end{array}$ & & 55 & n.a. & n.a. \\
\hline & 100 & $n / a$ & $\begin{array}{l}\text { Study group post-BFB (100) } \\
\text { in pat. w constipation }\end{array}$ & \multirow{2}{*}{$\begin{array}{l}\text { SR: \% patients reporting } \\
\text { sonstipation symptom im- } \\
\text { provement (a little-a lot) }\end{array}$} & 50 & n.a. & n.a. \\
\hline & 100 & $n / a$ & $\begin{array}{l}\text { Study group long-term } \\
\text { (23.4 months) post-BFB } \\
\text { (100) in pat. w constipation }\end{array}$ & & 57 & n.a. & n.a. \\
\hline
\end{tabular}


Table 5 (Continued)

\begin{tabular}{|c|c|c|c|c|c|c|c|}
\hline Study & $\begin{array}{l}n \text { (total) } \\
\text { (group 1, } \\
\text { 2) }\end{array}$ & Group 1 (n1) & Group 2 (n2) & Outcome measure & $\begin{array}{l}\text { Mean difference: } \\
\text { Group } 2 \text { minus } \\
\text { Group } 1[\mathrm{Cl}]\end{array}$ & $\begin{array}{l}\text { Effect size } \\
\text { [strength] }\end{array}$ & $\begin{array}{l}P \text {-value (for } \\
\text { difference in } \\
\text { means) }\end{array}$ \\
\hline \multirow[t]{3}{*}{$\begin{array}{l}\text { Battaglia et } \\
\text { al. } 2004 \text { [24] }\end{array}$} & 14 & $\begin{array}{l}\text { Subgroup with PF dyssyn- } \\
\text { ergia pre-BFB (14) }\end{array}$ & $\begin{array}{l}\text { This subgroup } 3+12 \text { months } \\
\text { after BFB (14) }\end{array}$ & \multirow[t]{3}{*}{$\begin{array}{l}\text { SR: \% patients with abdomi- } \\
\text { nal pain }\end{array}$} & -21.4 & n.a. & n.a. \\
\hline & 10 & $\begin{array}{l}\text { Subgroup with slow tran- } \\
\text { sit constipation pre-BFB } \\
\text { (10) }\end{array}$ & $\begin{array}{l}\text { This subgroup } 3 \text { months } \\
\text { post-BFB (10) }\end{array}$ & & -80 & n.a. & n.a. \\
\hline & 10 & $\begin{array}{l}\text { Subgroup with slow tran- } \\
\text { sit constipation pre-BFB } \\
\text { (10) }\end{array}$ & $\begin{array}{l}\text { This subgroup } 12 \text { months } \\
\text { post-BFB (10) }\end{array}$ & & -20 & n.a. & n.a. \\
\hline \multirow{6}{*}{$\begin{array}{l}\text { Wang et al. } \\
2003 \text { [25] }\end{array}$} & 50 & Study group pre-BFB (50) & Study group post-BFB (50) & \multirow{2}{*}{$\begin{array}{l}\text { SR: \% patients with perianal } \\
\text { pain at defacation }\end{array}$} & -28 & n.a. & $\geq 0.05^{\mathrm{a}}$ \\
\hline & 50 & Study group pre-BFB (50) & $\begin{array}{l}\text { Study group 1-year post-BFB } \\
\text { (50) }\end{array}$ & & -38 & n.a. & $\geq 0.05^{\mathrm{a}}$ \\
\hline & 50 & $n / a$ & Study group post-BFB (50) & \multirow{4}{*}{$\begin{array}{l}\text { SR: \% patients reporting } \\
\text { overall symptom improvement }\end{array}$} & 62 & n.a. & n.a. \\
\hline & 8 & $n / a$ & $\begin{array}{l}\text { Pat. w slow transit constipa- } \\
\text { tion post-BFB (8) }\end{array}$ & & 37.5 & n.a. & n.a. \\
\hline & 36 & $n / a$ & $\begin{array}{l}\text { Pat. w PF dysfunction post- } \\
\text { BFB (36) }\end{array}$ & & 72.2 & n.a. & n.a. \\
\hline & 6 & $n / a$ & $\begin{array}{l}\text { Pat. w combined PF dysf. } \\
\text { +slow transit post-BFB (6) }\end{array}$ & & 33.3 & n.a. & n.a. \\
\hline \multirow{6}{*}{$\begin{array}{l}\text { Ba-Bai-Ke- } \\
\text { Re et al. } \\
2014 \text { [26] }\end{array}$} & 88 & $\begin{array}{l}\text { IG1 (BFB) } 1 \text { month post- } \\
\text { treatment (44) }\end{array}$ & $\begin{array}{l}\text { IG2 (laxatives) } 1 \text { month post- } \\
\text { treatment (44) }\end{array}$ & \multirow[t]{3}{*}{$\begin{array}{l}\text { SR; \% of patients with peri- } \\
\text { anal pain at defecation }\end{array}$} & 36.4 & n.a. & $0.0006^{a}$ \\
\hline & 88 & $\begin{array}{l}\text { IG1 (BFB) } 3 \text { months post- } \\
\text { treatment (44) }\end{array}$ & $\begin{array}{l}\text { IG2 (laxatives) } 3 \text { months post- } \\
\text { treatment (44) }\end{array}$ & & 20.5 & n.a. & $0.0534^{\mathrm{a}}$ \\
\hline & 88 & $\begin{array}{l}\text { IG1 (BFB) } 6 \text { months post- } \\
\text { treatment (44) }\end{array}$ & $\begin{array}{l}\text { IG2 (laxatives) } 6 \text { months post- } \\
\text { treatment (44) }\end{array}$ & & 20.5 & n.a. & $0.0375^{a}$ \\
\hline & 88 & $\begin{array}{l}\text { IG1 (BFB) } 1 \text { month post- } \\
\text { treatment (44) }\end{array}$ & $\begin{array}{l}\text { IG2 (laxatives) } 1 \text { month post- } \\
\text { treatment (44) }\end{array}$ & \multirow{3}{*}{$\begin{array}{l}\text { Symptom score: Wexner } \\
\text { constipation summary score } \\
(0-30=\text { worst) [62] }\end{array}$} & $\begin{array}{l}-6.00 \\
{[-7.41 ;-4.59]}\end{array}$ & $-1.45[\mathrm{VL}]$ & $p<0.001^{a}$ \\
\hline & 88 & $\begin{array}{l}\text { IG1 (BFB) } 3 \text { months post- } \\
\text { treatment (44) }\end{array}$ & $\begin{array}{l}\text { IG2 (laxatives) } 3 \text { months post- } \\
\text { treatment (44) }\end{array}$ & & $\begin{array}{l}-5.00 \\
{[-6.21 ;-3.78]}\end{array}$ & $-1.40[\mathrm{VL}]$ & $p<0.001^{a}$ \\
\hline & 88 & $\begin{array}{l}\text { IG1 (BFB) } 6 \text { months post- } \\
\text { treatment (44) }\end{array}$ & $\begin{array}{l}\text { IG2 (laxatives) } 6 \text { months post- } \\
\text { treatment (44) }\end{array}$ & & $\begin{array}{l}-6.00 \\
{[-7.11 ;-4.89]}\end{array}$ & $-1.84[\mathrm{VL}]$ & $p<0.001^{a}$ \\
\hline \multirow[t]{9}{*}{$\begin{array}{l}\text { Roy et al. } \\
2000 \text { [27] }\end{array}$} & 26 & $\begin{array}{l}\text { Pre-BFB in pat. w con- } \\
\text { stipation, attributed to } \\
\text { hysterectomy by patient } \\
\text { (26) }\end{array}$ & $\begin{array}{l}\text { Post-BFB in patients with } \\
\text { constipation, attributed to } \\
\text { hysterectomy by patient ( } 26 \text { ) }\end{array}$ & \multirow[t]{8}{*}{$\begin{array}{l}\text { SR: \% of patients with abdo- } \\
\text { minal pain }\end{array}$} & -23.1 & n.a. & n.a. \\
\hline & 26 & $\begin{array}{l}\text { Pre-BFB in pat. w con- } \\
\text { stipation, attributed to } \\
\text { hysterectomy (26) }\end{array}$ & $\begin{array}{l}\text { Long-term (28 months) post- } \\
\text { BFB in patients with consti- } \\
\text { pation, attributed to hysterec- } \\
\text { tomy (26) }\end{array}$ & & -11.5 & n.a. & n.a. \\
\hline & 27 & $\begin{array}{l}\text { Pre-BFB in pat. w consti- } \\
\text { pation, not attributed to } \\
\text { hysterectomy (27) }\end{array}$ & $\begin{array}{l}\text { Post-BFB in pat. w con- } \\
\text { stipation, not attributed to } \\
\text { hysterectomy (27) }\end{array}$ & & -29.6 & n.a. & n.a. \\
\hline & 27 & $\begin{array}{l}\text { Pre-BFB in pat. } w \text { consti- } \\
\text { pation, not attributed to } \\
\text { hysterectomy (27) }\end{array}$ & $\begin{array}{l}\text { Long-term ( } 28 \text { months) post- } \\
\text { BFB in pat. w constipation, } \\
\text { not attributed to hysterec- } \\
\text { tomy ( } 27)\end{array}$ & & -29.6 & n.a. & n.a. \\
\hline & 25 & $\begin{array}{l}\text { Pre-BFB in pat. w con- } \\
\text { stipation, no history of } \\
\text { hysterectomy (25) }\end{array}$ & $\begin{array}{l}\text { Post-BFB in pat. } \\
\text { w constipation, } \\
\text { no history of hysterectomy } \\
(25)\end{array}$ & & -28.0 & n.a. & n.a. \\
\hline & 25 & $\begin{array}{l}\text { Pre-BFB in pat. w con- } \\
\text { stipation, no history of } \\
\text { hysterectomy (25) }\end{array}$ & $\begin{array}{l}\text { Long-term (28 months) post- } \\
\text { BFB in pat. w constipation, no } \\
\text { history of hysterectomy (25) }\end{array}$ & & -36.0 & n.a. & n.a. \\
\hline & 78 & $\begin{array}{l}\text { Pre-BFB in all pat. w } \\
\text { constipation (78) }\end{array}$ & $\begin{array}{l}\text { Post-BFB in all pat. w consti- } \\
\text { pation (78) }\end{array}$ & & -27.0 & n.a. & n.a. \\
\hline & 78 & $\begin{array}{l}\text { Pre-BFB in all pat. w } \\
\text { constipation (78) }\end{array}$ & $\begin{array}{l}\text { Long-term ( } 28 \text { months) post- } \\
\text { BFB in all pat. w constipation } \\
\text { (78) }\end{array}$ & & -25.6 & n.a. & n.a. \\
\hline & 78 & $n / a$ & $\begin{array}{l}28 \text { months post-BFB in all } \\
\text { pat. w constipation (78) }\end{array}$ & $\begin{array}{l}\text { SR: \% patients reporting } \\
\text { constipation symptom im- } \\
\text { provement }\end{array}$ & 61.5 & n.a. & n.a. \\
\hline
\end{tabular}




\section{review article}

Table 5 (Continued)

\begin{tabular}{|c|c|c|c|c|c|c|c|}
\hline Study & $\begin{array}{l}n \text { (total) } \\
\text { (group 1, } \\
\text { 2) }\end{array}$ & Group 1 (n1) & Group 2 (n2) & Outcome measure & $\begin{array}{l}\text { Mean difference: } \\
\text { Group } 2 \text { minus } \\
\text { Group } 1[\mathrm{Cl}]\end{array}$ & $\begin{array}{l}\text { Effect size } \\
\text { [strength] }\end{array}$ & $\begin{array}{l}P \text {-value (for } \\
\text { difference in } \\
\text { means) }\end{array}$ \\
\hline $\begin{array}{l}\text { Chiarioni et } \\
\text { al. } 2005 \text { [28] }\end{array}$ & 41 & $\begin{array}{l}\text { Subgroup with PF dys- } \\
\text { function after } 1,6,12 \text {, } \\
24 \text { months after BFB ( } 52 \text {, } \\
50,49,45)\end{array}$ & $\begin{array}{l}\text { Subgroup with slow tran- } \\
\text { sit only, after } 1,6,12 \text {, } \\
24 \text { months after BFB ( } 52 \text {, } \\
50,49,45)\end{array}$ & $\begin{array}{l}\text { Pain frequency (in favour of } \\
\text { subgroup PF dyssynergia) }\end{array}$ & n.a. & n.a. & $p<0.05^{a}$ \\
\hline \multirow[t]{2}{*}{$\begin{array}{l}\text { Zhu et al. } \\
2011 \text { [29] }\end{array}$} & 36 & Study group pre-BFB (36) & Study group post-BFB (36) & $\begin{array}{l}\text { SF-36 subscale pain (0-100: } \\
\text { best) }\end{array}$ & $\begin{array}{l}10.3 \\
{[-1.31 ; 21.91]}\end{array}$ & $0.48[S]$ & $p=0.001^{a}$ \\
\hline & 36 & Study group pre-BFB (36) & Study group post-BFB (36) & $\begin{array}{l}\text { Symptom score } \\
(0-15: 0=\text { none, } 3=\text { severe } \\
\text { for } 5 \text { symptoms })\end{array}$ & $\begin{array}{l}-5.77 \\
{[-7.29 ;-4.25]}\end{array}$ & $-2.04[H]$ & $p<0.001^{a}$ \\
\hline \multirow[t]{9}{*}{$\begin{array}{l}\text { Gilliland et al. } \\
\text { 1997b [30] }\end{array}$} & 178 & $n / a$ & Study group post-BFB (178) & $\begin{array}{l}\text { SR: \% patients with } \geq 3 \text { bowel } \\
\text { movements/wk without aid } \\
\text { ("complete success") }\end{array}$ & 35.0 & n.a. & n.a. \\
\hline & 178 & $n / a$ & Study group post-BFB (178) & $\begin{array}{l}\text { SR: \% patients with }<3 \text { bowel } \\
\text { movements/wk with reduced } \\
\text { aid ("partial success") }\end{array}$ & 13.5 & n.a. & n.a. \\
\hline & 178 & $n / a$ & Study group post-BFB (178) & $\begin{array}{l}\text { SR: \% patients with no im- } \\
\text { provement ("failed") }\end{array}$ & 51.1 & n.a. & n.a. \\
\hline & 60 & $n / a$ & $\begin{array}{l}\text { Study group post-BFB, pat. } \\
\text { attended } 2-4 \text { sessions ( } 60 \text { ) }\end{array}$ & \multirow{6}{*}{$\begin{array}{l}\text { SR: \% patients with } \geq 3 \text { bowel } \\
\text { movements/wk without aid } \\
\text { ("complete success") }\end{array}$} & 18.0 & n.a. & n.a. \\
\hline & 118 & $n / a$ & $\begin{array}{l}\text { Study group post-BFB, pat. } \\
\text { attended } \geq 5 \text { sessions (118) }\end{array}$ & & 44.0 & n.a. & n.a. \\
\hline & 178 & $n / a$ & $\begin{array}{l}\text { Study group post-BFB, pat. } \\
\text { attended } 2-4 \text { sessions ( } 60 \text { ) }\end{array}$ & & -26.0 & n.a. & $p<0.001$ \\
\hline & 52 & $n / a$ & $\begin{array}{l}\text { Study group post-BFB, pat. } \\
\text { completed BFB (52) }\end{array}$ & & 63.0 & n.a. & n.a. \\
\hline & 126 & - & $\begin{array}{l}\text { Study group post-BFB, pat. } \\
\text { not completed BFB (126) }\end{array}$ & & $(25.0)$ & n.a. & n.a. \\
\hline & 178 & $\begin{array}{l}\text { Study group post-BFB, } \\
\text { pat. completed BFB (52) }\end{array}$ & $\begin{array}{l}\text { Study group post-BFB, pat. } \\
\text { not completed BFB (126) }\end{array}$ & & -38.0 & n.a. & n.a. \\
\hline \multirow[t]{3}{*}{$\begin{array}{l}\text { Parker et al. } \\
2019 \text { [31] }\end{array}$} & 130 & $n / \mathrm{a}$ & $\begin{array}{l}\text { Whole study group post-BFB } \\
\text { (130) }\end{array}$ & \multirow{3}{*}{$\begin{array}{l}\text { SR: \% patients reporting } \\
\text { symptom improvement } \\
\text { ( } \pm \text { improvement in anorec- } \\
\text { tal manometry profile) }\end{array}$} & (55.4) & n.a. & n.a. \\
\hline & 53 & $n / a$ & $\begin{array}{l}\text { Subgroup with constipa- } \\
\text { tion + dys. defecation post- } \\
\text { BFB (53) }\end{array}$ & & $(45.3)$ & n.a. & n.a. \\
\hline & 3 & $n / a$ & $\begin{array}{l}\text { Subgroup with rectal pain } \\
\text { post-BFB (3) }\end{array}$ & & $(0.0)$ & n.a. & n.a. \\
\hline \multicolumn{8}{|c|}{ Male chronic pelvic pain syndrome, Urological Chronic Pelvic Pain Syndrome } \\
\hline \multirow[t]{2}{*}{$\begin{array}{l}\text { Clemens et al. } \\
2000 \text { [32] }\end{array}$} & 16 & Study group pre-BFB (19) & $\begin{array}{l}\text { Study group } 6 \text { months post- } \\
\text { BFB (16) }\end{array}$ & Pain VAS $(0-9)$ & -4 & n.a. & $p=0.001^{b}$ \\
\hline & 16 & Study group pre-BFB (19) & $\begin{array}{l}\text { Study group } 6 \text { months post- } \\
\text { BFB (16) }\end{array}$ & Symptom score: AUA [59] & -7.5 & n.a. & $p=0.001^{b}$ \\
\hline \multirow[t]{2}{*}{$\begin{array}{l}\text { Cornel et al. } \\
2005[34]\end{array}$} & 31 & Pstudy group re-BFB (33) & Study group post-BFB (31) & $\begin{array}{l}\text { Symptom score: NIH-CPSI } \\
\text { subdomain pain }(0-21)\end{array}$ & -5.3 & n.a. & $p=0.001^{b}$ \\
\hline & 31 & Study group pre-BFB (33) & Study group post-BFB (31) & Symptom score: NIH-CPSI & -12.2 & n.a. & $p=0.001^{b}$ \\
\hline
\end{tabular}


Table 5 (Continued)

\begin{tabular}{|c|c|c|c|c|c|c|c|}
\hline Study & $\begin{array}{l}n \text { (total) } \\
\text { (group } 1 \text {, } \\
\text { 2) }\end{array}$ & Group 1 (n1) & Group 2 (n2) & Outcome measure & $\begin{array}{l}\text { Mean difference: } \\
\text { Group } 2 \text { minus } \\
\text { Group } 1[\mathrm{Cl}]\end{array}$ & $\begin{array}{l}\text { Effect size } \\
\text { [strength] }\end{array}$ & $\begin{array}{l}P \text {-value (for } \\
\text { difference in } \\
\text { means) }\end{array}$ \\
\hline \multirow[t]{8}{*}{$\begin{array}{l}\text { Yang et al. } \\
2017 \text { [35] }\end{array}$} & 22 & $\begin{array}{l}\text { IG1 (BFB+EGS) pretreat- } \\
\text { ment (24) }\end{array}$ & $\begin{array}{l}\text { IG1 (BFB + EGS) } 12 \text { weeks } \\
\text { post-treatment (22) }\end{array}$ & \multirow[t]{2}{*}{ Pain VAS $(0-10)$} & $\begin{array}{l}-3.5 \\
{[-4.91 ;-2.09]}\end{array}$ & $-1.74[\mathrm{VL}]$ & $p=0.001^{b}$ \\
\hline & 45 & $\begin{array}{l}\text { IG1 (BFB+EGS) } 12 \text { weeks } \\
\text { post-treatment (22) }\end{array}$ & $\begin{array}{l}\text { IG2 (PEMF) } 12 \text { weeks post- } \\
\text { treatment (23) }\end{array}$ & & $\begin{array}{l}0.6 \\
{[-1.44 ; 0.24]}\end{array}$ & $-0.34[\mathrm{~S}]$ & $p=0.084^{\mathrm{a}}$ \\
\hline & 22 & $\begin{array}{l}\text { IG1 (BFB+EGS) pretreat- } \\
\text { ment (24) }\end{array}$ & $\begin{array}{l}\text { IG1 (BFB + EGS) } 12 \text { weeks } \\
\text { post-treatment (22) }\end{array}$ & \multirow[t]{2}{*}{$\begin{array}{l}\mathrm{NIH}-\mathrm{CPSI} \text { subdomain pain } \\
(0-21)\end{array}$} & $\begin{array}{l}-8.3 \\
{[-10.91 ;-5.70]}\end{array}$ & $-2.23[\mathrm{H}]$ & $p<0.001^{a}$ \\
\hline & 45 & $\begin{array}{l}\text { IG1 (BFB+EGS) } 12 \text { weeks } \\
\text { post-treatment (22) }\end{array}$ & $\begin{array}{l}\text { IG2 (PEMF) } 12 \text { weeks post- } \\
\text { treatment (23) }\end{array}$ & & $\begin{array}{l}0.1 \\
{[-1.98 ; 1.78]}\end{array}$ & -0.03 [VS] & $p=0.035^{\mathrm{a}}$ \\
\hline & 22 & $\begin{array}{l}\text { IG1 (BFB+EGS) pretreat- } \\
\text { ment (24) }\end{array}$ & $\begin{array}{l}\text { IG1 (BFB + EGS) } 12 \text { weeks } \\
\text { post-treatment (22) }\end{array}$ & \multirow[t]{2}{*}{$\begin{array}{l}\text { Symptom score: NIH-CPSI } \\
\text { total score (0-43) }\end{array}$} & $\begin{array}{l}-14.3 \\
{[-19.82 ;-8.78]}\end{array}$ & $-1.81[\mathrm{VL}]$ & $p<0.001^{a}$ \\
\hline & 45 & $\begin{array}{l}\text { IG1 (BFB+EGS) } 12 \text { weeks } \\
\text { post-treatment (22) }\end{array}$ & $\begin{array}{l}\text { IG2 (PEMF) } 12 \text { weeks post- } \\
\text { treatment (23) }\end{array}$ & & $\begin{array}{l}0.5 \\
{[-3.98 ; 2.98]}\end{array}$ & -0.07 [VS] & $p=0.009^{a}$ \\
\hline & 22 & $\begin{array}{l}\text { IG1 (BFB+EGS) pretreat- } \\
\text { ment (24) }\end{array}$ & $\begin{array}{l}\text { IG1 (BFB + EGS) } 12 \text { weeks } \\
\text { post-treatment (22) }\end{array}$ & \multirow[t]{2}{*}{ Symptom score: IPPS } & $\begin{array}{l}-4.6 \\
{[-8.64 ;-0.56]}\end{array}$ & $-0.80[\mathrm{M}]$ & $p=0.004^{a}$ \\
\hline & 45 & $\begin{array}{l}\text { IG1 (BFB+EGS) } 12 \text { weeks } \\
\text { post-treatment (22) }\end{array}$ & $\begin{array}{l}\text { IG2 (PEMF) } 12 \text { weeks post- } \\
\text { treatment (23) }\end{array}$ & & $\begin{array}{l}1.80 \\
{[-3.57 ;-0.03]}\end{array}$ & $-0.49[\mathrm{~S}]$ & $p=0.663^{\mathrm{a}}$ \\
\hline \multirow[t]{2}{*}{$\begin{array}{l}\text { He et al. } 2010 \\
{[36]}\end{array}$} & 21 & Study group pre-BFB (21) & $\begin{array}{l}\text { Study group } 10 \text { weeks post- } \\
\text { BFB (21) }\end{array}$ & $\begin{array}{l}\text { NIH-CPSI subdomain pain } \\
(0-21)\end{array}$ & $\begin{array}{l}-1.80 \\
{[-3.13 ;-0.47]}\end{array}$ & $-0.97[\mathrm{~L}]$ & $p<0.05^{b}$ \\
\hline & 21 & Study group pre-BFB (21) & $\begin{array}{l}\text { Study group } 10 \text { weeks post- } \\
\text { BFB (21) }\end{array}$ & $\begin{array}{l}\text { Symptom score: NIH-CPSI } \\
\text { total score (0-43) }\end{array}$ & $\begin{array}{l}-13.3 \\
{[-16.7 ;-9.92]}\end{array}$ & $-2.83[\mathrm{H}]$ & $p<0.05^{b}$ \\
\hline \multicolumn{8}{|c|}{ Female chronic pelvic pain } \\
\hline \multirow[t]{7}{*}{$\begin{array}{l}\text { Schmitt } 2017 \\
\text { et al. [37] }\end{array}$} & 26 & $\begin{array}{l}\text { Subgroup w pelvic pain/ } \\
\text { dyspareunia pre-treat- } \\
\text { ment (29) }\end{array}$ & $\begin{array}{l}\text { Subgroup w pelvic pain/ } \\
\text { dyspareunia after 3rd treat- } \\
\text { ment (26) }\end{array}$ & \multirow[t]{3}{*}{ Pelvic pain VAS (0-10) } & -1 & n.a. & $p=0.99^{b}$ \\
\hline & 27 & $\begin{array}{l}\text { Subgroup w pelvic pain/ } \\
\text { dyspareunia pre-treat- } \\
\text { ment (29) }\end{array}$ & $\begin{array}{l}\text { Subgroup w pelvic pain/ } \\
\text { dyspareunia after final treat- } \\
\text { ment (27) }\end{array}$ & & -3 & n.a. & $p=0.27^{b}$ \\
\hline & 26 & $\begin{array}{l}\text { Subgroup w pelvic pain/ } \\
\text { dyspareunia after 3rd } \\
\text { treatment (26) }\end{array}$ & $\begin{array}{l}\text { Subgroup w pelvic pain/ } \\
\text { dyspareunia after final treat- } \\
\text { ment (27) }\end{array}$ & & -2 & n.a. & $p=0.02^{b}$ \\
\hline & 27 & $\begin{array}{l}\text { Subgroup w pelvic pain/ } \\
\text { dyspareunia after 3rd } \\
\text { treatment (28) }\end{array}$ & $\begin{array}{l}\text { Subgroup w pelvic pain/ } \\
\text { dyspareunia after final treat- } \\
\text { ment (27) }\end{array}$ & $\begin{array}{l}\text { Rating treatment success } \\
\text { pelvic pain (0: none-10: very } \\
\text { successful) }\end{array}$ & 2 & n.a. & $p=0.51^{b}$ \\
\hline & 14 & $\begin{array}{l}\text { Subgroup w pelvic pain/ } \\
\text { dyspareunia after 3rd } \\
\text { treatment (14) }\end{array}$ & $\begin{array}{l}\text { Subgroup w pelvic pain/ } \\
\text { dyspareunia after final treat- } \\
\text { ment (16) }\end{array}$ & $\begin{array}{l}\text { Rating treatment success } \\
\text { dyspareunia (0: none-10: } \\
\text { very successful) }\end{array}$ & 3 & n.a. & $p=0.20^{b}$ \\
\hline & 79 & $\begin{array}{l}\text { Subgroup w urinary symp- } \\
\text { toms after 3rd treatment } \\
\text { (80) }\end{array}$ & $\begin{array}{l}\text { Subgroup w urinary symp- } \\
\text { toms after final treatment } \\
\text { (79) }\end{array}$ & $\begin{array}{l}\text { Rating treatment success uri- } \\
\text { nary symptoms (0: none-10: } \\
\text { very successful) }\end{array}$ & 2 & n.a. & $p<0.001^{b}$ \\
\hline & 15 & $\begin{array}{l}\text { Subgroup w defecatory } \\
\text { symptoms after 3rd treat- } \\
\text { ment (16) }\end{array}$ & $\begin{array}{l}\text { Subgroup w defecatory symp- } \\
\text { toms after final treatment } \\
\text { (15) }\end{array}$ & $\begin{array}{l}\text { Rating treatment success } \\
\text { defecatory symptoms (0: } \\
\text { none-10: very successful) }\end{array}$ & 1 & n.a. & $p=0.003^{b}$ \\
\hline \multirow[t]{3}{*}{$\begin{array}{l}\text { Glazer et al. } \\
1995[38]\end{array}$} & 33 & Study group pre-BFB (33) & $\begin{array}{l}\text { Study group after } 6 \text { th clinical } \\
\text { reevaluation with BFB (33) }\end{array}$ & \multirow[t]{2}{*}{ Pelvic pain VAS $(0-10)$} & -5.7 & n.a. & $p<0.001^{b}$ \\
\hline & 33 & Study group pre-BFB (33) & $\begin{array}{l}\text { Study group } 6 \text { months after } \\
6 \text { th clinical reevaluation (33) }\end{array}$ & & -6 & n.a. & $p<0.001^{b}$ \\
\hline & 33 & Study group pre-BFB (33) & $\begin{array}{l}\text { Study group after } 6 \text { th clinical } \\
\text { reevaluation }+6 \text { months later } \\
\text { (33) }\end{array}$ & $\begin{array}{l}\text { SR: } \% \text { of patients reporting } \\
\text { intercrouse } \geq 1 / \text { month }\end{array}$ & 66.67 & n.a. & $p<0.001^{b}$ \\
\hline \multirow[t]{2}{*}{$\begin{array}{l}\text { McKay et al. } \\
2001 \text { [39] }\end{array}$} & 11 & $\begin{array}{l}\text { Study group } 1 \text { month } \\
\text { post-BFB (19) }\end{array}$ & $\begin{array}{l}\text { Study group } 6 \text { months post } \\
\text { BFB (11) }\end{array}$ & Pelvic pain VAS $(0-10)$ & -6.8 & n.a. & n.a. \\
\hline & 11 & $\begin{array}{l}\text { Study group } 1 \text { month } \\
\text { post-BFB (19) }\end{array}$ & $\begin{array}{l}\text { Study group } 6 \text { months post } \\
\text { BFB (11) }\end{array}$ & $\begin{array}{l}\text { SR: \% patients reporting } \\
\text { intercourse }\end{array}$ & 88.9 & n.a. & n.a. \\
\hline
\end{tabular}




\section{review article}

Table 5 (Continued)

\begin{tabular}{|c|c|c|c|c|c|c|c|}
\hline Study & $\begin{array}{l}n \text { (total) } \\
\text { (group 1, } \\
\text { 2) }\end{array}$ & Group 1 (n1) & Group 2 (n2) & Outcome measure & $\begin{array}{l}\text { Mean difference: } \\
\text { Group } 2 \text { minus } \\
\text { Group } 1[\mathrm{Cl}]\end{array}$ & $\begin{array}{l}\text { Effect size } \\
\text { [strength] }\end{array}$ & $\begin{array}{l}P \text {-value (for } \\
\text { difference in } \\
\text { means) }\end{array}$ \\
\hline \multirow[t]{9}{*}{$\begin{array}{l}\text { Gentilcore- } \\
\text { Saulnier et al. } \\
2010 \text { [40] }\end{array}$} & 11 & $\begin{array}{l}\text { IG pre-BFB in pat. with } \\
\text { provoked vestibulodynia } \\
\text { (11) }\end{array}$ & $\begin{array}{l}\text { IG post-BFB in pat. w pro- } \\
\text { voked vestibulodynia (11) }\end{array}$ & \multirow[t]{3}{*}{$\begin{array}{l}\text { Pain NRS }(0-10) \text { during digital } \\
\text { intravaginal assessment of } \\
\text { superficial + deep PF }\end{array}$} & $\begin{array}{l}-2.00 \\
{[-3.33 ;-0.67]}\end{array}$ & $-1.56[\mathrm{VL}]$ & $p=0.007^{a}$ \\
\hline & 22 & $\begin{array}{l}\text { IG pre-BFB in pat. w } \\
\text { provoked vestibulodynia } \\
\text { (11) }\end{array}$ & $\begin{array}{l}\text { Healthy CG without interven- } \\
\text { tion (11) }\end{array}$ & & $\begin{array}{l}-2.13 \\
{[-1.27 ;-2.99]}\end{array}$ & $-1.73[\mathrm{VL}]$ & $p=0.002^{a}$ \\
\hline & 22 & $\begin{array}{l}\text { IG post-BFB in pat. w } \\
\text { provoked vestibulodynia } \\
\text { (11) }\end{array}$ & $\begin{array}{l}\text { Healthy CG without interven- } \\
\text { tion (11) }\end{array}$ & & $\begin{array}{l}-0.13 \\
{[-0.26 ; 0.52]}\end{array}$ & $0.23[S]$ & $p=0.58^{\mathrm{a}}$ \\
\hline & 11 & $\begin{array}{l}\text { IG pre-BFB in pat. w } \\
\text { provoked vestibulodynia } \\
\text { (11) }\end{array}$ & $\begin{array}{l}\text { IG post-BFB in pat. w pro- } \\
\text { voked vestibulodynia (11) }\end{array}$ & \multirow[t]{3}{*}{$\begin{array}{l}\text { "unpleasantness" NRS (0-10) } \\
\text { during manual assessment }\end{array}$} & $\begin{array}{l}-1.63 \\
{[-3.03 ;-023]}\end{array}$ & $-1.21[\mathrm{VL}]$ & $\begin{array}{l}p= \\
0.0009^{a}\end{array}$ \\
\hline & 22 & $\begin{array}{l}\text { IG pre-BFB in pat. w } \\
\text { provoked vestibulodynia } \\
\text { (11) }\end{array}$ & $\begin{array}{l}\text { Healthy CG without interven- } \\
\text { tion (11) }\end{array}$ & & $\begin{array}{l}-0.54 \\
{[-0.50 ; 1.58]}\end{array}$ & $0.36[S]$ & $p=0.40^{\mathrm{a}}$ \\
\hline & 22 & $\begin{array}{l}\text { IG post-BFB in pat. w } \\
\text { provoked vestibulodynia } \\
\text { (11) }\end{array}$ & $\begin{array}{l}\text { Healthy CG without interven- } \\
\text { tion (11) }\end{array}$ & & $\begin{array}{l}-1.09 \\
{[-2.01 ;-0.17]}\end{array}$ & $-0.83[\mathrm{~L}]$ & $p=0.07^{\mathrm{a}}$ \\
\hline & 11 & $\begin{array}{l}\text { IG pre-BFB in pat. w } \\
\text { provoked vestibulodynia } \\
\text { (11) }\end{array}$ & $\begin{array}{l}\text { IG post-BFB in pat. w pro- } \\
\text { voked vestibulodynia (11) }\end{array}$ & \multirow[t]{3}{*}{$\begin{array}{l}\text { Painful pressure stimulus } \\
\text { intensity levels (pressure to } \\
\text { induce pain NRS } 6 / 10, \mathrm{~g} / \mathrm{cm}^{2} \text { ) }\end{array}$} & $\begin{array}{l}92.00 \\
{[-162.25} \\
346.25]\end{array}$ & $0.37[S]$ & $p=0.07^{\mathrm{a}}$ \\
\hline & 22 & $\begin{array}{l}\text { IG pre-BFB in pat. w } \\
\text { provoked vestibulodynia } \\
\text { (11) }\end{array}$ & $\begin{array}{l}\text { Healthy CG without interven- } \\
\text { tion (11) }\end{array}$ & & $\begin{array}{l}201.00 \\
{[-366.64} \\
-35.36]\end{array}$ & $-0.85[\mathrm{~L}]$ & $p=0.001^{a}$ \\
\hline & 22 & $\begin{array}{l}\text { IG post-BFB in pat. w } \\
\text { provoked vestibulodynia } \\
\text { (11) }\end{array}$ & $\begin{array}{l}\text { Healthy CG without interven- } \\
\text { tion (11) }\end{array}$ & & $\begin{array}{l}109.00 \\
{[-246.63 ; 28.63]}\end{array}$ & $-0.56[\mathrm{M}]$ & $p=0.03^{a}$ \\
\hline \multirow[t]{3}{*}{$\begin{array}{l}\text { Bendana et } \\
\text { al. } 2009 \text { [41] }\end{array}$} & 21 & Study group pre-BFB (52) & $\begin{array}{l}\text { Study group } 3 \text { months post- } \\
\text { BFB (21) }\end{array}$ & $\begin{array}{l}\text { AUA symptom score-total } \\
\text { score }(0-35)\end{array}$ & $\begin{array}{l}-7.97 \\
{[-12.25 ;-4.62]}\end{array}$ & $-1.10[\mathrm{~L}]$ & $p<0.001^{a}$ \\
\hline & 21 & Study group pre-BFB (52) & $\begin{array}{l}\text { Study group } 3 \text { months post- } \\
\text { BFB (21) }\end{array}$ & $\begin{array}{l}\text { American Urological Associa- } \\
\text { tion bother score }(0-6)\end{array}$ & $\begin{array}{l}-1.53 \\
{[-2.33 ;-0.87]}\end{array}$ & $-1.13[\mathrm{~L}]$ & $p<0.001^{a}$ \\
\hline & 13 & Study group pre-BFB (52) & $\begin{array}{l}\text { Study group } 3 \text { months post- } \\
\text { BFB (13) }\end{array}$ & $\begin{array}{l}\text { VAS for symptom severity } \\
(1=\text { lowest } 10=\text { most severe })\end{array}$ & $\begin{array}{l}-2.44 \\
\text { [n.a.] }\end{array}$ & n.a. & $p<0.001^{a}$ \\
\hline \multirow[t]{2}{*}{$\begin{array}{l}\text { Philips et al. } \\
1992 \text { [42] }\end{array}$} & 10 & $\begin{array}{l}\text { IG1 (BFB group) pre-BFB } \\
\text { (10) }\end{array}$ & $\begin{array}{l}\text { IG1 (BFB group) post-BFB } \\
\text { (10) }\end{array}$ & \multirow[t]{2}{*}{ Pain score: self-monitored } & $\begin{array}{l}-1.30 \\
{[-29.40 ; 26.80]}\end{array}$ & -0.05 [VS] & n.a. \\
\hline & 10 & $\begin{array}{l}\text { IG1 (BFB group) pre-BFB } \\
\text { (10) }\end{array}$ & $\begin{array}{l}\text { IG1 (BFB group) } 2 \text { months } \\
\text { post-BFB (10) }\end{array}$ & & $\begin{array}{l}-11.40 \\
{[-26.55 ; 3.75]}\end{array}$ & $-0.82[\mathrm{~L}]$ & n.a. \\
\hline \multirow[t]{4}{*}{$\begin{array}{l}\text { Hart et al. } \\
1981[43]\end{array}$} & 5 & $\begin{array}{l}\text { IG1: EMG BFB (5) } \\
\text { baseline }\end{array}$ & $\begin{array}{l}\text { IG1: EMG BFB (5) posttreat- } \\
\text { ment }\end{array}$ & \multirow{4}{*}{$\begin{array}{l}\text { Symptom score: SSS total } \\
\text { score: total of } 15 \text { symptoms } \\
\text { ( } 1 \text { best }-5 \text { worst) }\end{array}$} & $\begin{array}{l}-7.00 \\
{[-11.85 ;-2.15]}\end{array}$ & $-2.51[\mathrm{H}]$ & n.a. \\
\hline & 5 & $\begin{array}{l}\text { IG1: EMG BFB (5) } \\
\text { baseline }\end{array}$ & $\begin{array}{l}\text { IG1: EMG BFB (5) } 8 \text { weeks } \\
\text { posttreatment }\end{array}$ & & $\begin{array}{l}-9.30 \\
{[-12.61 ;-6.00]}\end{array}$ & $-4.89[\mathrm{H}]$ & n.a. \\
\hline & 6 & $\begin{array}{l}\text { IG2: BFB skin tempera- } \\
\text { ture baseline (6) }\end{array}$ & $\begin{array}{l}\text { IG2: BFB skin tempera- } \\
\text { ture posttreatment (6) }\end{array}$ & & $\begin{array}{l}-2.00 \\
{[-8.86 ; 4.86]}\end{array}$ & $-0.44[S]$ & n.a. \\
\hline & 6 & $\begin{array}{l}\text { IG2: BFB skin temperature } \\
\text { baseline (6) }\end{array}$ & $\begin{array}{l}\text { IG2: BFB skin temperature } 8 \\
\text { weeks posttreatment (6) }\end{array}$ & & $\begin{array}{l}-8.10 \\
{[-14.25 ;-1.95]}\end{array}$ & $-2.00[\mathrm{H}]$ & n.a. \\
\hline \multirow[t]{3}{*}{$\begin{array}{l}\text { Bennink et al. } \\
1982[44]\end{array}$} & 5 & $\begin{array}{l}\text { IG1 (relaxation + EMG } \\
\text { BFB) pretreatment (5) }\end{array}$ & $\begin{array}{l}\text { IG1 (relaxation+EMG BFB) } \\
\text { posttreatment (5) }\end{array}$ & $\begin{array}{l}\text { Symptom score: SSS total } \\
\text { of } 15 \text { symptoms ( } 1 \text { best }-5 \\
\text { worst) }\end{array}$ & $\begin{array}{l}-3.6 \\
{[-14.27 ; 7.07]}\end{array}$ & $-0.59[\mathrm{M}]$ & n.a. \\
\hline & 5 & $\begin{array}{l}\text { IG1 (relaxation+EMG } \\
\text { BFB) pretreatment (5) }\end{array}$ & $\begin{array}{l}\text { IG1 (relaxation + EMG BFB) } \\
\text { posttreatment (5) }\end{array}$ & $\begin{array}{l}\text { SSS of subdomain cramps, } \\
\text { backache, abdominal pain } \\
\text { (1-5= very severely) }\end{array}$ & $\begin{array}{l}-1.6 \\
{[-4.63 ; 1.43]}\end{array}$ & $-0.92[\mathrm{~L}]$ & n.a. \\
\hline & 5 & $\begin{array}{l}\text { IG1 (relaxation + EMG } \\
\text { BFB) pretreatment (5) }\end{array}$ & $\begin{array}{l}\text { IG1 (relaxation+EMG BFB) } \\
\text { posttreatment (5) }\end{array}$ & $\begin{array}{l}\text { SSS of subdomain cramps } \\
\text { only (1-5= very severely) }\end{array}$ & $\begin{array}{l}-0.6 \\
{[-1.47 ; 0.27]}\end{array}$ & $-1.19[\mathrm{~L}]$ & n.a. \\
\hline
\end{tabular}


Table 5 (Continued)

\begin{tabular}{|c|c|c|c|c|c|c|c|}
\hline Study & $\begin{array}{l}n \text { (total) } \\
\text { (group 1, } \\
\text { 2) }\end{array}$ & Group 1 (n1) & Group 2 (n2) & Outcome measure & $\begin{array}{l}\text { Mean difference: } \\
\text { Group } 2 \text { minus } \\
\text { Group } 1[\mathrm{Cl}]\end{array}$ & $\begin{array}{l}\text { Effect size } \\
\text { [strength] }\end{array}$ & $\begin{array}{l}P \text {-value (for } \\
\text { difference in } \\
\text { means) }\end{array}$ \\
\hline \multirow[t]{6}{*}{$\begin{array}{l}\text { Vagedes et al. } \\
2019 \text { [45] }\end{array}$} & 20 & $\begin{array}{l}\text { IG1 (BFB group) pre-BFB } \\
\text { (20) }\end{array}$ & $\begin{array}{l}\text { IG1 (BFB group) post-BFB } \\
(20)\end{array}$ & \multirow[t]{3}{*}{$\begin{array}{l}\text { Mean NRS }(0-10) \text { pain during } \\
\text { menstruation }\end{array}$} & $\begin{array}{l}-0.3 \\
{[-1.2 / 0.6]^{9}}\end{array}$ & $-0.2[\mathrm{VS}]^{\mathrm{g}}$ & n.a. \\
\hline & 37 & $\begin{array}{l}\text { IG1 (BFB group) postBFB } \\
(20)\end{array}$ & No treatment CG (17) & & $\begin{array}{l}0.9 \\
{[-2.10 / 0.30]^{g}}\end{array}$ & $-0.51[\mathrm{M}]^{\mathrm{g}}$ & $p=0.211$ \\
\hline & 43 & $\begin{array}{l}\text { IG1 (BFB group) postBFB } \\
\text { (20) }\end{array}$ & $\begin{array}{l}\text { IG2 (rhythmical massage) } \\
\text { post treatment (23) }\end{array}$ & & $\begin{array}{l}-0.6 \\
{[-1.82 / 0.40]^{9}}\end{array}$ & $-0.34[S]^{g}$ & $p=0.361$ \\
\hline & 20 & $\begin{array}{l}\text { IG1 (BFB group) pre-BFB } \\
\text { (20) }\end{array}$ & $\begin{array}{l}\text { IG1 (BFB group) post-BFB } \\
\text { (20) }\end{array}$ & \multirow[t]{3}{*}{$\begin{array}{l}\text { Maximum NRS (0-10) pain } \\
\text { during menstruation }\end{array}$} & $\begin{array}{l}-0.5 \\
{[-1.4 / 0.3]^{9}}\end{array}$ & $-0.2[S]^{9}$ & n.a. \\
\hline & 37 & $\begin{array}{l}\text { IG1 (BFB group) post-BFB } \\
\text { (20) }\end{array}$ & No-treatment CG (17) & & $\begin{array}{l}0.6 \\
{[-2.18 / 0.74]^{g}}\end{array}$ & $-0.40[S]^{g}$ & $p>0.05$ \\
\hline & 43 & $\begin{array}{l}\text { IG1 (BFB group) post-BFB } \\
\text { (20) }\end{array}$ & $\begin{array}{l}\text { IG2 (rhythmical massage) } \\
\text { post treatment (23) }\end{array}$ & & $\begin{array}{l}-0.6 \\
{[-1.94 / 0.76]^{9}}\end{array}$ & $-0.23[\mathrm{~S}]^{\mathrm{g}}$ & $p>0.05$ \\
\hline \multirow[t]{3}{*}{$\begin{array}{l}\text { Starr et al. } \\
2013 \text { [46] }\end{array}$} & 694 & $\begin{array}{l}\text { Pre-BFB in pat. w urinary } \\
\text { symptoms (694) }\end{array}$ & $\begin{array}{l}\text { Post-BFB in pat. w urinary } \\
\text { symptoms (n.a.) }\end{array}$ & $\begin{array}{l}\% \text { subjective global urinary } \\
\text { symptom improvement since } \\
\text { initial session (0: none-100\%: } \\
\text { perfect) }\end{array}$ & $\begin{array}{l}\text { Mean } 80-85 \% \\
\text { improvement }^{h}\end{array}$ & n.a. & n.a. \\
\hline & 187 & $\begin{array}{l}\text { Pre-BFB in pat. w bowl } \\
\text { symptoms (187) }\end{array}$ & $\begin{array}{l}\text { Post-BFB in pat. w bowl } \\
\text { symptoms (n.a.) }\end{array}$ & $\begin{array}{l}\text { \% subjective global bowel } \\
\text { symptom improvement since } \\
\text { initial session (0: none-100\%: } \\
\text { perfect) }\end{array}$ & $\begin{array}{l}\text { Mean } 80-85 \% \\
\text { improvement }^{h}\end{array}$ & n.a. & n.a. \\
\hline & 368 & $\begin{array}{l}\text { Pre-BFB in pat. w pelvic } \\
\text { pain symptoms (368) }\end{array}$ & $\begin{array}{l}\text { Post-BFB in pat. w pelvic } \\
\text { pain symptoms (n.a.) }\end{array}$ & $\begin{array}{l}\% \text { subjective global pelvic } \\
\text { pain symptom improvement } \\
\text { since the initial session ( } 0 \text { : } \\
\text { none- } 100 \% \text { : perfect) }\end{array}$ & $\begin{array}{l}\text { Mean } 50-90 \% \\
\text { improve-ment }^{\mathrm{h}}\end{array}$ & n.a. & $p>0.05$ \\
\hline \multirow[t]{2}{*}{$\begin{array}{l}\text { Lúcio et al. } \\
2014 \text { [47] }\end{array}$} & 6 & $\begin{array}{l}\text { IG1 pre BFB, PFM train- } \\
\text { ing and sham-electro- } \\
\text { stimulation (6) }\end{array}$ & $\begin{array}{l}\text { IG1 post BFB, PFM training } \\
\text { and sham electrostimulation } \\
\text { (6) }\end{array}$ & $\begin{array}{l}\text { Symptom score: FSFI subdo- } \\
\text { main pain }\end{array}$ & 1.6 & n.a. & $p>0.05^{b}$ \\
\hline & 6 & $\begin{array}{l}\text { IG1 pre BFB, PFM train- } \\
\text { ing and sham-electro- } \\
\text { stimulation (6) }\end{array}$ & $\begin{array}{l}\text { IG1 post BFB, PFM training } \\
\text { and sham-electro-stimulation } \\
\text { (6) }\end{array}$ & $\begin{array}{l}\text { Symptom score: FSFI total } \\
\text { score }(2.0-36.0=\text { best })\end{array}$ & -10 & n.a. & $p<0.05^{b}$ \\
\hline \multirow[t]{4}{*}{$\begin{array}{l}\text { Aalaie et al. } \\
2020 \text { [48] }\end{array}$} & 9 & $\begin{array}{l}\text { IG1 (BFB group) pre- } \\
\text { treatment (10) }\end{array}$ & $\begin{array}{l}\text { IG1 (BFB group) } 3 \text { months } \\
\text { post-treatment (9) }\end{array}$ & $\begin{array}{l}\text { Symptom score: FSFI subdo- } \\
\text { main pain }\end{array}$ & $\begin{array}{l}0.9 \\
{[0.1 ; 1.6]^{g}}\end{array}$ & $\begin{array}{l}\eta 2=0.66 \\
{[L]^{g}}\end{array}$ & $p=0.026$ \\
\hline & 20 & $\begin{array}{l}\text { IG1 (BFB group) } 3 \text { months } \\
\text { post-treatment (9) }\end{array}$ & $\begin{array}{l}\text { IG2 (EGS) } 3 \text { months post- } \\
\text { treatment (11) }\end{array}$ & - & n.a. & $\begin{array}{l}\eta 2=0.01 \\
{[S]^{g}}\end{array}$ & $p=0.985$ \\
\hline & 9 & $\begin{array}{l}\text { IG1 (BFB group) pre- } \\
\text { treatment (10) }\end{array}$ & $\begin{array}{l}\text { IG1 (BFB group) } 3 \text { months } \\
\text { post-treatment (9) }\end{array}$ & \multirow[t]{2}{*}{$\begin{array}{l}\text { Symptom score: FSFI total } \\
\text { score }(2.0-36.0=\text { best })\end{array}$} & $\begin{array}{l}8.9 \\
{[7.0 ; 10.9]^{9}}\end{array}$ & $\begin{array}{l}\eta 2=0.96 \\
{[\mathrm{~L}]^{9}}\end{array}$ & $p<0.001$ \\
\hline & 20 & $\begin{array}{l}\text { IG1 (BFB group) } 3 \text { months } \\
\text { post-treatment (9) }\end{array}$ & $\begin{array}{l}\text { IG2 (EGS) } 3 \text { months post- } \\
\text { treatment (11) }\end{array}$ & & n.a. & $\begin{array}{l}\eta 2=0.64 \\
{[L]^{g}}\end{array}$ & $p=0.002$ \\
\hline \multicolumn{8}{|c|}{ Chronic pelvic pain in children } \\
\hline \multirow{2}{*}{$\begin{array}{l}\text { Hoebeke et } \\
\text { al. } 2004 \text { [51] }\end{array}$} & 21 & $n / a$ & Study group post BFB (21) & \multirow{2}{*}{$\begin{array}{l}\text { SR: \% patients reporting } \\
\text { complete pain relief }\end{array}$} & 80.95 & n.a. & n.a. \\
\hline & 21 & $n / a$ & $\begin{array}{l}\text { Study group long-term f/u } \\
\text { (16 months) (21) }\end{array}$ & & 66.67 & n.a. & n.a. \\
\hline \multirow[t]{4}{*}{$\begin{array}{l}\text { Ebiloglu et al. } \\
2016 \text { [52] }\end{array}$} & 136 & $\begin{array}{l}\text { Whole study group pre- } \\
\text { BFB [136] }\end{array}$ & $\begin{array}{l}\text { Whole study group post BFB } \\
\text { (6 months) (136) }\end{array}$ & \multirow[t]{3}{*}{ SR: \% patients with dysuria } & -19.85 & n.a. & $p=0.007^{c}$ \\
\hline & 107 & $\begin{array}{l}\text { Subgroup OBS and dysf. } \\
\text { voiding pre-BFB (107) }\end{array}$ & $\begin{array}{l}\text { Post BFB ( } 6 \text { months) in this } \\
\text { subgroup (107) }\end{array}$ & & -20.56 & n.a. & $p<0.001^{c}$ \\
\hline & 29 & $\begin{array}{l}\text { Subgroup OBS only pre- } \\
\text { BFB (29) }\end{array}$ & $\begin{array}{l}\text { Post BFB ( } 6 \text { months) in this } \\
\text { subgroup ( } 29 \text { ) }\end{array}$ & & -17.24 & n.a. & $p<0.001^{c}$ \\
\hline & 136 & $\begin{array}{l}\text { Whole study group pre- } \\
\text { BFB (136) }\end{array}$ & $\begin{array}{l}\text { Whole study group post BFB } \\
\text { (6 months) (136) }\end{array}$ & Symptom score: LUTDSS & -8.2 & n.a. & $p<0.001^{c}$ \\
\hline \multirow[t]{2}{*}{$\begin{array}{l}\text { Ergin et al. } \\
2016 \text { [53] }\end{array}$} & 39 & $\begin{array}{l}\text { IG pat. w dysfunctional } \\
\text { voiding pre-BFB in (52) }\end{array}$ & IG post BFB (6th month) (39) & SR: \% patients with dysuria & -83.3 & n.a. & $p=0.063^{c}$ \\
\hline & 39 & $\begin{array}{l}\text { IG pat. w dysfunctional } \\
\text { voiding pre-BFB (52) }\end{array}$ & IG post BFB (6th month) (39) & Symptom score: DVISSS & -8.3 & n.a. & $p=0.019$ \\
\hline \multirow[t]{2}{*}{$\begin{array}{l}\text { Li et al. } 2006 \\
\text { [54] }\end{array}$} & 25 & $\begin{array}{l}\text { IG pat. w chronic prostati- } \\
\text { tis pre-BFB (25) }\end{array}$ & $\begin{array}{l}\text { IG post BFB (after 12 } \\
\text { weeks) (25) }\end{array}$ & $\begin{array}{l}\text { Symptom score: NIH-CPSI } \\
\text { subdomain pain }(0-21)\end{array}$ & -2 & n.a. & $p=0.001^{b}$ \\
\hline & 25 & $\begin{array}{l}\text { IG pat. w chronic prostati- } \\
\text { tis pre-BFB (25) }\end{array}$ & $\begin{array}{l}\text { IG post BFB (after } \sim 12 \\
\text { weeks) (25) }\end{array}$ & $\begin{array}{l}\text { Symptom score: NIH-CPSI } \\
\text { total score (0-43) }\end{array}$ & -17 & n.a. & $p<0.001^{b}$ \\
\hline
\end{tabular}




\begin{tabular}{|c|c|c|c|c|c|c|c|}
\hline Study & $\begin{array}{l}n \text { (total) } \\
\text { (group 1, } \\
\text { 2) }\end{array}$ & Group 1 (n1) & Group 2 (n2) & Outcome measure & $\begin{array}{l}\text { Mean difference: } \\
\text { Group } 2 \text { minus } \\
\text { Group } 1[\mathrm{Cl}]\end{array}$ & $\begin{array}{l}\text { Effect size } \\
\text { [strength] }\end{array}$ & $\begin{array}{l}P \text {-value (for } \\
\text { difference in } \\
\text { means) }\end{array}$ \\
\hline \multicolumn{8}{|c|}{ Musculoskeletal, low back pain, myofascial pain } \\
\hline \multirow[t]{2}{*}{$\begin{array}{l}\text { Kent et al. } \\
2015 \text { [55] }\end{array}$} & 58 & IG pre-BFB (58) & IG 3 months post-BFB (58) & \multirow[t]{2}{*}{ Pain VAS $(0-10)$} & $\begin{array}{l}-20.5 \\
{[-30.45 ;-10.55]}\end{array}$ & $-0.87[\mathrm{VL}]$ & n.a. \\
\hline & 54 & $\begin{array}{l}\text { IG pre-Guidelines Care } \\
\text { (54) }\end{array}$ & $\begin{array}{l}\text { IG } 3 \text { months post-Guidelines } \\
\text { care (54) }\end{array}$ & & $\begin{array}{l}-6.5 \\
{[-9.34 ;-3.61]}\end{array}$ & $-0.98[\mathrm{VL}]$ & n.a. \\
\hline \multicolumn{8}{|c|}{ 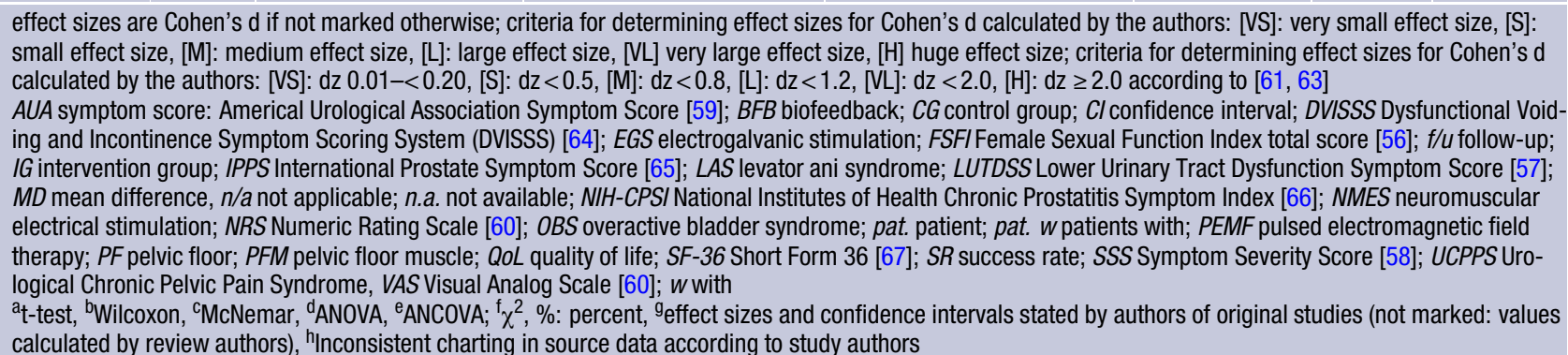 } \\
\hline
\end{tabular}

quality of life postintervention in eight trials (Table 6). The findings came along with small $[29,35]$ to huge [26, 36] effect sizes, with 5 studies showing a significant effect for at least some outcomes [26, 29, 35, $36,45]$. A home-based heart rate variability training failed to significantly improve quality of life compared to standard care [45].

\section{Effect of biofeedback interventions on physiological parameters}

Table 7 presents significant changes in physiological outcome assessment following biofeedback interventions. Biofeedback training could significantly improve at least some manometric values in 9 [16, 20-22, $24-26,28,31]$ out of 10 studies on anorectal dysfunction. In Heah et al. [17] manometric values did not significantly change posttreatment. Six [16, 17, 21, 22, $24,28]$ out of 9 studies did not improve resting anal canal pressures. Studies on constipation and dyssynergic defecation found that paradoxical contraction on evacuation $[21,22,24,28,31]$ and the ability to defecate a balloon [21, 28, 31] could be improved. Patients with dyssynergic defecation could improve more manometric values than patients with slow transit constipation [28]. A landmark trial on anal pain syndrome showed that patients with a tenderness of the levator ani muscle on digital palpation could improve more manometric values than patients without tenderness on the rectal examination [16].

In female chronic pelvic pain, four studies did improve EMG values of the pelvic floor or lower abdomen [38, 39, 44, 52] whereas two (mostly) failed to do so $[40,42]$.

In urologic phenotypes all $[36,53]$ or some [52, 54] urodynamic measures could be significantly improved.

\section{Discussion}

\section{Quality}

This systematic review included 37 quantitative studies and found tentative evidence that biofeedback-assisted training interventions can improve the primarily evaluated outcomes pain, overall symptoms, and quality of life. Results should be considered with caution due to quality issues of many of the included trials. Only 9 studies had an RCT design, out of which 7 were judged to be of good quality according to PEDro assessment. Many studies were likely underpowered and did not provide a sample size calculation.

Biofeedback is a modality to improve self-efficacy and learning based on operant conditioning [83]. Biofeedback is not used as an intervention on its own but is rather an adjunctive tool to other standard interventions (e.g. pelvic floor exercises, education, lifestyle modification [84]). At times, studies applied biofeedback together with additional physical modalities. Besides, patients were often under medication during the study period for symptom control. Therefore, the single effect of biofeedback intervention is difficult to extract. Biofeedback protocols are difficult to compare between institutions as treatment protocols, biofeedback devices and training amount varied considerably.

Most studies compare improvements within an intervention group which reduces the strength of evidence. Most authors drew their conclusions based on the statistical significance, only two papers $[45,48]$ reported on effect sizes and confidence intervals of pain and symptom outcomes. Ten studies [21, 26, 29, 35, 36, 40-44] provided data to calculate effect sizes and confidence intervals to evaluate the clinical relevance of the results [85]. The majority of the studies did not perform a post hoc analysis or a correction 
Table 6 Primary outcome: effect of biofeedback interventions on quality of life

\begin{tabular}{|c|c|c|c|c|c|c|c|c|c|c|}
\hline Study & $\begin{array}{l}n \text { (total } \\
\text { group 1, } \\
\text { 2) }\end{array}$ & Group 1 (n1) & Group 2 (n2) & Outcome measure & $\begin{array}{l}\text { MD: group } 2 \\
\text { minus } \\
\text { group } 1\end{array}$ & $\begin{array}{l}95 \% \mathrm{Cl} \\
\text { lower } \\
\text { bound }\end{array}$ & $\begin{array}{l}95 \% \mathrm{Cl} \\
\text { upper } \\
\text { bound }\end{array}$ & \multicolumn{2}{|c|}{$\begin{array}{l}\text { Effect size } \\
\text { [strength] }\end{array}$} & $\begin{array}{l}P \text {-value (for } \\
\text { difference in } \\
\text { means) }\end{array}$ \\
\hline \multicolumn{11}{|c|}{ Constipation, dyssynergic defecation } \\
\hline \multirow{3}{*}{$\begin{array}{l}\text { Ba-Bai-Ke- } \\
\text { Re et al. } 2014 \\
\text { [26] }\end{array}$} & 88 & IG1 (BFB) (44) & $\begin{array}{l}\text { IG2 (laxatives) } \\
\text { (44) }\end{array}$ & $\begin{array}{l}\text { PAC-QoL } 1 \text { month } \\
\text { post }\end{array}$ & 12.00 & 10.904 & 13.096 & 3.731 & $\mathrm{H}$ & $p<0.001^{a}$ \\
\hline & 88 & IG1 (BFB) (44) & $\begin{array}{l}\text { IG2 (laxatives) } \\
\text { (44) }\end{array}$ & $\begin{array}{l}\text { PAC-QoL } 3 \text { months } \\
\text { post }\end{array}$ & 14.00 & 13.077 & 14.923 & 5.173 & $\mathrm{H}$ & $p<0.001^{a}$ \\
\hline & 88 & IG1 (BFB) (44) & $\begin{array}{l}\text { IG2 (laxatives) } \\
\text { (44) }\end{array}$ & $\begin{array}{l}\text { PAC-QoL } 6 \text { months } \\
\text { post }\end{array}$ & 16.00 & 15.299 & 16.701 & 7.784 & $\mathrm{H}$ & $p<0.001^{a}$ \\
\hline \multirow[t]{13}{*}{$\begin{array}{l}\text { Zhu et al. } \\
2011 \text { [29] }\end{array}$} & 36 & $\begin{array}{l}\text { Study group pre-BFB } \\
\text { (36) }\end{array}$ & $\begin{array}{l}\text { Study group } \\
\text { post-BFB (36) }\end{array}$ & $\begin{array}{l}\text { SF-36: physical } \\
\text { functioning }\end{array}$ & 7.30 & -0.679 & 15.279 & 0.494 & S & $p=0.001^{a}$ \\
\hline & 36 & Pre-BFB (36) & Post-BFB (36) & SF-36: role physical & 23.80 & 2.347 & 45.253 & 0.599 & M & $p<0.001^{a}$ \\
\hline & 36 & Pre-BFB (36) & Post-BFB (36) & SF-36: bodily pain & 10.30 & -1.301 & 21.901 & 0.479 & S & $p=0.001^{a}$ \\
\hline & 36 & Pre-BFB (36) & Post-BFB (36) & SF-36: vitality & 8.00 & -3.932 & 19.932 & 0.362 & S & $p=0.042^{a}$ \\
\hline & 36 & Pre-BFB (36) & Post-BFB (36) & $\begin{array}{l}\text { SF-36: role emo- } \\
\text { tional }\end{array}$ & 19.50 & 0.387 & 38.613 & 0.551 & M & $p=0.001^{a}$ \\
\hline & 36 & Pre-BFB (36) & Post-BFB (36) & SF-36: mental health & 11.00 & 0.368 & 21.632 & 0.559 & M & $p=0.003^{a}$ \\
\hline & 36 & Pre-BFB (36) & Post-BFB (36) & $\begin{array}{l}\text { SF-36: social func- } \\
\text { tion }\end{array}$ & 10.90 & -0.701 & 22.510 & 0.507 & M & $p=0.014^{a}$ \\
\hline & 36 & Pre-BFB (36) & Post-BFB (36) & $\begin{array}{l}\text { SF-36: general } \\
\text { health }\end{array}$ & 10.50 & -1.705 & 22.704 & 0.465 & S & $p=0.008^{a}$ \\
\hline & 36 & Pre-BFB (36) & Post-BFB (36) & $\begin{array}{l}\text { PAC-Q0L: physical } \\
\text { discomfort }\end{array}$ & -0.99 & -1.561 & -0.419 & -0.937 & L & $p<0.001^{a}$ \\
\hline & 36 & Pre-BFB (36) & Post-BFB (36) & $\begin{array}{l}\text { PAC-Q0L: psycho- } \\
\text { social discomfort }\end{array}$ & -0.37 & -0.784 & 0.044 & -0.482 & S & $p<0.001^{a}$ \\
\hline & 36 & Pre-BFB (36) & Post-BFB (36) & $\begin{array}{l}\text { PAC-QOL: worries, } \\
\text { concerns }\end{array}$ & -0.98 & -1.420 & -0.540 & -1.201 & VL & $p<0.001^{a}$ \\
\hline & 36 & Pre-BFB (36) & Post-BFB (36) & $\begin{array}{l}\text { PAC-QOL: satisfac- } \\
\text { tion }\end{array}$ & -1.33 & -1.834 & -0.826 & -1.425 & VL & $p<0.001^{a}$ \\
\hline & 36 & Pre-BFB (36) & Post-BFB (36) & PAC-QOL: overall & -0.92 & -1.277 & -0.563 & -1.393 & VL & $p<0.001^{a}$ \\
\hline \multicolumn{11}{|c|}{ Male chronic pelvic pain syndrome, Urological Chronic Pelvic Pain Syndrome } \\
\hline $\begin{array}{l}\text { Cornel et al. } \\
2005 \text { [34] }\end{array}$ & 31 & $\begin{array}{l}\text { Study group pre-BFB } \\
\text { (31) }\end{array}$ & $\begin{array}{l}\text { Study group } \\
\text { post-BFB (31) }\end{array}$ & $\begin{array}{l}\text { NIH-CPSI: QoL } \\
\text { (0-12 points) }\end{array}$ & -3.80 & n.a. & n.a. & n.a. & n.a. & $p<0.001^{b}$ \\
\hline \multirow[t]{2}{*}{$\begin{array}{l}\text { Yang et al. } \\
2017 \text { [35] }\end{array}$} & 22 & $\begin{array}{l}\text { IG1 (BFB + NMES) } \\
\text { pretreatment (22) }\end{array}$ & $\begin{array}{l}\text { IG1 } 12 \text { weeks } \\
\text { post-treatment } \\
\text { (22) }\end{array}$ & NIH-CPSI: QoL & -5.20 & -7.523 & -2.870 & -1.564 & VL & $p<0.001^{a}$ \\
\hline & 45 & $\mathrm{BFB}+\mathrm{NMES}(22)$ & PEMF (23) & $\begin{array}{l}\text { NIH-CPSI: QoL } 12 \\
\text { wks post }\end{array}$ & 1.20 & -0.382 & 2.782 & 0.365 & S & $p=0.012^{a}$ \\
\hline $\begin{array}{l}\text { He et al. } 2009 \\
{[36]}\end{array}$ & 21 & $\begin{array}{l}\text { Study group pre-BFB } \\
\text { (21) }\end{array}$ & $\begin{array}{l}\text { Study group } \\
10 \text { weeks post- } \\
\text { BFB (21) }\end{array}$ & NIH-CPSI: life impact & -6.70 & -8.605 & -4.795 & -2.528 & $\mathrm{H}$ & $p<0.05^{b}$ \\
\hline \multicolumn{11}{|c|}{ Female chronic pelvic pain } \\
\hline $\begin{array}{l}\text { Gentilcore- } \\
\text { Saulnier et al. } \\
2010 \text { [40] }\end{array}$ & 11 & $\begin{array}{l}\text { IG (provoked vestibu- } \\
\text { lodynia) pre-BFB (11) }\end{array}$ & $\begin{array}{l}\text { IG post-BFB } \\
\text { (11) }\end{array}$ & $\begin{array}{l}\text { Perceived impact } \\
\text { on QoL }(0=\text { no to } \\
10=\text { worst })\end{array}$ & -1.55 & -3.367 & 0.267 & -0.882 & L & $p=0.003^{a}$ \\
\hline $\begin{array}{l}\text { Bendana et al. } \\
2009 \text { [41] }\end{array}$ & 13 & $\begin{array}{l}\text { Strudy group pre-BFB } \\
\text { (52) }\end{array}$ & $\begin{array}{l}\text { Study group } \\
3 \text { months post- } \\
\text { BFB (13) }\end{array}$ & VAS $(0-10)$ & -2.56 & n.a. & n.a. & n.a. & n.a. & $p<0.001^{a}$ \\
\hline
\end{tabular}




\begin{tabular}{|c|c|c|c|c|c|c|c|c|c|c|}
\hline Study & $\begin{array}{l}n \text { (total } \\
\text { group } 1 \text {, } \\
\text { 2) }\end{array}$ & Group 1 (n1) & Group 2 (n2) & Outcome measure & $\begin{array}{l}\text { MD: group } 2 \\
\text { minus } \\
\text { group } 1\end{array}$ & $\begin{array}{l}95 \% \mathrm{Cl} \\
\text { lower } \\
\text { bound }\end{array}$ & $\begin{array}{l}95 \% \mathrm{Cl} \\
\text { upper } \\
\text { bound }\end{array}$ & \multicolumn{2}{|c|}{$\begin{array}{l}\text { Effect size } \\
\text { [strength] }\end{array}$} & $\begin{array}{l}P \text {-value (for } \\
\text { difference in } \\
\text { means) }\end{array}$ \\
\hline \multirow[t]{5}{*}{$\begin{array}{l}\text { Vagedes et al. } \\
2019 \text { [45] }\end{array}$} & 20 & IG1 (BFB) pre-BFB (20) & $\begin{array}{l}\text { IG1 post-BFB } \\
\text { (20) }\end{array}$ & SF-12: mental score & $4.1^{\mathrm{C}}$ & $-0.3^{\mathrm{c}}$ & $8.4^{\mathrm{C}}$ & $0.4^{\mathrm{C}}$ & S & n.a. \\
\hline & 20 & IG1 (BFB) pre-BFB (20) & $\begin{array}{l}\text { IG1 post-BFB } \\
(20)\end{array}$ & $\begin{array}{l}\text { SF-12: physical } \\
\text { score }\end{array}$ & $4.4^{\mathrm{C}}$ & $0.4^{c}$ & $8.5^{c}$ & $0.5^{\mathrm{c}}$ & $S$ & n.a. \\
\hline & 20 & IG1 (BFB) pre-BFB (20) & $\begin{array}{l}\text { IG1 post-BFB } \\
\text { (20) }\end{array}$ & SF-12: sum score & $8.7^{\mathrm{c}}$ & $3.5^{\mathrm{c}}$ & $13.8^{\mathrm{C}}$ & $0.6^{\mathrm{c}}$ & M & n.a. \\
\hline & 37 & $\begin{array}{l}\text { IG1 (BFB) post-BFB } \\
(20)\end{array}$ & $\begin{array}{l}\text { CG (usual care) } \\
\text { (17) }\end{array}$ & SF-12: sum score & $6.13^{\mathrm{C}}$ & $-3.09^{c}$ & $15.35^{c}$ & $0.41^{\mathrm{C}}$ & S & $p>0.05$ \\
\hline & 43 & $\begin{array}{l}\text { IG1 (BFB) post-BFB } \\
(20)\end{array}$ & $\begin{array}{l}\text { IG2 (massage) } \\
\text { post-treatment } \\
\text { (23) }\end{array}$ & SF-12: sum score & $-0.57^{c}$ & $-9.18^{c}$ & $8.03^{c}$ & $-0.04^{c}$ & VS & $p>0.05$ \\
\hline \multicolumn{11}{|c|}{ Chronic pelvic pain in children } \\
\hline $\begin{array}{l}\text { Li et al. } 2006 \\
\text { [54] }\end{array}$ & 22 & $\begin{array}{l}\text { IG patients with } \\
\text { chronic prostatitis } \\
\text { post-BFB (25) }\end{array}$ & IG post-BFB (22) & NIH-CPSI: life impact & 8 & n.a. & n.a. & n.a. & n.a. & $p<0.001^{b}$ \\
\hline \multicolumn{11}{|c|}{ 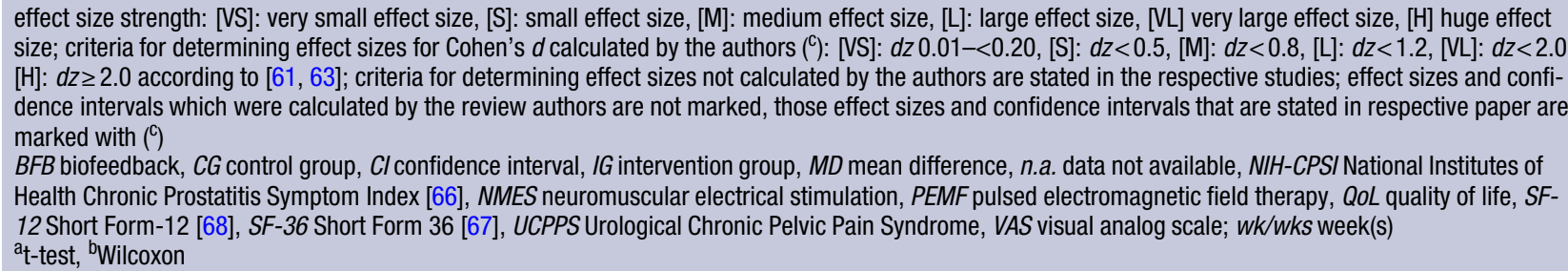 } \\
\hline
\end{tabular}

for multiple testing. Some studies used nonvalidated outcomes to evaluate pain and overall improvement.

The impossibility to fulfil certain quality requirements such as blinding of participants or the administration of placebo treatment, which are standard in pharmacological studies, is immanent to the biofeedback training method and setting.

Given these limitations, the statements that were drawn conducting this review should be understood as tentative evidence and should be considered with caution. Three RCTs of above-average quality with respect to sample size, study design, and reporting [16, $21,45]$ are given special attention in the subsequent discussion.

\section{Efficacy of biofeedback in certain phenotypes and existing recommendations of guidelines}

For anorectal disorders, such as dyssynergic defecation and levator ani syndrome, guidelines exist that state that biofeedback is the preferred treatment for chronic anal pain syndrome (level of evidence IA), [2] and is considered useful in the short-term treatment of levator ani syndrome with dyssynergic defecation (level of evidence IIB) [83]. Biofeedback is recommended for the short-term and long-term treatment of constipation with dyssynergic defecation (level of evidence IA), which is the most common defecation disorder, affecting about $40 \%$ of patients with chronic constipation [83]. Biofeedback seems to benefit patients with dyssynergic defecation above other types of constipation [24, 28, 83, 86]. In PF dyssynergia, a landmark trial by Chiarioni et al. [21] found biofeedback to be superior to laxatives (polyethylene glycol), two other RCTs [87, 88] (not considered in this review) also considered it superior to alternative treatments (diazepam), placebo, sham feedback and standard treatment [86]. The pathophysiology of levator ani syndrome seems to be similar to that of dyssynergic defecation, thus similar techniques and protocols have been used [16]. Both EMG and pressurebased biofeedback therapy protocols appear to be efficacious in restoring a normal pattern of defecation, but larger comparative trials are lacking [83]. Surface EMG probes are cheaper, more durable and usually provide a one or two-channel display [83]. Manometric systems are more expensive, have a multiple channel display and can facilitate rectoanal coordination and sensory training because they have a balloon and rectal sensor $[83,86]$.

In patients with vulvar vestibulitis syndrome (vulvodynia, dyspareunia), preliminary evidence has suggested that altered muscle abnormalities (as shown by altered EMG activity such as elevated resting activity, reduced muscle contraction strength, muscle instability) are present and EMG biofeedback muscle rehabilitation, therefore, is beneficial $[1,38]$. According to Mariani, biofeedback should be used as a firstline treatment in moderate to severe vulvar vestibulitis (together with antidepressants and psychological counseling) [89]. Two uncontrolled studies by Glazer et al. and McKay at al. using portable EMG biofeedback devices showed promising results with this indication [38, 39]. Bergeron et al. (not considered in this 
Table 7 Secondary outcome: Effect of biofeedback interventions on physiological parameters

\begin{tabular}{|c|c|c|c|c|c|c|c|}
\hline Study & $\begin{array}{l}\text { Secondarily } \\
\text { evaluated } \\
\text { outcome } \\
\text { measure }\end{array}$ & Domain, subgroup & $f / u$ & $\begin{array}{l}\text { IG } \\
\text { pre- } \\
\text { post }\end{array}$ & $\begin{array}{l}\text { IG vs. } \\
\text { IG /IG } \\
\text { vs. CG }\end{array}$ & $\begin{array}{l}\text { Significant improvement in sub- } \\
\text { domains, significant difference } \\
\text { between IG/IG or IG/CG (p-value) }\end{array}$ & $\begin{array}{l}\text { No significant improvement in subdo- } \\
\text { mains or no significant difference be- } \\
\text { tween IG/IG, IG/CG ( } p \text {-value) }\end{array}$ \\
\hline \multicolumn{8}{|c|}{ Anorectal pain syndrome } \\
\hline \multirow[t]{4}{*}{$\begin{array}{l}\text { Chiarioni } \\
\text { et al. } 2010 \\
\text { [16] }\end{array}$} & \multirow[t]{4}{*}{$\begin{array}{l}\text { Anorectal } \\
\text { mano- } \\
\text { metry }\end{array}$} & \multirow[t]{2}{*}{$\begin{array}{l}\text { IG1 BFB group: } \\
\text { patients with high } \\
\text { likely LAS }\end{array}$} & $\begin{array}{l}\text { Baseline- } \\
1 \text { month }\end{array}$ & $x$ & - & $\begin{array}{l}\text { Anal pressure with straining }(\% \\
\text { relaxing), balloon defecation }(\% \\
\text { successful), urge threshold }(\mathrm{ml}) \text {, } \\
\text { maximum tolerable volume }(\mathrm{ml}) \text {, } \\
\text { compliance }(\mathrm{mm} \mathrm{Hg})(p<0.025)\end{array}$ & $\begin{array}{l}\text { Resting anal canal pressure }(\mathrm{mm} \mathrm{Hg}) \\
\text { rectoanal inhibitory reflex threshold (ml) } \\
(p \geq 0.025)\end{array}$ \\
\hline & & & $\begin{array}{l}\text { Baseline- } \\
3 \text { month }\end{array}$ & $\mathrm{x}$ & - & $\begin{array}{l}\text { Anal pressure with straining (\% } \\
\text { relaxing), balloon defecation (\% } \\
\text { successful), rectoanal inhibitory } \\
\text { reflex threshold (ml), urge threshold } \\
(\mathrm{ml}), \text { maximum tolerable volume }(\mathrm{ml}) \\
(p<0.025)\end{array}$ & $\begin{array}{l}\text { Resting anal canal pressure }(\mathrm{mm} \mathrm{Hg}) \\
\text { compliance }(\mathrm{mm} \mathrm{Hg})(p \geq 0.025)\end{array}$ \\
\hline & & $\begin{array}{l}\text { IG1 BFB group: pa- } \\
\text { tients with possible } \\
\text { LAS }\end{array}$ & $\begin{array}{l}\text { Baseline-- } \\
1 \text { month, } \\
\text { baseline- } \\
3 \text { months }\end{array}$ & $x$ & - & $\begin{array}{l}\text { Anal pressure with straining (\% } \\
\text { relaxing), balloon defecation (\% } \\
\text { successful) }(p<0.025)\end{array}$ & $\begin{array}{l}\text { Resting anal canal pressure }(\mathrm{mm} \mathrm{Hg}) \text {, } \\
\text { rectoanal inhibitory reflex threshold }(\mathrm{ml}) \text {, } \\
\text { urge threshold }(\mathrm{ml}) \text {, maximum tolera- } \\
\text { ble volume }(\mathrm{ml}) \text {, compliance }(\mathrm{mm} \mathrm{Hg}) \\
(p<0.025)\end{array}$ \\
\hline & & $\begin{array}{l}\text { IG1 BFB group } \\
(n=52) \text { vs. IG2 } \\
\text { EGS }(n=52) \text { or IG3 } \\
\text { massage group } \\
(n=53) \text { (in favour } \\
\text { of BFB) in patients } \\
\text { with high likely LAS }\end{array}$ & $\begin{array}{l}\text { After } \\
1 \text { month, after } \\
3 \text { months }\end{array}$ & - & $\mathrm{x}$ & $\begin{array}{l}\text { Anal pressure with straining ( } \% \\
\text { relaxing), balloon defecation (\% } \\
\text { successful) }(p<0.025)\end{array}$ & $\begin{array}{l}\text { Resting anal canal pressure }(\mathrm{mm} \mathrm{Hg}) \text {, } \\
\text { rectoanal inhibitory reflex threshold }(\mathrm{ml}) \text {, } \\
\text { urge threshold }(\mathrm{ml}) \text {, maximum tolera- } \\
\text { ble volume }(\mathrm{ml}) \text {, compliance }(\mathrm{mm} \mathrm{Hg}) \\
(p<0.025)\end{array}$ \\
\hline $\begin{array}{l}\text { Heah et al. } \\
1997[17]\end{array}$ & $\begin{array}{l}\text { Anorectal } \\
\text { mano- } \\
\text { metry }\end{array}$ & Study group $(n=16)$ & $\begin{array}{l}\text { Baseline- } \\
\text { after treat- } \\
\text { ment }\end{array}$ & $\mathrm{x}$ & - & None $\left(p<0.05^{2}\right)$ & $\begin{array}{l}\text { Anal canal mean resting/maximum } \\
\text { squeeze pressure }(\mathrm{mm} \mathrm{Hg}) \text {, rectum } \\
\text { volume first sensation }(\mathrm{ml}) / \text { maximum } \\
\text { tolerable volume }(\mathrm{ml}) / \text { compliance } \\
(\mathrm{ml} / \mathrm{mm} \mathrm{Hg}) \text {, perineal descent rest/strain } \\
(\mathrm{cm})(p>0.05)\end{array}$ \\
\hline $\begin{array}{l}\text { Grimaud } \\
\text { et al. } 1991 \\
\text { [20] }\end{array}$ & $\begin{array}{l}\text { Anorectal } \\
\text { mano- } \\
\text { metry }\end{array}$ & Study group $(n=12)$ & $\begin{array}{l}\text { Baseline-- } \\
\text { after treat- } \\
\text { ment }\end{array}$ & $x$ & - & $\begin{array}{l}\text { Anal canal resting pressure ( } \mathrm{mm} \mathrm{Hg} \text { ), } \\
p<0.01 \text { (no significant difference } \\
\text { any more compared to healthy } \\
\text { controls without BFB intervention) }\end{array}$ & - \\
\hline \multicolumn{8}{|c|}{ Constipation, dyssynergic defecation } \\
\hline \multirow[t]{4}{*}{$\begin{array}{l}\text { Chiarioni } \\
\text { et al. } 2006 \\
\text { [21] }\end{array}$} & \multirow[t]{4}{*}{$\begin{array}{l}\text { Anorectal } \\
\text { mano- } \\
\text { metry }\end{array}$} & \multirow[t]{2}{*}{$\begin{array}{l}\text { BFB group } \\
(n=54 / 109)\end{array}$} & $\begin{array}{l}\text { Baseline-- } \\
6 \text { months, } \\
\text { baseline-- } \\
12 \text { months } \\
\text { after starting } \\
\text { treatment }\end{array}$ & $x$ & - & $\begin{array}{l}\text { Increased anal pressure }(n, \%), \\
\text { (paradoxical) EMG increase }(n, \%) \text {, } \\
\text { unable to evacuate balloon }(n, \%) \text {, } \\
\text { anal squeeze pressure (mm Hg), } \\
\text { rectoanal inhibitory reflex threshold } \\
(\mathrm{ml}) \text {, urge threshold }(\mathrm{ml}) \text {, maximum } \\
\text { tolerable volume }(\mathrm{ml}) \text {, compliance } \\
(\mathrm{mm} \mathrm{Hg})(p<0.01)\end{array}$ & Anal resting pressure $(\mathrm{mm} \mathrm{Hg})(p \geq 0.01)$ \\
\hline & & & $\begin{array}{l}\text { Baseline- } \\
24 \text { months } \\
\text { after starting } \\
\text { treatment }\end{array}$ & $x$ & - & $\begin{array}{l}\text { Increased anal pressure }(n, \%) \text {, } \\
\text { (paradoxical) EMG increase }(n, \%) \text {, } \\
\text { unable to evacuate balloon }(n, \%), \\
\text { anal squeeze pressure }(\mathrm{mm} \mathrm{Hg}), \\
\text { urge threshold }(\mathrm{ml}), \text { maximum toler- } \\
\text { able volume }(\mathrm{ml})(p<0.01)\end{array}$ & $\begin{array}{l}\text { Anal resting pressure }(\mathrm{mm} \mathrm{Hg}) \text {, rectoanal } \\
\text { inhibitory reflex threshold }(\mathrm{ml}) \text {, compli- } \\
\text { ance }(\mathrm{mm} \mathrm{Hg})(p \geq 0.01)\end{array}$ \\
\hline & & \multirow[t]{2}{*}{$\begin{array}{l}\text { BFB group vs. laxa- } \\
\text { tive group, in favour } \\
\text { BFB group ( } n=109)\end{array}$} & $\begin{array}{l}6 \text { and after } \\
12 \text { months } \\
\text { after starting } \\
\text { treatment }\end{array}$ & - & $x$ & $\begin{array}{l}\text { Increased anal pressure }(n, \%), \\
\text { (paradoxical) EMG increase }(n, \%) \text {, } \\
\text { unable to evacuate balloon }(n, \%) \\
(p<0.01)\end{array}$ & $\begin{array}{l}\text { Anal resting pressure }(\mathrm{mm} \mathrm{Hg}) \text {, anal } \\
\text { squeeze pressure }(\mathrm{mm} \mathrm{Hg}) \text {, rectoanal in- } \\
\text { hibitory reflex threshold }(\mathrm{ml}) \text {, urge thresh- } \\
\text { old }(\mathrm{ml}) \text {, maximum tolerable volume }(\mathrm{ml}) \text {, } \\
\text { compliance }(\mathrm{mm} \mathrm{Hg})\end{array}$ \\
\hline & & & $\begin{array}{l}24 \text { months } \\
\text { after starting } \\
\text { treatment }\end{array}$ & - & $\mathrm{x}$ & No parameter $(p<0.01)$ & All parameters $(p \geq 0.01)$ \\
\hline
\end{tabular}


Table 7 (Continued)

\begin{tabular}{|c|c|c|c|c|c|c|c|}
\hline Study & $\begin{array}{l}\text { Secondarily } \\
\text { evaluated } \\
\text { outcome } \\
\text { measure }\end{array}$ & Domain, subgroup & $f / u$ & $\begin{array}{l}\text { IG } \\
\text { pre- } \\
\text { post }\end{array}$ & $\begin{array}{l}\text { IG vs. } \\
\text { IG /IG } \\
\text { vs. CG }\end{array}$ & $\begin{array}{l}\text { Significant improvement in sub- } \\
\text { domains, significant difference } \\
\text { between IG/IG or IG/CG ( } p \text {-value) }\end{array}$ & $\begin{array}{l}\text { No significant improvement in subdo- } \\
\text { mains or no significant difference be- } \\
\text { tween IG/IG, IG/CG (p-value) }\end{array}$ \\
\hline $\begin{array}{l}\text { Koutsoma- } \\
\text { nis et al. } \\
1994 \text { [22] }\end{array}$ & $\begin{array}{l}\text { Anorectal } \\
\text { mano- } \\
\text { metry }\end{array}$ & Study group $(n=20)$ & $\begin{array}{l}\text { Baseline-- } \\
\text { after treat- } \\
\text { ment }\end{array}$ & $x$ & - & $\begin{array}{l}\text { Paradoxical contraction on evacu- } \\
\text { ation straining ( } n \text { pre: } n=15 / 20, n \\
\text { post: } n=0 / 20,(p: \text { n.a.)) }\end{array}$ & $\begin{array}{l}\text { Anal resting pressure, anal squeeze } \\
\text { pressure, anorectal sensation ( } p: \text { n.a.) }\end{array}$ \\
\hline \multirow[t]{2}{*}{$\begin{array}{l}\text { Battaglia } \\
\text { et al. } 2004 \\
\text { [24] }\end{array}$} & \multirow[t]{2}{*}{$\begin{array}{l}\text { Anorectal } \\
\text { mano- } \\
\text { metry }\end{array}$} & $\begin{array}{l}\text { Patients with } \\
\text { PF dyssynergia } \\
\text { ( } n=14 / 24)\end{array}$ & $\begin{array}{l}\text { Baseline- } \\
3 \text { months af- } \\
\text { ter treatment }\end{array}$ & $x$ & - & $\begin{array}{l}\text { Sensation threshold ( } \mathrm{mm} \mathrm{Hg} ; \\
p=0.042 \text { ), paradoxical increase } \\
\text { in intra-anal pressure during strain- } \\
\text { ing ( } p \text { : n.a.) }\end{array}$ & $\begin{array}{l}\text { Maximum basal pressure of internal anal } \\
\text { sphincter, maximum rectum tolerable } \\
\text { volume }(p \geq 0.05)\end{array}$ \\
\hline & & $\begin{array}{l}\text { Patients with slow } \\
\text { transit }(n=10 / 24)\end{array}$ & $\begin{array}{l}\text { Baseline-- } \\
3 \text { months af- } \\
\text { ter treatment }\end{array}$ & $x$ & - & $\begin{array}{l}\text { Maximum rectum tolerable volume } \\
(\mathrm{ml}),(p=0.008)\end{array}$ & $\begin{array}{l}\text { Maximum basal pressure of internal anal } \\
\text { sphincter (mm Hg), sensation threshold } \\
(\mathrm{mm} \mathrm{Hg})(p \geq 0.05)\end{array}$ \\
\hline $\begin{array}{l}\text { Wang et al. } \\
2003 \text { [25] }\end{array}$ & $\begin{array}{l}\text { Anorectal } \\
\text { mano- } \\
\text { metry }\end{array}$ & Study group $(n=50)$ & $\begin{array}{l}\text { Baseline- } \\
\text { after treat- } \\
\text { ment }\end{array}$ & $x$ & - & $\begin{array}{l}\text { Anal canal average rest pressure } \\
(\mathrm{mm} \mathrm{Hg}) \text { rectum: initial sense }(\mathrm{ml}) \text {, } \\
(p<0.05)\end{array}$ & $\begin{array}{l}\text { Anal canal voluntary squeeze }(\mathrm{mm} \mathrm{Hg}) \text {, } \\
\text { rectum: maximum tolerable volume }(\mathrm{ml}) \\
\text { and compliance }(\mathrm{ml} / \mathrm{mm} \mathrm{Hg}),(p \geq 0.05)\end{array}$ \\
\hline $\begin{array}{l}\text { Ba-Bai-Ke- } \\
\text { Re et al. } \\
2014 \text { [26] }\end{array}$ & $\begin{array}{l}\text { Anorectal } \\
\text { mano- } \\
\text { metry }\end{array}$ & $\begin{array}{l}\text { BFB group vs. laxa- } \\
\text { tive group, in favor } \\
\text { BFB group }(n=88)\end{array}$ & $\begin{array}{l}\text { Baseline-- } \\
\text { after treat- } \\
\text { ment }\end{array}$ & - & $x$ & $\begin{array}{l}\text { Anorectal resting pressure, anorec- } \\
\text { tal squeeze pressure }(\mathrm{mm} \mathrm{Hg}) \\
(p<0.05)\end{array}$ & - \\
\hline \multirow[t]{5}{*}{$\begin{array}{l}\text { Chiarioni } \\
\text { et al. } 2005 \\
\text { [28] }\end{array}$} & $\begin{array}{l}\text { Gut transit } \\
\text { time }\end{array}$ & $\begin{array}{l}\text { PF dyssynergia } \\
(n=34) \text { vs. slow } \\
\text { transit only }(n=12)\end{array}$ & $\begin{array}{l}\text { Baseline-- } \\
1 / 6 / 12 / 24 \\
\text { months after } \\
\text { treatment }\end{array}$ & - & $x$ & $\begin{array}{l}\% \text { of patients with abnormal transit } \\
\text { test: baseline: } 100 \% \text {; at all f/u: PF } \\
\text { dyssynergia vs. slow-transit-only: } \\
\text { sign. smaller } \% \text { of patients with } \\
\text { abnormally delayed transit, } p<0.05\end{array}$ & - \\
\hline & $\begin{array}{l}\text { Balloon } \\
\text { defecation } \\
\text { test }\end{array}$ & $\begin{array}{l}\text { Patients with PF } \\
\text { dyssynergia }(n=34)\end{array}$ & $\begin{array}{l}\text { Baseline-- } \\
\text { after treat- } \\
\text { ment }(1, \\
6,12 \text {, } \\
24 \text { months) }\end{array}$ & $\mathrm{x}$ & - & $\begin{array}{l}\text { Baseline: } 0 \% \text {, after treatment } \\
\text { (1-24 months): } 82-85 \% \text { could } \\
\text { defecate the balloon within } 5 \text { min } \\
\text { (p: n.a.) }\end{array}$ & - \\
\hline & \multirow[t]{3}{*}{$\begin{array}{l}\text { Anorectal } \\
\text { mano- } \\
\text { metry }\end{array}$} & $\begin{array}{l}\text { Patients with PF } \\
\text { dyssynergia ( } n=34)\end{array}$ & $\begin{array}{l}\text { Baseline- } \\
\text { after treat- } \\
\text { ment (1, } \\
6 \text { months) }\end{array}$ & $x$ & - & $\begin{array}{l}\text { Urge threshold }(\mathrm{ml}) \text {, maximum tol- } \\
\text { erable pressure }(\mathrm{mm} \mathrm{Hg}) \text {, straining } \\
\text { rectal pressure }(\mathrm{mm} \mathrm{Hg}) \text {, dyssyner- } \\
\text { gia (balloon defecation test) } \\
(p<0.05)\end{array}$ & $\begin{array}{l}\text { Anal canal resting pressure }(\mathrm{ml}) \text {, rec- } \\
\text { toanal inhibitory reflex threshold (ml), } \\
\text { compliance (mm Hg100ml) } p \geq 0.05\end{array}$ \\
\hline & & $\begin{array}{l}\text { Patients with slow } \\
\text { transit only }(n=12)\end{array}$ & $\begin{array}{l}\text { Baseline- } \\
\text { after treat- } \\
\text { ment (1, } \\
6 \text { months) }\end{array}$ & $x$ & - & Urge threshold $(\mathrm{ml})(p<0.05)$ & $\begin{array}{l}\text { Anal canal resting pressure }(\mathrm{ml}) \text {, rec- } \\
\text { toanal inhibitory reflex threshold }(\mathrm{ml}) \text {, } \\
\text { maximum tolerable pressure }(\mathrm{mm} \mathrm{Hg}) \text {, } \\
\text { compliance }(\mathrm{mm} \mathrm{Hg} 100 \mathrm{ml}) \text {, straining } \\
\text { rectal pressure }(\mathrm{mm} \mathrm{Hg})(p \geq 0.05)\end{array}$ \\
\hline & & $\begin{array}{l}\text { Patients with PF } \\
\text { dyssynergia }(n=34) \\
\text { vs. slow transit only } \\
(n=12) \text { in favour of } \\
\text { PF dyssynergia }\end{array}$ & $\begin{array}{l}\text { After } 1 \text {, } \\
6 \text { months }\end{array}$ & - & $x$ & $\begin{array}{l}\text { Rectoanal inhibitory reflex threshold } \\
\text { (ml; only after } 1 \text { month), after } 1 \text {, } \\
6 \text { months: urge threshold (ml), max- } \\
\text { imum tolerable pressure (mm Hg), } \\
p<0.01\end{array}$ & $\begin{array}{l}\text { Rectoanal inhibitory reflex thresh- } \\
\text { old (ml, only after } 6 \text { months), after } 1 \text {, } \\
6 \text { months: anal canal resting pressure } \\
\text { (ml), rectoanal inhibitory reflex threshold } \\
\text { (ml, only after } 6 \text { months), compliance } \\
(\mathrm{mm} \mathrm{Hg} 100 \mathrm{ml}) \text {, straining rectal pressure } \\
(\mathrm{mm} \mathrm{Hg})(p \geq 0.01)\end{array}$ \\
\hline \multirow[t]{4}{*}{$\begin{array}{l}\text { Parker et } \\
\text { al. } 2019 \\
\text { [31] }\end{array}$} & \multirow[t]{4}{*}{$\begin{array}{l}\text { Anorectal } \\
\text { mano- } \\
\text { metry }\end{array}$} & $\begin{array}{l}\text { Whole study group } \\
\text { (constipation, fecal } \\
\text { incontinence, rectal } \\
\text { pain) }(n=130)\end{array}$ & $\begin{array}{l}\text { Baseline-- } \\
\text { after treat- } \\
\text { ment }\end{array}$ & $\mathrm{x}$ & - & $\begin{array}{l}\text { Resolved dyssynergic manometric } \\
\text { pressure profile, balloon expulsion } \\
\text { test }<1 \text { min in } n=27 / 130 \text {, (p: n.a.) }\end{array}$ & - \\
\hline & & $\begin{array}{l}\text { Constipation + dyssyI } \\
\text { defecation } \\
(n=33 / 130)\end{array}$ & $\begin{array}{l}\text { Baseline-- } \\
\text { after treat- } \\
\text { ment }\end{array}$ & $x$ & - & $\begin{array}{l}\text { Resolved dyssynergic manometric } \\
\text { pressure profile, balloon expulsion } \\
\text { test }<1 \text { min in } n=13 / 53 \text {, ( } p: \text { n.a.) }\end{array}$ & - \\
\hline & & $\begin{array}{l}\text { Constipation without } \\
\text { dyssynergic defe- } \\
\text { cation ( } n=3 / 130)\end{array}$ & $\begin{array}{l}\text { Baseline- } \\
\text { after treat- } \\
\text { ment }\end{array}$ & $x$ & - & $\begin{array}{l}\text { Improvement in anorectal manom- } \\
\text { etry profile (resolved dyssynergic } \\
\text { manometric pressure profile, balloon } \\
\text { expulsion test }<1 \mathrm{~min} \text { ) in } n=2 / 3, \\
\text { (p: n.a.) }\end{array}$ & - \\
\hline & & $\begin{array}{l}\text { Rectal pain } \\
(n=3 / 130)\end{array}$ & $\begin{array}{l}\text { Baseline-- } \\
\text { after treat- } \\
\text { ment }\end{array}$ & $x$ & - & $\begin{array}{l}\text { Resolved dyssynergic manometric } \\
\text { pressure profile, balioon expulsion } \\
\text { test }<1 \text { min in } n=2 / 3 \text {, (p: n.a.) }\end{array}$ & - \\
\hline
\end{tabular}


Table 7 (Continued)

\begin{tabular}{|c|c|c|c|c|c|c|c|}
\hline Study & $\begin{array}{l}\text { Secondarily } \\
\text { evaluated } \\
\text { outcome }\end{array}$ & Domain, subgroup & $f / u$ & $\begin{array}{l}\text { IG } \\
\text { pre- } \\
\text { post }\end{array}$ & $\begin{array}{l}\text { IG vs. } \\
\text { IG /IG } \\
\text { vs. CG }\end{array}$ & $\begin{array}{l}\text { Significant improvement in sub- } \\
\text { domains, significant difference } \\
\text { between IG/IG or IG/CG (p-value) }\end{array}$ & $\begin{array}{l}\text { No significant improvement in subdo- } \\
\text { mains or no significant difference be- } \\
\text { tween IG/IG, IG/CG (p-value) }\end{array}$ \\
\hline
\end{tabular}

\section{Male chronic pelvic pain syndrome, Urological Chronic Pelvic Pain Syndrome}

\begin{tabular}{|c|c|c|c|c|c|c|}
\hline $\begin{array}{l}\text { Cornel et } \\
\text { al. } 2005 \\
{[34]}\end{array}$ & $\begin{array}{l}\text { Levator ani } \\
\text { EMG }\end{array}$ & Study group $(n=18)$ & $\begin{array}{l}\text { Baseline- } \\
\text { after treat- } \\
\text { ment }\end{array}$ & $x$ & - & $\begin{array}{l}\text { Mean pelvic muscle tonus } \downarrow \text { (mcV, } \\
p<0.001^{2} \text { ) }\end{array}$ \\
\hline $\begin{array}{l}\text { He et al. } \\
2010[36]\end{array}$ & $\begin{array}{l}\text { Urodyna- } \\
\text { mics } \\
\text { (uroflowme- } \\
\text { try+ EMG) }\end{array}$ & Study group $(n=21)$ & $\begin{array}{l}\text { Baseline- } \\
10 \text { wks after } \\
\text { treatment }\end{array}$ & $x$ & - & $\begin{array}{l}\text { Max. flow rate }(\mathrm{ml} / \mathrm{s}) \text {, max. detrusor } \\
\text { pressure-storage phase }(\mathrm{cmH} 20) \text {, } \\
\text { max. urethra closure pressure } \\
(\mathrm{cmH} 20) \text {, max. urethral pressure } \\
(\mathrm{cmH} 20)(p<0.05)\end{array}$ \\
\hline
\end{tabular}

\section{Female chronic pelvic pain}

\begin{tabular}{|c|c|c|c|c|c|c|c|}
\hline $\begin{array}{l}\text { Glazer et } \\
\text { al. } 1995 \\
\text { [38] }\end{array}$ & PF EMG & Study group $(n=33)$ & $\begin{array}{l}\text { Baseline-- } \\
\text { after treat- } \\
\text { ment (=after } \\
6 \text { th assess- } \\
\text { ment) }\end{array}$ & $x$ & - & $\begin{array}{l}\text { Muscle contractile strength }=\text { mean } \\
\text { contraction amplitude (mcV) } \uparrow \text {, } \\
\text { mean relaxation amplitude } \downarrow(\mathrm{mcV}) \\
(p<0.0001) \text {, SD: measure of the } \\
\text { stability of the muscle at rest im- } \\
\text { proved ( } p \text { : } \mathrm{n} . \mathrm{a} .)\end{array}$ & - \\
\hline $\begin{array}{l}\text { McKay et } \\
\text { al. } 2001 \\
\text { [39] }\end{array}$ & PF EMG & Study group $(n=29)$ & $\begin{array}{l}\text { After } \\
1 \text { month- } \\
\text { after } \\
6 \text { months }\end{array}$ & $x$ & - & $\begin{array}{l}\text { Mean maximum contractile strength } \\
\uparrow(\mathrm{mcV}) \text { : after } 1^{\text {st }} \text { month: } 16.42 \text { in } \\
n=29 \text {, after } 6^{\text {th }} \text { month: } 42.73 \text { in } \\
n=11 \text { (dropout } n=18),(p: \text { n.a.) }\end{array}$ & - \\
\hline \multirow{8}{*}{$\begin{array}{l}\text { Gentilcore- } \\
\text { Saulnier et } \\
\text { al. } 2010 \\
{[40]}\end{array}$} & \multirow[t]{5}{*}{ PF EMG } & \multirow[t]{3}{*}{$\begin{array}{l}\text { IG (provoked } \\
\text { vestibulodynia, } \\
n=11 \text { ) }\end{array}$} & $\begin{array}{l}\text { Baseline- } \\
\text { after treat- } \\
\text { ment }\end{array}$ & $x$ & - & - & $\begin{array}{l}\text { Tonic surface EMG resting activity: deep } \\
(p=0.86) \text { or superficial }(p=0.32) \text { PF } \\
\text { muscle layer }\end{array}$ \\
\hline & & & $\begin{array}{l}\text { Baseline- } \\
\text { after treat- } \\
\text { ment }\end{array}$ & $\mathrm{x}$ & - & - & $\begin{array}{l}\text { PF muscle maximum voluntary contrac- } \\
\text { tile activity: deep: } p=0.82 \text {; superficial: } \\
p=0.50\end{array}$ \\
\hline & & & $\begin{array}{l}\text { Baseline- } \\
\text { after treat- } \\
\text { ment }\end{array}$ & $x$ & - & $\begin{array}{l}\text { Superficial PFM EMG activity pain } \\
\text { responses } \downarrow(\mathrm{mcV}),(p<0.0001)\end{array}$ & $\begin{array}{l}\text { Deep PF layer EMG activity pain response } \\
(\mathrm{mcV}),(p=0.72)\end{array}$ \\
\hline & & $\begin{array}{l}\text { IG (provoked } \\
\text { vestibulodynia, } \\
n=11) \text { vs. healthy } \\
\text { CG }(n=11)\end{array}$ & $\begin{array}{l}\text { Baseline, } \\
\text { pretreatment }\end{array}$ & - & $x$ & $\begin{array}{l}\text { Sign. greater superficial PFM EMG } \\
\text { activity pain responses (mcV) in } \\
\text { pretreatment IG compared to CG } \\
\text { ( } p=0.003) \text {; sign. higher tonic activ- } \\
\text { ity in superficial PFM in pretreatment } \\
\text { IG compared to CG }(p=0.04)\end{array}$ & $\begin{array}{l}\text { No sign. difference pretreatment IG } \\
\text { vs. CG for: PFM maximum voluntary } \\
\text { contractile activity for deep }(p=0.81) \\
\text { and superficial }(p=0.36) \text { PFM; EMG pain } \\
\text { responses of the deep PFM }(p=0.89) \text {; } \\
\text { deep PFM tonic activity }(p=0.18)\end{array}$ \\
\hline & & $\begin{array}{l}\text { IG (provoked } \\
\text { vestibulodynia, } \\
n=11) \text { vs. healthy } \\
\text { CG }(n=11)\end{array}$ & $\begin{array}{l}\text { After treat- } \\
\text { ment }\end{array}$ & - & $x$ & - & $\begin{array}{l}\text { No sign. difference between posttreat- } \\
\text { ment IG and CG: tonic surface EMG rest- } \\
\text { ing activity at both superficial }(p=0.82) \\
\text { and deep ( } p=0.31) \text { PFM; PFM maximum } \\
\text { voluntary contractile activity for deep } \\
(p=0.54) \text { and superficial }(p=0.90) \text { PFM; } \\
\text { EMG activity pain response (mcV) for } \\
\text { deep ( } p=0.98) \text { or superficial }(p=0.18) \\
\text { PFM }\end{array}$ \\
\hline & \multirow[t]{3}{*}{$\begin{array}{l}\text { Digital } \\
\text { intravaginal } \\
\text { assessment }\end{array}$} & $\begin{array}{l}\text { IG ( } n=11 \text { with } \\
\text { pelvic floor dyssyn- } \\
\text { ergia) }\end{array}$ & $\begin{array}{l}\text { Baseline-- } \\
\text { after treat- } \\
\text { ment }\end{array}$ & $x$ & - & $\begin{array}{l}\text { PFM tone } \downarrow(p<0.001) \text {, PFM flexibil- } \\
\text { ity } \uparrow(p=0.01) \text {, PFM post-contraction } \\
\text { relaxation capacity } \uparrow(p=0.05) \text {, PFM } \\
\text { strength } \uparrow(p=0.04)\end{array}$ & - \\
\hline & & $\begin{array}{l}\text { IG }(n=11 \text { with } \\
\text { provoked vestibulo- } \\
\text { dynia) vs. healthy } \\
\text { CG }(n=11)\end{array}$ & Baseline & - & $x$ & $\begin{array}{l}\text { Pretreatment PVD group vs. CG: } \\
\text { PFM tone: sign. higher in PVD group } \\
\text { ( } p=0.005), \text { PFM flexibility: sign. } \\
\text { lower in PVD group ( } p=0.01), \text { PFM } \\
\text { relaxation: sign. less ability to re- } \\
\text { lax PFM in PVD group ( } p=0.02) \\
\text { compared to CG }\end{array}$ & $\begin{array}{l}\text { Pretreatment PVD group vs. CG: no } \\
\text { significant difference in PFM strength } \\
(p=0.54)\end{array}$ \\
\hline & & $\begin{array}{l}\text { IG }(n=11 \text { patients } \\
\text { with provoked } \\
\text { vestibulodynia) vs. } \\
\text { healthy CG }(n=11)\end{array}$ & $\begin{array}{l}\text { After treat- } \\
\text { ment }\end{array}$ & - & $x$ & - & $\begin{array}{l}\text { posttreatment PVD group vs. CG: no } \\
\text { sign. difference in PFM tone }(p=0.30) \text {, } \\
\text { PFM flexibility }(p=1.00) \text {, PFM relaxation } \\
(p=0.47) \text {, PFM strength }(p=0.12)\end{array}$ \\
\hline $\begin{array}{l}\text { Philips et } \\
\text { al. } 1992 \\
\text { [42] }\end{array}$ & $\begin{array}{l}\text { Perivaginal } \\
\text { EMG }\end{array}$ & $\begin{array}{l}\text { IG } 1 \text { (BFB group, } \\
n=10)\end{array}$ & $\begin{array}{l}\text { Baseline-- } \\
\text { after treat- } \\
\text { ment }\end{array}$ & $x$ & - & - & $\begin{array}{l}\text { Mean EMG scores (seated to void, tens- } \\
\text { ing, relaxing, voiding; mcV) } p>0.05\end{array}$ \\
\hline
\end{tabular}




\begin{tabular}{|c|c|c|c|c|c|c|c|}
\hline Study & $\begin{array}{l}\text { Secondarily } \\
\text { evaluated } \\
\text { outcome } \\
\text { measure }\end{array}$ & Domain, subgroup & $f / u$ & $\begin{array}{l}\text { IG } \\
\text { pre- } \\
\text { post }\end{array}$ & $\begin{array}{l}\text { IG vs. } \\
\text { IG /IG } \\
\text { vs. CG }\end{array}$ & $\begin{array}{l}\text { Significant improvement in sub- } \\
\text { domains, significant difference } \\
\text { between IG/IG or IG/CG (p-value) }\end{array}$ & $\begin{array}{l}\text { No significant improvement in subdo- } \\
\text { mains or no significant difference be- } \\
\text { tween IG/IG, IG/CG (p-value) }\end{array}$ \\
\hline $\begin{array}{l}\text { Bennink et } \\
\text { al. } 1982 \\
\text { [44] }\end{array}$ & $\begin{array}{l}\text { EMG lower } \\
\text { abdomen }\end{array}$ & $\begin{array}{l}\text { IG1 }(n=5) \text { vs. IG2 } \\
(n=5)\end{array}$ & - & - & $x$ & $\begin{array}{l}\text { BFB group maintained a significantly } \\
\text { lower level of EMG muscle tone } \\
\left(\mathrm{mcV} / \mathrm{s} \text { ) of lower abdomen on } 1^{\text {st }}\right. \\
\text { day of menstruation compared to } \\
\text { massage group }(p<0.05)\end{array}$ & - \\
\hline \multirow[t]{2}{*}{$\begin{array}{l}\text { Vagedes et } \\
\text { al. } 2019 \\
{[45]}\end{array}$} & \multirow[t]{2}{*}{$\begin{array}{l}\text { Heart rate } \\
\text { variability }\end{array}$} & BFB group $(n=20)$ & $\begin{array}{l}\text { Baseline-- } \\
\text { after treat- } \\
\text { ment }\end{array}$ & $x$ & - & - & SDNNI, RMSSD, LF/HF ratio $(p>0.05)$ \\
\hline & & $\begin{array}{l}\text { BFB }(n=20) \text { vs. CG } \\
(n=17) / \mathrm{BFB} \text { vs. } \\
\text { massage }(n=23)\end{array}$ & $\begin{array}{l}\text { After treat- } \\
\text { ment }\end{array}$ & - & $x$ & - & $\begin{array}{l}\text { Same values: BFB vs. CG/massage vs. } \\
\text { BFB group post treatment: } p>0.05\end{array}$ \\
\hline $\begin{array}{l}\text { Lúcio et al. } \\
2014 \text { [47] }\end{array}$ & $\begin{array}{l}\text { Intravaginal } \\
\text { digital ex- } \\
\text { amination }\end{array}$ & $\begin{array}{l}\text { IG1: EMG BFB + PF } \\
\text { training + sham } \\
\text { NMES }(n=6)\end{array}$ & $\begin{array}{l}\text { Baseline- } \\
\text { after treat- } \\
\text { ment }\end{array}$ & $x$ & - & $\begin{array}{l}\text { PF muscle function according to } \\
\text { PERFECT scheme } \uparrow[69] \text { : power } \\
\text { (0-5= max. strength), endurance } \\
\text { (sec), dynamic endurance (no. of } \\
\text { repetitions), fast contractions (no. of } \\
\text { repetitions): } p<0.05\end{array}$ & $\begin{array}{l}\text { PF muscle palpation score: PF muscle } \\
\text { tone (score: }-3 \text { to }+3=\text { very hyper- } \\
\text { tonic), flexibility (score } 0-4=\text { very flex- } \\
\text { ible), ability to relax PF muscles (Score } \\
0-4=\text { spastic): } p>0.05\end{array}$ \\
\hline \multicolumn{8}{|c|}{ Chronic pelvic pain in children } \\
\hline \multirow[t]{2}{*}{$\begin{array}{l}\text { Ebiloglu et } \\
\text { al. 2016 } \\
\text { [52] }\end{array}$} & Urodynamics & $\begin{array}{l}\text { Study group } \\
(n=136)\end{array}$ & $\begin{array}{l}\text { Baseline- } \\
\text { after treat- } \\
\text { ment } \\
\text { ( } 6 \text { months })\end{array}$ & $\mathrm{x}$ & - & $\begin{array}{l}\text { No. of patients with positive per- } \\
\text { ineal EMG activity while urinating } \\
(p<0.001)\end{array}$ & - \\
\hline & Urodynamics & $\begin{array}{l}\text { Study group } \\
(n=136)\end{array}$ & $\begin{array}{l}\text { Baseline- } \\
\text { after treat- } \\
\text { ment } \\
\text { ( } 6 \text { months) }\end{array}$ & $\mathrm{x}$ & - & $\begin{array}{l}\text { Mean voided volume }(\mathrm{ml}, p=0.019) \text {, } \\
\text { mean maximum flow rate }(\mathrm{ml} / \mathrm{s}, \\
p=0.012)\end{array}$ & $\begin{array}{l}\text { Mean average flow rate }(\mathrm{ml} / \mathrm{s}, p=0.209) \text {, } \\
\text { mean voiding time }(\mathrm{s}, p=0.345) \text {, post- } \\
\text { void residual volume (ml, } p=0.374)\end{array}$ \\
\hline $\begin{array}{l}\text { Ergin et al. } \\
2016[53]\end{array}$ & Urodynamics & $\begin{array}{l}\text { Intervention group } \\
(n=39)\end{array}$ & $\begin{array}{l}\text { Baseline- } \\
\text { after treat- } \\
\text { ment } \\
\text { ( } 6 \text { months })\end{array}$ & $x$ & - & $\begin{array}{l}\text { Uroflowmetry-EMG, post-void } \\
\text { residual volume }(p<0.001)\end{array}$ & - \\
\hline $\begin{array}{l}\text { Li et al. } \\
2006[54]\end{array}$ & Urodynamics & $\mathrm{IG}(n=25)$ & $\begin{array}{l}\text { Baseline- } \\
\text { after treat- } \\
\text { ment }\end{array}$ & $\mathrm{x}$ & - & $\begin{array}{l}\text { Maximum urinary flow rate }(\mathrm{ml} / \mathrm{s}) \text {, } \\
p=0.001\end{array}$ & $\begin{array}{l}\text { Postvoid residual urine volume (ml), } \\
p=0.08\end{array}$ \\
\hline
\end{tabular}

$B F B$ biofeedback, $C G$ control group (no intervention), EGS electrogalvanic stimulation, EMG electromyography, f/u follow-up, IG intervention group, LAS levator ani syndrome, LF/HF ratio ratio of two bands from frequency domain analysis: $\mathrm{LF}$ band $(0.04-0.15 \mathrm{~Hz})$ indicating sympathetic and parasympathetic activity, $\mathrm{HF}$ band $(0.15-0.40 \mathrm{~Hz})$ indicating parasympathetic activity; $\mathrm{mcV}$ microvolt, $\mathrm{m} / \mathrm{mililiter}, \mathrm{ml} /$ mililiter per second, no. number(s), $P F$ pelvic floor, $P F M$ pelvic floor muscle(s), RMSSD root mean square of successive differences; $s$ second(s), $S D$ standard deviation, $S D N N$ standard deviation of normal to normal, vs. versus, $w k$, wks week(s)

review) applied the home-based Glazer protocol in an RCT design. They confirmed that EMG biofeedback as well as cognitive-behavioral therapy and vestibulectomy, could improve sexual function and reduce pain (greatest pain reduction in the vestibulectomy group $[1,90,91])$ in the short and long term.

\section{Pros and cons of biofeedback in pelvic pain conditions and criteria to improve treatment success}

Biofeedback is a safe method, which has not shown any significant adverse effects. This might make biofeedback an attractive treatment option even in indications with a smaller success rate. As biofeedback is a labor-intensive approach [83] and quite time-consuming for both therapist and patient, it is important to preselect those patients who have a high chance of benefitting from the intervention.

The use of biofeedback to treat pelvic pain is based on the idea that these pain conditions may result from, or are associated with, pelvic floor muscle dysfunction. Digital palpation of pelvic floor muscles should be integrated into routine examination to identify myofascial pain as a primary or contributing source of pelvic pain condition [2, 8]. In anorectal pain conditions, tenderness on rectal examination has shown to be a valid criterion of treatment success [16]. Shoskes et al. identified and grouped six clinical phenotypes (urinary, psychosocial, organ-specific, infection, neurologic, tenderness of skeletal muscles) in the UPOINT classification in patients with urologic CPPS [2, 92, 93]. This classification was implemented to help direction therapy according to phenotypes, thereby improving outcomes [93]. Thus, patients with a musculoskeletal phenotype can be selected who most likely benefit from biofeedback interventions.

In patients with constipation, biofeedback therapy seems to benefit especially patients with dyssynergic defecation [21, 24, 28, 83, 86-88]. 
Another criterion of success might be a center's capacities to administer a certain amount of training sessions and the patient's willingness to complete the course of therapy as suggested by the therapist [19]. In patients with chronic constipation and dyssynergic defecation, consensus guidelines on biofeedback therapy [83] recommend 4-6 biofeedback sessions to manage dyssynergic defecation accordingly: 3 sessions [31] achieved a symptom improvement of only $45.3 \%$ compared to e.g. $80 \%$ achieved by 5 sessions in Chiarioni et al. [21], hence following existing consensus recommendations improves outcome. As biofeedback requires commitment on the patient's part to take responsibility for their own health, the patient's motivation and adequate encouragement to complete the course of therapy through the therapist are other important requirements for the therapeutic success [29, 36]. Cognitive impairment in the older population might lead to slower learning and the need for a higher number of treatment sessions [30]. Medical staff should be capable of demonstrating and explaining the method according to the patient's comprehension and education levels [36]. Similarly, counteracting problems of comprehension by using appro- priate explanations and psychological approaches are important in the work with children [54].

The effectiveness of pelvic floor biofeedback training also depends in part on the skills and experience of the biofeedback therapist and the particular techniques that are used to perform the training [28], which is why it is recommended to follow existing consensus guidelines [83].

As the access to biofeedback remains limited in many areas [31] and only a few centers offer biofeedback therapy, home-based self-training program is desirable and is a promising approach in anorectal and gynecologic (vulvar vestibulitis syndrome) disorders $[38,39,83]$, at least to continue training after initial training at a center.

With somatoform disorders and related syndromes, the etiology is still not fully understood but evidence supports an interaction of physiological, psychological and interpersonal factors [1]. Therefore, a multimodal treatment strategy can be promoted, using biofeedback, relaxation training and stress management to address physiological and emotional arousal as well as cognitive techniques, psychoeducation and attention training to alter cognitive-perceptual factors, a modification of illness behavior and graded
Fig. 1 Flow chart of the systematic literature search and the selection according to the Preferred Reporting Items for Systematic Reviews and Meta-Analyses (PRISMA) guidelines. UCPPS urological chronic pelvic pain syndrome

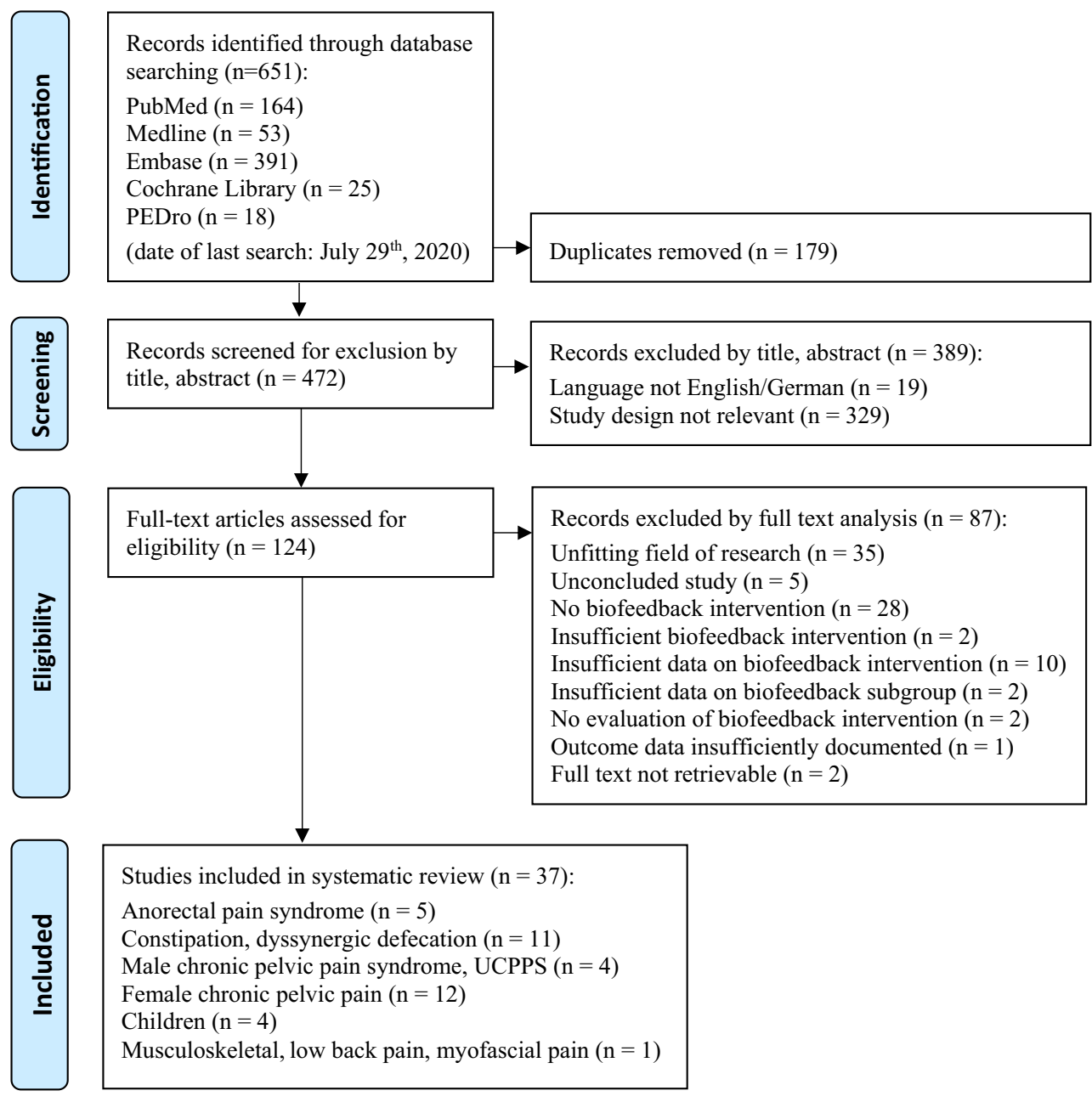




\section{review article}

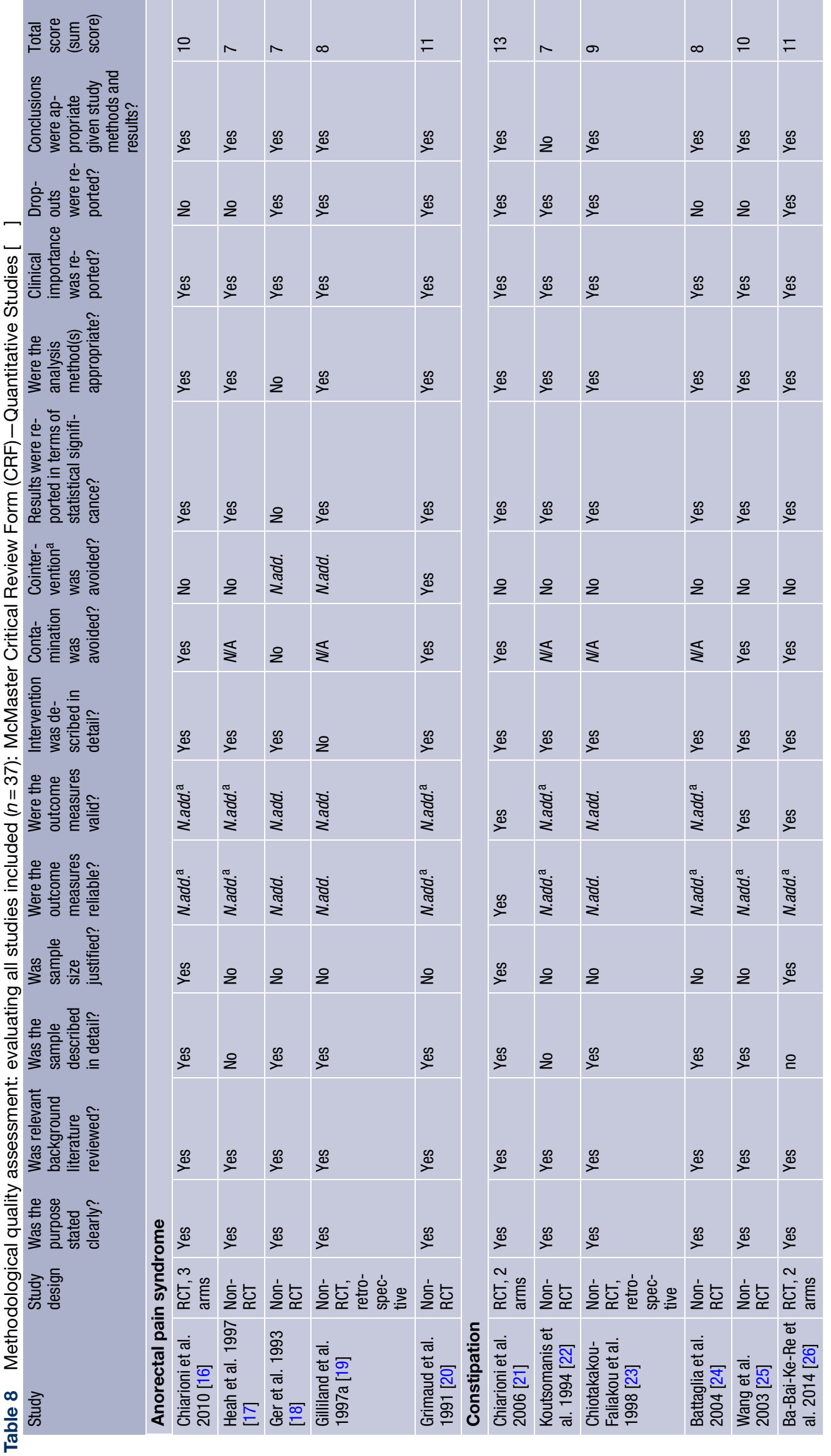




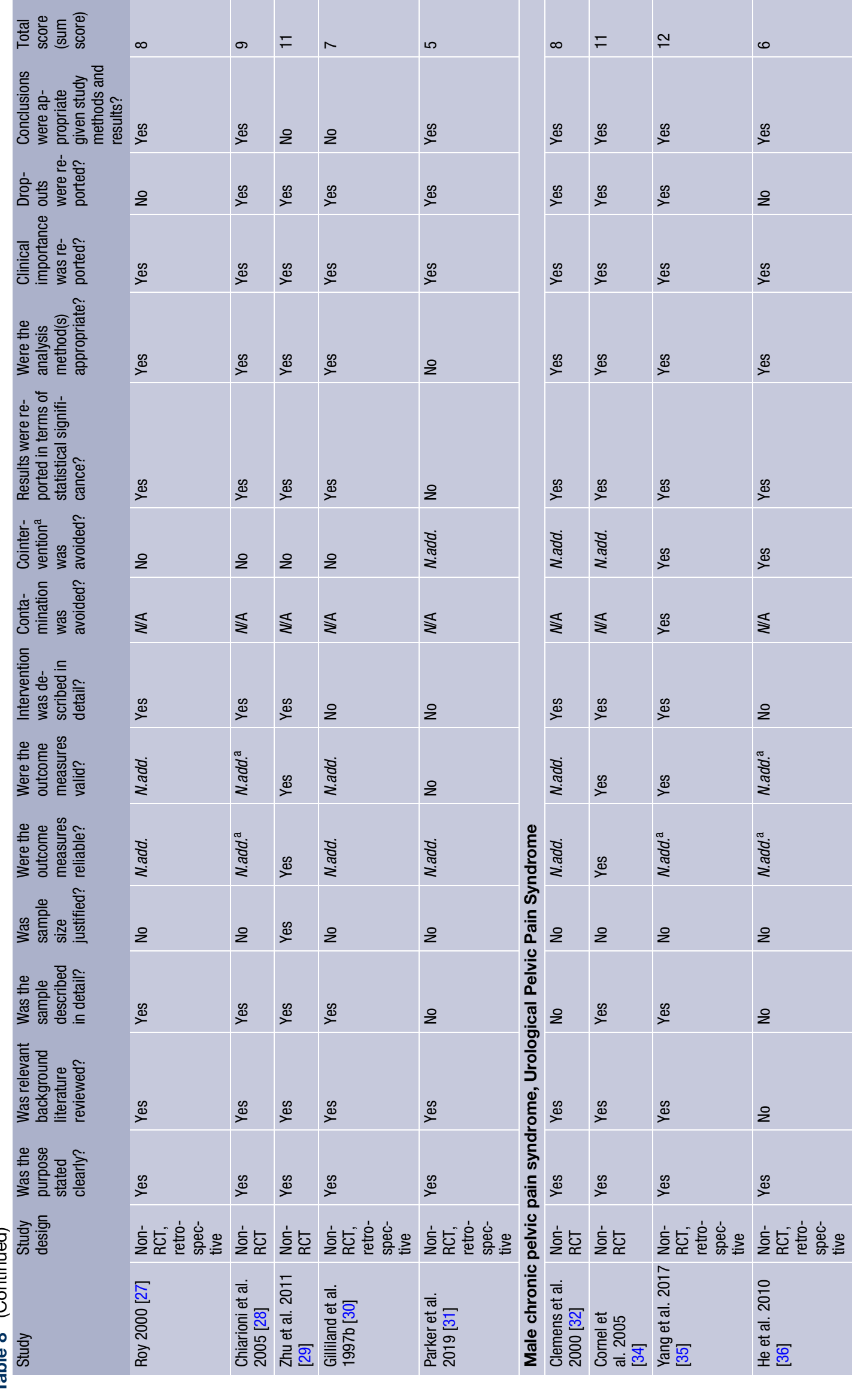




\begin{tabular}{|c|c|c|c|c|c|c|c|c|c|c|c|c|}
\hline 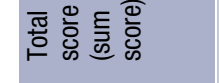 & $\sigma$ & n & 0 & $\simeq$ & 0 & $\infty$ & $\infty$ & $\sigma$ & $\simeq$ & $\circ$ & $=$ & $\simeq$ \\
\hline 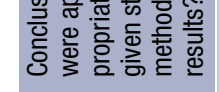 & $\stackrel{\infty}{\varnothing}$ & $\stackrel{\mathscr{L}}{\rightleftharpoons}$ & $\stackrel{\mathscr{B}}{\rightleftharpoons}$ & $\stackrel{\mathscr{J}}{\check{\nu}}$ & $\stackrel{\mathscr{D}}{\rightleftharpoons}$ & $\stackrel{\mathscr{B}}{\rightleftharpoons}$ & $\stackrel{2}{2}$ & Ф & $\stackrel{\mathscr{B}}{\rightleftharpoons}$ & $\stackrel{\mathscr{B}}{\not}$ & $\mathscr{\mathscr { D }}$ & $\stackrel{\infty}{\check{\nu}}$ \\
\hline & $\stackrel{\mathscr{\infty}}{\rightleftharpoons}$ & z & $\stackrel{\infty}{\infty}$ & 2 & $\stackrel{\mathscr{\Xi}}{=}$ & $\stackrel{2}{2}$ & $\stackrel{\mathscr{B}}{\rightleftharpoons}$ & $\stackrel{\mathscr{B}}{\rightleftharpoons}$ & $\stackrel{\mathscr{8}}{\rightleftharpoons}$ & $\stackrel{\mathscr{S}}{=}$ & $\stackrel{\mathscr{\infty}}{\rightleftharpoons}$ & $\stackrel{\mathscr{\infty}}{=}$ \\
\hline 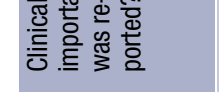 & $\stackrel{\mathscr{s}}{=}$ & $\stackrel{\mathscr{0}}{=}$ & $\stackrel{\mathscr{D}}{=}$ & $\stackrel{\mathscr{B}}{=}$ & $\stackrel{\infty}{=}$ & $\stackrel{\mathscr{B}}{\varnothing}$ & $\stackrel{\infty}{=}$ & $\stackrel{\mathscr{B}}{\rightleftharpoons}$ & $\stackrel{\mathscr{0}}{\infty}$ & $\stackrel{\mathscr{B}}{=}$ & $\stackrel{\infty}{\infty}$ & $\stackrel{\mathscr{8}}{=}$ \\
\hline 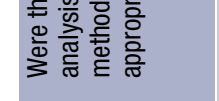 & $\stackrel{\mathscr{B}}{\rightleftharpoons}$ & $\stackrel{\mathscr{B}}{\longleftarrow}$ & $\stackrel{\mathscr{B}}{\rightleftharpoons}$ & $\stackrel{\mathscr{g}}{\rightleftharpoons}$ & $\stackrel{\mathscr{B}}{\varnothing}$ & $\stackrel{\mathscr{B}}{\rightleftharpoons}$ & 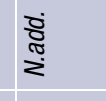 & 2 & $\stackrel{\mathscr{B}}{\rightleftharpoons}$ & $\stackrel{\mathscr{B}}{\rightleftharpoons}$ & $\stackrel{\mathscr{B}}{\rightleftharpoons}$ & $\stackrel{\mathscr{S}}{\rightleftharpoons}$ \\
\hline 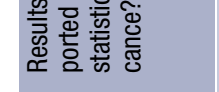 & $\stackrel{\mathscr{B}}{=}$ & $\stackrel{\mathscr{g}}{\rightleftharpoons}$ & $\stackrel{\mathscr{B}}{\infty}$ & $\stackrel{\mathscr{B}}{=}$ & $\stackrel{0}{\infty}$ & $\stackrel{\mathscr{B}}{\infty}$ & $\stackrel{\mathscr{B}}{=}$ & $\stackrel{\mathscr{B}}{>}$ & $\stackrel{\mathscr{B}}{=}$ & $\stackrel{\mathscr{S}}{=}$ & $\stackrel{\mathscr{B}}{\approx}$ & $\stackrel{\mathscr{s}}{=}$ \\
\hline & 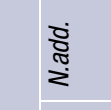 & $\stackrel{2}{2}$ & ঙ্ণ & $\stackrel{\mathscr{\infty}}{=}$ & ঙ্ণ & 密 & ঙ্ّ & $\stackrel{\infty}{\rightleftharpoons}$ & 2 & $\stackrel{2}{2}$ & 㱐 & 密 \\
\hline & $\underline{\Sigma}$ & 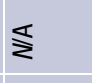 & $\stackrel{\Sigma}{z}$ & $\frac{\pi}{2}$ & s & $\stackrel{8}{\longleftarrow}$ & $\stackrel{8}{=}$ & $\dddot{\sharp}$ & $\stackrel{8}{\longleftarrow}$ & $\underline{z}$ & 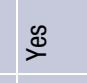 & 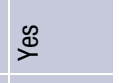 \\
\hline 5380 & $\stackrel{\mathscr{o}}{\rightleftharpoons}$ & $\stackrel{\mathscr{0}}{\rightleftharpoons}$ & $\stackrel{\mathscr{c}}{\check{x}}$ & $\stackrel{\mathscr{\infty}}{=}$ & $\stackrel{\infty}{=}$ & $\stackrel{8}{2}$ & $\stackrel{\mathscr{s}}{>}$ & $\stackrel{\mathscr{B}}{\not}$ & $\stackrel{\infty}{\rightleftharpoons}$ & $\stackrel{\mathscr{S}}{=}$ & $\stackrel{\mathscr{B}}{=}$ & $\stackrel{\infty}{\rightleftharpoons}$ \\
\hline 5 & $\stackrel{\mathscr{B}}{2}$ & 密 & ঙ্ّ & $\stackrel{\mathscr{B}}{\infty}$ & $\stackrel{2}{2}$ & ঙ্ّ & 密 & 密 & 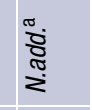 & $\stackrel{2}{2}$ & $\stackrel{\infty}{\infty}$ & 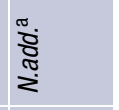 \\
\hline \े & 离 & 密 & ঙ্ّ & $\mathscr{\mathscr { B }}$ & 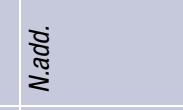 & 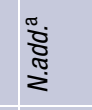 & 密 & 胥 & $\stackrel{\mathscr{B}}{\infty}$ & 密 & 密 & $\stackrel{\mathscr{S}}{x}$ \\
\hline & 2 & 2 & 2 & $\stackrel{\mathscr{g}}{\rightleftharpoons}$ & 2 & 2 & 2 & 2 & $\stackrel{8}{\longleftarrow}$ & $\stackrel{\infty}{\infty}$ & 2 & $\stackrel{\infty}{\infty}$ \\
\hline 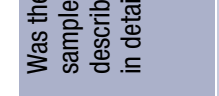 & $\stackrel{2}{2}$ & $\stackrel{\infty}{\sim}$ & $\stackrel{\infty}{=}$ & $\stackrel{\mathscr{B}}{=}$ & $\stackrel{\mathscr{0}}{=}$ & $\stackrel{\mathscr{\infty}}{\rightleftharpoons}$ & $\stackrel{\mathscr{\infty}}{=}$ & $\stackrel{2}{2}$ & $\stackrel{\mathscr{S}}{\infty}$ & $\stackrel{\mathscr{S}}{=}$ & $\stackrel{\infty}{\varnothing}$ & $\stackrel{\infty}{\nu}$ \\
\hline 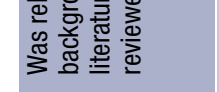 & $\stackrel{\mathscr{\infty}}{\rightleftharpoons}$ & $\stackrel{\infty}{=}$ & $\stackrel{\mathscr{s}}{\sim}$ & $\stackrel{\infty}{\rightleftharpoons}$ & $\stackrel{\mathscr{0}}{\varnothing}$ & $\stackrel{\mathscr{m}}{\rightleftharpoons}$ & $\stackrel{\mathscr{L}}{\rightleftharpoons}$ & $\stackrel{\mathscr{\infty}}{\rightleftharpoons}$ & $\stackrel{\mathscr{m}}{\rightleftharpoons}$ & $\stackrel{\mathscr{H}}{\rightleftharpoons}$ & $\stackrel{\mathscr{m}}{\rightleftharpoons}$ & $\stackrel{\mathscr{m}}{\rightleftharpoons}$ \\
\hline & 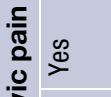 & $\stackrel{2}{2}$ & $\stackrel{\mathscr{c}}{>}$ & $\stackrel{\mathscr{s}}{\rightleftharpoons}$ & $\stackrel{\mathscr{B}}{>}$ & $\stackrel{\mathscr{m}}{\rightleftharpoons}$ & $\stackrel{\mathscr{M}}{\rightleftharpoons}$ & $\stackrel{\mathscr{B}}{\rightleftharpoons}$ & $\stackrel{\mathscr{B}}{\rightleftharpoons}$ & $\stackrel{\mathscr{m}}{\rightleftharpoons}$ & $\stackrel{\mathscr{\infty}}{\rightleftharpoons}$ & $\stackrel{\mathscr{\infty}}{\rightleftharpoons}$ \\
\hline & 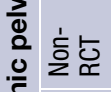 & 訔案 & iote & 㝘琼 & 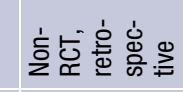 & t: & 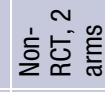 & 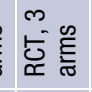 & 竞 & 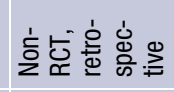 & 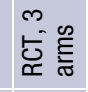 & 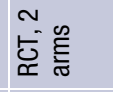 \\
\hline & 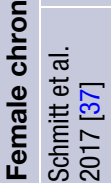 & 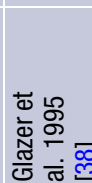 & 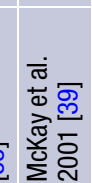 & 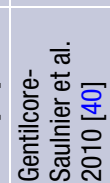 & 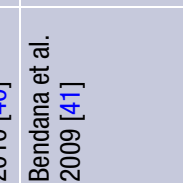 & 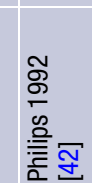 & 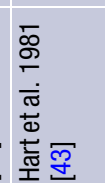 & 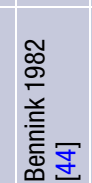 & 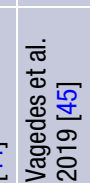 & 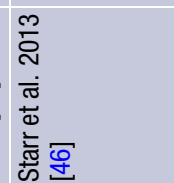 & 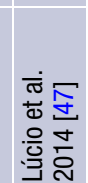 & 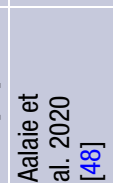 \\
\hline
\end{tabular}




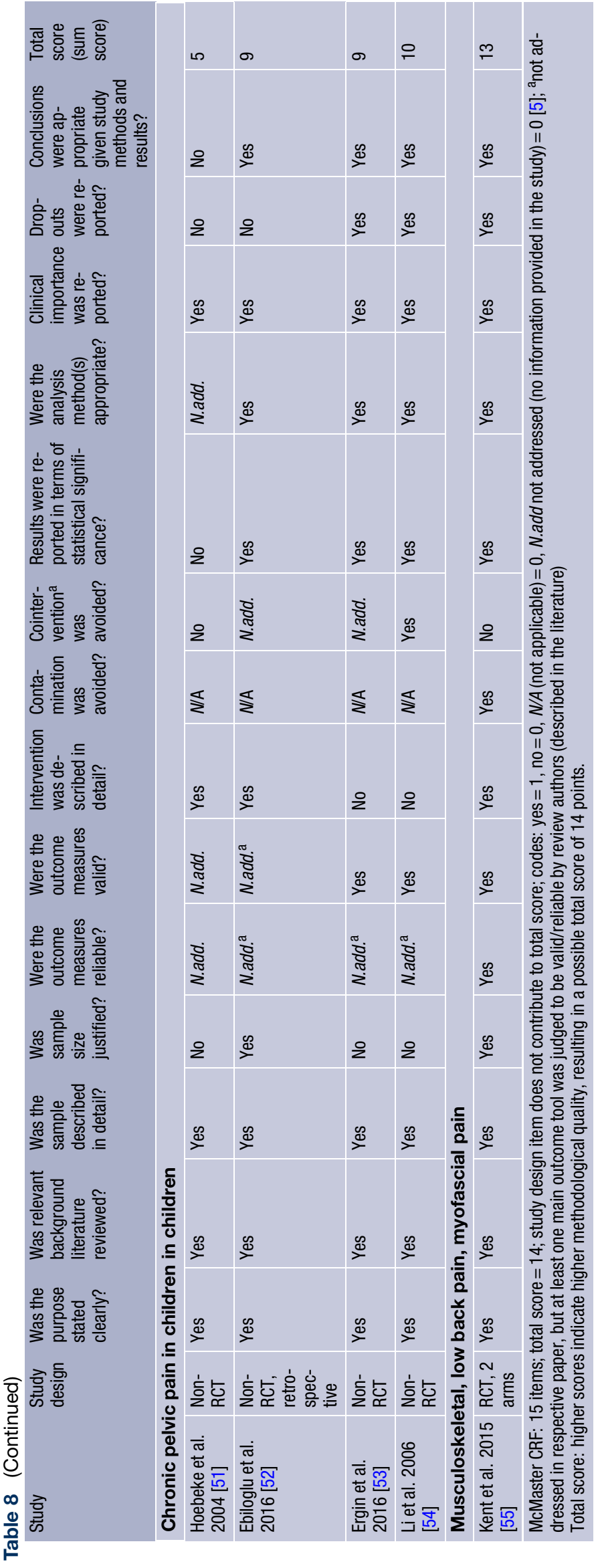


Table 9 Methodological quality assessment, evaluating the included randomized controlled trials $(n=9)$ : Physiotherapy Evidence Database (PEDro) scale [81]

\begin{tabular}{|c|c|c|c|c|c|c|c|c|c|c|c|c|c|}
\hline Criteria & $\begin{array}{l}\text { Eligibility } \\
\text { criteria and } \\
\text { source }\end{array}$ & $\begin{array}{l}\text { Random } \\
\text { allocation }\end{array}$ & $\begin{array}{l}\text { Concealed } \\
\text { allocation }\end{array}$ & $\begin{array}{l}\text { Baseline } \\
\text { compa- } \\
\text { rability }\end{array}$ & $\begin{array}{l}\text { Blinding } \\
\text { of sub- } \\
\text { jects }\end{array}$ & $\begin{array}{l}\text { Blinding } \\
\text { of ther- } \\
\text { apists }\end{array}$ & $\begin{array}{l}\text { Blinding } \\
\text { of as- } \\
\text { sessors }\end{array}$ & $\begin{array}{l}\text { Adequate } \\
\text { follow-up } \\
(>85 \%)\end{array}$ & $\begin{array}{l}\text { Intention- } \\
\text { to-treat } \\
\text { analysis }\end{array}$ & $\begin{array}{l}\text { Between- } \\
\text { group sta- } \\
\text { tistical } \\
\text { compar- } \\
\text { isons }\end{array}$ & $\begin{array}{l}\text { Reporting of } \\
\text { point mea- } \\
\text { sures and } \\
\text { measures of } \\
\text { variability }\end{array}$ & $\begin{array}{l}\text { Total } \\
\text { score }\end{array}$ & Quality \\
\hline $\begin{array}{l}\text { Chiarioni } \\
\text { et al. } 2010 \\
\text { [16] }\end{array}$ & Yes & Yes & Yes & Yes & No & No & Yes & Yes & Yes & Yes & Yes & 8 & Good \\
\hline $\begin{array}{l}\text { Chiarioni } \\
\text { et al. } 2006 \\
\text { [21] }\end{array}$ & Yes & Yes & Yes & Yes & No & No & Yes & No & No & Yes & Yes & 6 & Good \\
\hline $\begin{array}{l}\text { Ba-Bai-Ke- } \\
\text { Re et al. } \\
2014 \text { [26] }\end{array}$ & Yes & Yes & No & Yes & No & No & No & No & Yes & Yes & Yes & 5 & Fair \\
\hline $\begin{array}{l}\text { Philips et } \\
\text { al. } 1992 \\
\text { [42] }\end{array}$ & Yes & Yes & No & Yes & No & No & Yes & Yes & Yes & Yes & Yes & 7 & Good \\
\hline $\begin{array}{l}\text { Bennink et } \\
\text { al. } 1982 \\
{[44]}\end{array}$ & Yes & Yes & No & Yes & No & No & No & Yes & Yes & Yes & Yes & 6 & Good \\
\hline $\begin{array}{l}\text { Vagedes et } \\
\text { al. } 2019 \\
{[45]}\end{array}$ & Yes & Yes & Yes & Yes & No & No & No & No & Yes & Yes & Yes & 6 & Good \\
\hline $\begin{array}{l}\text { Lúcio et al. } \\
2014 \text { [47] }\end{array}$ & No & Yes & No & Yes & Yes & No & Yes & No & No & Yes & Yes & 6 & Good \\
\hline $\begin{array}{l}\text { Aalaie et al. } \\
2020 \text { [48] }\end{array}$ & Yes & Yes & Yes & Yes & No & No & No & Yes & Yes & Yes & Yes & 7 & Good \\
\hline $\begin{array}{l}\text { Kent et al. } \\
2015 \text { [55] }\end{array}$ & Yes & Yes & No & Yes & No & No & No & No & Yes & Yes & Yes & 5 & Fair \\
\hline
\end{tabular}

activity [1]. Multidisciplinary management, which is a common approach to many chronic conditions, is still not commonly available in gynecology because of cost factors and limited availability of interested specialists [7]. Yet multidisciplinary, multimodal and phenotype-oriented approaches have been increasingly proposed to deal with gynecologic phenotypes, such as provoked vestibulodynia and myofascial pain as well as with other chronic pelvic pain conditions such as bladder or prostate pain syndrome $[2,8,90$, 93].

Hence, biofeedback is not a complementary or alternative but an additive method for both diagnostic and therapeutic purposes. It should be used in addition to standard care, based on a state of the art concept, if the physician in charge gives the indication.

\section{Limitations of the present review}

The authors decided to include any quantitative study type of primary research to present a comprehensive overview of the current literature. This reduces the methodological quality of the trials and thereby the significance of the results.

The search term "pelvic pain" is wide-ranging, yet there are many terms used in literature to describe pain syndromes which are perceived in a certain organ [94] and specific pathologies that cause pelvic pain. Therefore, our pragmatic and generalized search strategy carries the risk of missing relevant articles. Studies evaluating biofeedback on constipated patients were included, yet constipation was not the primary focus of this paper as the pain component is not paramount; however, this phenotype has been researched in depth, and our search term did not reveal all relevant studies available in the literature. As with the phenotype of dyspareunia, the reader is referred to the respective relevant literature $[14,83$, 86, 90].

Due to language restrictions, studies that would have otherwise fulfilled the inclusion criteria could not be included.

\section{Conclusion}

Several landmark studies demonstrated the efficacy of biofeedback for anorectal disorders. For other phenotypes of chronic pelvic pain, there is tentative evidence that biofeedback-assisted training interventions can improve the outcomes on pain, overall symptoms, and quality of life. Clinical improvements came along with improvements in certain physiological parameters in several studies. Many trials were characterized by methodological limitations, such as a very small sample size, nonvalidated outcomes and 
a lack of control group. The preliminary positive findings should be investigated further in robust and well-designed randomized controlled trials. Certain factors have been identified that might be relevant for improving biofeedback treatment success.

\section{Implications for future research}

Future studies should aim to:

- conduct a systematic literature review using MeSH terms that more thoroughly evaluate the effect of biofeedback therapy in a certain phenotype (e.g. anorectal disorders, urological chronic pelvic pain syndrome, bladder pain syndrome, gynecologic pelvic pain conditions);

- list the term "pelvic pain" in the keywords of studies on certain pelvic pain subtypes so that these trials are detected by a literature search on the umbrella term (as chronic pelvic pain comprises many phenotypes);

- improve the quality of future studies, e.g. by choosing an RCT study design that is based on a sample size calculation, performing a post hoc analysis or a correction for multiple testing;

- report on the effect size and an estimate of their precision such as the confidence interval to describe the clinical relevance of results;

- conduct future trials with more homogeneous outcome assessment (to allow future meta-analysis). Ideally, validated questionnaires or pain scales should be used to measure outcome. For stating success rates, an international consensus on the graduation of these rating scales would be beneficial to standardize outcome and improve comparability between study results;

- continue to evaluate the optimum type and extent of biofeedback interventions for the certain phenotypes;

- evaluate changes in quality of life and psychological parameters, such as anxiety and depression (as psychological disorders are common comorbidities in patients with chronic pain conditions).

Funding Open access funding provided by the Medical University of Vienna.

Conflict of interest B. Wagner, M. Steiner, D.F.X. Huber and R. Crevenna declare that they have no competing interests.

Open Access This article is licensed under a Creative Commons Attribution 4.0 International License, which permits use, sharing, adaptation, distribution and reproduction in any medium or format, as long as you give appropriate credit to the original author(s) and the source, provide a link to the Creative Commons licence, and indicate if changes were made. The images or other third party material in this article are included in the article's Creative Commons licence, unless indicated otherwise in a credit line to the material. If material is not included in the article's Creative Commons licence and your intended use is not permitted by statutory regulation or exceeds the permitted use, you will need to obtain permis- sion directly from the copyright holder. To view a copy of this licence, visit http://creativecommons.org/licenses/by/4.0/.

\section{References}

1. Nanke A, RiefW. Biofeedback in somatoform disorders and related syndromes. Curr Opin Psychiatry. 2004;17(2):133-8.

2. EngelerD, Baranowski A, Berghmans B, Borovicka J, Cottrell A, Dinis-Oliveira $\mathrm{P}$, et al. EAU guidelines on chronic pelvic pain. 2019. https://uroweb.org/wp-content/uploads/ EAU-Guidelines-on-Chronic-Pelvic-Pain-2019.pdf. Accessed 28 Aug 2020, p. 1-86.

3. Latthe P, Latthe M, Say L, Gülmezoglu M, Khan KS. WHO systematic review of prevalence of chronic pelvic pain: a neglected reproductive health morbidity. BMC Public Health. 2006;6(1):177.

4. Ahangari A. Prevalence of chronic pelvic pain among women: an updated review. Pain Physician. 2014;17(2): E141-7.

5. Klotz SGR, Schön M, Ketels G, Löwe B, Brünahl CA. Physiotherapy management of patients with chronic pelvic pain (CPP): a systematic review. Physiother Theory Pract. 2019;35(6):516-32.

6. Krieger JN, Lee SWH, Jeon J, Cheah PY, Liong ML, Riley DE. Epidemiology of prostatitis. Int J Antimicrob Agents. 2008;31(Suppl 1):S85-S90.

7. Cheong YC, Smotra G, de Williams ACDC. Non-surgical interventions for the management of chronic pelvic pain. Cochrane Database Syst Rev. 2014; https://doi.org/10. 1002/14651858.CD008797.pub2.

8. Pastore EA, Katzman WB. Recognizing myofascial pelvic pain in the female patient with chronic pelvic pain. J Obstet Gynecol Neonatal Nurs. 2012;41(5):680-91.

9. Jarrell JF, Vilos GA, Allaire C, Burgess S, Fortin C, Gerwin R, et al. No. 164-consensus guidelines for the management of chronic pelvic pain. J Obstet Gynaecol Can. 2018;40(11):e747-87.

10. Franco JVAV, Turk T, Jung JH, Xiao Y-T, Iakhno S, Garrote $\mathrm{V}$, et al. Non-pharmacological interventions for treating chronic prostatitis/chronic pelvic pain syndrome. Cochrane Database Syst Rev. 2018; https://doi.org/10. 1002/14651858.CD012551.pub3.

11. Calhoun EA. The economic impact of chronic prostatitis. Arch Intern Med. 2004;164(11):1231.

12. Fuentes-Márquez P, Cabrera-Martos I, Valenza MC. Physiotherapy interventions for patients with chronic pelvic pain: a systematic review of the literature. Physiother Theory Pract. 2019;35(12):1131-8.

13. Loving S, Nordling J, Jaszczak P, Thomsen T. Does evidence support physiotherapy management of adult female chronic pelvic pain? A systematic review. Scand J Pain. 2012;3(2):70-81.

14. Koh CE, Young CJ, Young JM, Solomon MJ. Systematic review of randomized controlled trials of the effectiveness of biofeedback for pelvic floor dysfunction. Br J Surg. 2008;95(9):1079-87.

15. Liberati A, Altman DG, Tetzlaff J, Mulrow C, Gøtzsche PC, Ioannidis JPAA, et al. The PRISMA statement for reporting systematic reviews and meta-analyses of studies that evaluate health care interventions: explanation and elaboration. PLoS Med. 2009;6(7):e1000100.

16. Chiarioni G, Nardo A, Vantini I, Romito A, Whitehead WE. Biofeedback is superior to electrogalvanic stimulation and massage for treatment of levator ani syndrome. Gastroenterology. 2010;138(4):1321-9. 
17. Heah SM, Ho YH, Tan M, Leong AF. Biofeedback is effective treatment for levator ani syndrome. Dis Colon Rectum. 1997;40(2):187-9.

18. Ger GC, Wexner SD, Jorge JMN, Lee E, Amaranath LA, Heymen S, et al. Evaluation and treatment of chronic intractable rectal pain - a frustrating endeavor. Dis Colon Rectum. 1993;36(2):139-45.

19. Gilliland R, Heymen JS, Altomare DF, Vickers D, Wexner SD. Biofeedback for intractable rectal pain: outcome and predictors of success. Dis Colon Rectum. 1997a;40(2):190-6.

20. Grimaud JC, Bouvier M, Naudy B, Guien C, Salducci J. Manometric and radiologicinvestigations and biofeedback treatment of chronic idiopathic anal pain. Dis Colon Rectum. 1991;34(8):690-5.

21. Chiarioni G, Whitehead WE, Pezza V, Morelli A, Bassotti G. Biofeedback is superior to laxatives for normal transit constipation due to pelvic floor dyssynergia. Gastroenterology. 2006;130(3):657-64.

22. Koutsomanis D, Lennard-Jones JE, Kamm MA. Prospective study of biofeedback treatment for patients with slow and normal transit constipation. Eur J Gastroenterol Hepatol. 1994;6(2):131-7.

23. Chiotakakou-Faliakou E, Kamm MA, Roy AJ, Storrie JB, Turner IC. Biofeedback provides long-term benefit for patients with intractable, slow and normal transit constipation. Gut. 1998;42(4):517-21.

24. Battaglia E, Serra AM, Buonafede G, Dughera L, Chistolini F, Morelli A, et al. Long-term study on the effects of visual biofeedback and muscle training as a therapeutic modality in pelvic floor dyssynergia and slow-transit constipation. Dis Colon Rectum. 2004;47(1):90-5.

25. Wang J, Luo M-H, Qi Q-H, Dong Z-L. Prospective study of biofeedback retraining in patients with chronic idiopathic functional constipation. World J Gastroenterol. 2003;9(9):2109-13.

26. Ba-Bai-Ke-Re M-MT-JA, Wen N-R, Hu Y-L, Zhao L, Tuxun T, Husaiyin A, et al. Biofeedback-guided pelvic floor exercise therapy for obstructive defecation: an effective alternative. World JGastroenterol. 2014;20(27):9162-9.

27. Roy AJ, Emmanuel AV, Storrie JB, Bowers J, Kamm MA. Behavioural treatment (biofeedback) for constipation following hysterectomy. Br J Surg. 2000;87(1):100-5.

28. Chiarioni G, Salandini L, Whitehead WE. Biofeedback benefits only patients with outlet dysfunction, not patients with isolated slow transit constipation. Gastroenterology. 2005;129(1):86-97.

29. Zhu F-F, Lin Z, Lin L, Wang M-F. Changes in quality of life during biofeedback for people with puborectalis dyssynergia: generic and disease-specific measures. J Adv Nurs. 2011;67(6):1285-93.

30. Gilliland R, Heymen S, Altomare DF, Park UC, Vickers D, Wexner SD. Outcome and predictors of success of biofeedbackfor constipation. Br J Surg. 1997b;84(8):1123-6.

31. Parker CH, Henry S, Liu LWC. Efficacy of biofeedback therapy in clinical practice for the management of chronic constipation and fecal incontinence. JCan Assoc Gastroenterol. 2019;2(3):126-31.

32. Clemens JQ, Nadler RB, Schaeffer AJ, Belani J, Albaugh J, Bushman W. Biofeedback, pelvic floor re-education, and bladder training for male chronic pelvic pain syndrome. Urology. 2000;56(6):951-5.

33. Schaeffer AJ, Litwin MS, Krieger JN, Pontari M, Shoskes DA, Nadler RB, et al. Classification (traditional and national institutes of health) and demographics of prostatitis. Urology. 2002;60(6 SUPPL. A). https://doi.org/10.1016/s00904295(02)02292-6.
34. Cornel EB, Van Haarst EP, Browning-Groote Schaarsberg RWM, Geels J, Schaarsberg RWMB-G, Geels J. The effect of biofeedback physical therapy in men with chronic pelvic pain syndrome type III. Eur Urol. 2005;47(5):607-11.

35. Yang MH, Huang YH, Lai YF, Zeng SW, Chen SL. Comparing electromagnetic stimulation with electrostimulation plus biofeedback in treating male refractory chronic pelvic pain syndrome. Urol Sci. 2017;28(3):156-61.

36. He W, Chen M, Zu X, Li Y, Ning K, Qi L. Chronic prostatitis presenting with dysfunctional voiding and effects of pelvic floor biofeedback treatment. BJU Int. 2010;105(7):975-7.

37. Schmitt JJ, Singh R, Weaver AL, Mara KC, HarveySpringer RR, Fick FR, et al. Prospective outcomes of a pelvic floor rehabilitation program including vaginal electrogalvanic stimulation for urinary, defecatory, and pelvic pain symptoms. Female Pelvic Med Reconstr Surg. 2017;23(2):108-13.

38. Glazer HI, Rodke G, Swencionis C, Hertz R, Young AW. Treatment of vulvar vestibulitis syndromewith electromyographic biofeedback of pelvic floor musculature. J Reprod Med. 1995;40(4):283-90.

39. McKay E, Kaufman RH, Doctor U, Berkova Z, Glazer H, Redko V. Treating vulvar vestibulitis with electromyographic biofeedback of pelvic floor musculature. J Reprod Med. 2001;46(4):337-42.

40. Gentilcore-Saulnier E, McLean L, Goldfinger C, Pukall CF, ChamberlainS.Pelvicfloor muscleassessment outcomes in women with and without provoked vestibulodynia and the impact of a physical therapy program. J Sex Med. 2010;7(2 PART 2):1003-22.

41. Bendaña EE, Belarmino JM, Dinh JH, Cook CL, Murray BP, Feustel PJ, et al. Efficacy of transvaginal biofeedback and electrical stimulation in women with urinary urgency and frequency and associated pelvic floor muscle spasm. Urol Nurs. 2009;29(3):171-6.

42. Philips HC, Fenster HN, Samsom D. An effective treatment for functional urinary incoordination. J Behav Med. 1992;15(1):45-63.

43. Hart AD, Mathisen KS, Prater JS. A comparison of skin temperature and EMG training for primary dysmenorrhea. BiofeedbackSelf Regul. 1981;6(3):367-73.

44. Bennink CD, Hulst LL, Benthem JA. The effects of EMG biofeedback and relaxation training on primary dysmenorrhea. J Behav Med. 1982;5(3):329-41.

45. Vagedes J, FazeliA, BoeningA, HelmertE, BergerB, MartinD. Efficacy of rhythmical massage in comparison to heart rate variability biofeedback in patients with dysmenorrhea-A randomized, controlled trial. Complement Ther Med. 2019;42:438-44.

46. Starr JA, Drobnis EZ, Lenger S, Parrot J, Barrier B, Foster R. Outcomes of a comprehensive nonsurgical approach to pelvicfloor rehabilitation for urinarysymptoms, defecatory dysfunction, and pelvic pain. Female Pelvic Med Reconstr Surg. 2013;19(5):260-5.

47. Lúcio AC, D'Ancona CAL, Lopes MHBM, Perissinotto MC, Damasceno BP, Ancona CALD, et al. The effect of pelvic floor muscle training alone or in combination with electrostimulation in the treatment of sexual dysfunction in women with multiple sclerosis. Mult Scler J. 2014;20(13):1761-8.

48. Aalaie B, Tavana B, Rezasoltani Z, Aalaei S, Ghaderi J, Dadarkhah A. Biofeedback versus electrical stimulation for sexual dysfunction: a randomized clinical trial. Int Urogynecol J. 2020; https://doi.org/10.1007/s00192-02004373-7.

49. Maseroli E, Scavello I, Rastrelli G, Limoncin E, Cipriani S, Corona G, et al. Outcome of medical and psychosexual interventions for vaginismus: a systematic review and 
meta-analysis. J Sex Med. 2018;15. https://doi.org/10. 1016/j.jsxm.2018.10.003.

50. Pacik PT, Geletta S. Vaginismus treatment: clinical trials followup 241 patients. SexMed. 2017;5(2):e114-e23.

51. Hoebeke P, Van Laecke E, Renson C, Raes A, Dehoorne J, Vermeiren $\mathrm{P}$, et al. Pelvic floor spasms in children: an unknown condition responding well to pelvic floor therapy. Eur Urol. 2004;46(5):651-4.

52. Ebiloglu T, Kaya E, Köprü B, Topuz B, Irkilata HC, Kibar Y. Biofeedback as a first-line treatment for overactive bladder syndrome refractory to standard urotherapy in children. JPediatr Urol. 2016;12(5):290.e1-290.e7.

53. Ergin G, Kibar Y, Ebiloğlu T, Irkilata HC, Kopru B, Kaya E, et al. The role of urinary nerve growth factor for the diagnosis and assessment of the biofeedback success in children with dysfunctional voiding. J Pediatr Urol. 2016;12(2):118.e1-118.e6.

54. LiY, Qi L, Wen JG, ZuXB, Chen ZY. Chronic prostatitis during puberty. BJUInt. 2006;98(4):818-21.

55. Kent P, Laird R, Haines T. The effect of changing movementand posture using motion-sensor biofeedback, versus guidelines-based care, on the clinical outcomes of people with sub-acute or chronic low back pain-a multicentre, cluster-randomised, placebo-controlled, pilot trial. BMC Musculoskelet Disord. 2015. https://doi.org/10.1186/ s12891-015-0591-5.

56. Neijenhuijs KI, Hooghiemstra N, HoltmaatK, Aaronson NK, Groenvold M, Holzner B, et al. The female sexual function index (FSFI) - A systematic review of measurement properties. J Sex Med. 2019;16. https://doi.org/10.1016/j.jsxm. 2019.03.001.

57. Akbal C, Şahan A, Şener TE, Şahin B, Tinay I, Tarcan T, et al. Diagnostic value of the pediatric lower urinary tract symptom score in children with overactive bladder. World J Urol. 2014;32(1):201-8.

58. Chesney MA, Tasto DL. The development of the menstrual symptom questionnaire. Behav Res Ther. 1975;13(4). https://doi.org/10.1016/0005-7967(75)90028-5.

59. American Urological Association (AUA). Symptom score questionnaire. 2016 Boston Scientific Corporation or its affiliates. September 2012. https://www.bostonscientific. com/content/dam/bostonscientific-anz/patients/down loads/Enlarged_Prostate_Symptom_Score_Questionnaire. pdf. Accessed 23 Feb 2021.

60. Karcioglu O, Topacoglu H, Dikme O, Dikme O. A systematic review of the pain scales in adults: which to use? Am JEmerg Med. 2018;36(4):707-14.

61. Cohen J.Statistical power analsis of the behavioral sciences. NewJersey: LawrenceEarlbaum; 1988

62. Agachan F, Chen T, Pfeifer J, Reissman P, Wexner SD. A constipation scoring system to simplify evaluation and management of constipated patients. Dis Colon Rectum. 1996;39(6):681-5.

63. Sawilowsky SS. New effect size rules of thumb. J Mod Appl Stat Methods. 2009. https://doi.org/10.22237/JMASM/ 1257035100

64. Akbal C, Genc Y, Burgu B, Ozden E, Tekgul S. Dysfunctional voiding and incontinence scoring system: Quantitative evaluation of incontinence symptoms in pediatric population. JUrol. 2005;173(3):969-73.

65. Selekman RE, Harris CR, Filippou P, Chi T, Alwaal A, Blaschko SD, et al. Validation of a visual prostate symptom score in men with lower urinary tract symptoms in a health safety net hospital. Urology. 2015;86(2):354-8.

66. Clemens JQ, Calhoun EA, Litwin MS, McNaughtonCollins M, Dunn RL, Crowley EM, et al. Rescoring the NIH chronic prostatitis symptom index: nothing new. Prostate Cancer Prostatic Dis. 2009;12(3):285-7.

67. Ware JE, Sherbourne CD. The MOS 36-item short-form health survey (Sf-36): I. conceptual framework and item selection. Med Care. 1992;30(6):473-83.

68. Resnick B, Parker R. Simplified scoring and psychometrics of the revised 12-item short-form health survey. Outcomes Manag Nurs Pract. 2001;5(4):161-6.

69. Laycock J, Jerwood D. Pelvic floor muscle assessment: the PERFECT scheme. Physiotherapy. 2001;87(12):631-42.

70. Gonnermann A, Framke T, Großhennig A, Koch A. No solution yet for combining two independent studies in the presence of heterogeneity. Stat Med. 2015;34(16):2476-80.

71. Popay J, Roberts H, Sowden A, Petticrew M, Arai L, Rodgers $\mathrm{M}$, et al. Guidance on the conduct of narrative synthesis in systematic reviews. 2006. http://citeseerx.ist.psu. edu/viewdoc/download?doi=10.1.1.178.3100\&rep=rep1\& type $=$ pdf. Accessed 7 June 2020 .

72. Law M, Stewart D, Pollock N, Letts L, Bosch J, Westmorland M. Critical review form-quantitative studies. 1998. https://www.unisa.edu.au/siteassets/episerver6-files/global/health/sansom/documents/icahe/cats/ mcmasters_quantitative-review.pdf. Accessed 23 February 2021.

73. Law M, Stewart D, Pollock N, Letts L, Bosch J, Westmorland M. Guidelines for critical review form-quantitative studies. 1998. https://canchild.ca/system/tenon/ assets/attachments/000/000/366/original/quantguide. pdf?license=yes. Accessed 23 February 2021

74. Briggs AM, Smith AJ, Straker LM, Bragge P. Thoracic spine pain in the general population: prevalence, incidence and associated factors in children, adolescents and adults. Asystematic review. BMCMusculoskeletDisord. 2009;10(1):77.

75. Daly AE, Bialocerkowski AE. Does evidence support physiotherapy management of adult Complex Regional Pain Syndrome Type One? A systematic review. Eur J Pain. 2009;13(4):339-53.

76. Maher CG, Sherrington C, Herbert RD, Moseley AM, Elkins M. Reliability of the PEDro scale for rating quality of randomized controlled trials. Phys Ther. 2003;83(8):713-21.

77. De Morton NA. The PEDro scale is a valid measure of the methodological quality of clinical trials: a demographic study. Aust J Physiother. 2009;55(2):129-33.

78. Bhogal SK, Teasell RW, Foley NC, Speechley MR. The PEDro scale provides a more comprehensive measure of methodological quality than the Jadad scale in stroke rehabilitation literature. JClin Epidemiol. 2005;58(7):668-73.

79. Yamato TP, Maher C, Koes B, MoseleyA. The PEDro scalehad acceptably high convergent validity, construct validity, and interrater reliability in evaluating methodological quality of pharmaceutical trials. JClin Epidemiol. 2017;86:176-181

80. Cashin AG, McAuley JH. Clinimetrics: physiotherapy evidence database (PEDro) scale. J Physiother. 2020;66:59.

81. Physiotherapy Evidence Database. PEDro Scale. 1999. https://pedro.org.au/wp-content/uploads/PEDro_scale. pdf. Accessed 23 Feb 2021.

82. Physiotherapy Evidence Database. PEDro. 2020. https:// www.pedro.org.au/. Accessed 14 July 2020.

83. Rao SSC, Benninga MA, Bharucha AE, Chiarioni G, Di Lorenzo C, Whitehead WE. ANMS-ESNM position paper and consensus guidelines on biofeedback therapy for anorectal disorders. Neurogastroenterol Motil. 2015;27(5):594-609.

84. TheAssociation for Applied PsychophysiologyandBiofeedback. Standards for performing biofeedback. 2020. https:// 
www.aapb.org/i4a/pages/index.cfm?pageid=3678. Accessed 7 Sept 2020.

85. Griffiths P, Needleman J. Statistical significance testing and p-values: defending the indefensible? A discussion paper and position statement. Int J Nurs Stud. 2019. https:// doi. org/10.1016/j.ijnurstu.2019.07.001.

86. Narayanan SP, Bharucha AE. A practical guide to biofeedback therapy for pelvic floor disorders. Curr Gastroenterol Rep. 2019;21(5):21.

87. Rao SSC, Seaton K, Miller M, Brown K, Nygaard I, Stumbo P, et al. Randomized controlled trial of biofeedback, sham feedback, and standard therapy for dyssynergic defecation. Clin Gastroenterol Hepatol. 2007. https:// doi.org/10.1016/ j.cgh.2006.12.023.

88. Heymen S, ScarlettY, Jones K, Ringel Y, Drossman D, Whitehead WE. Randomized, controlled trial shows biofeedback to be superior to alternative treatments for patients with pelvic floor dyssynergia-type constipation. Dis Colon Rectum. 2007;50(4):428-41.

89. Mariani L. Vulvar vestibulitis syndrome: an overview of non-surgical treatment. Eur J Obstet Gynecol Reprod Biol. 2002;101(2):109-12.

90. Morin M, Carroll M-SS, Bergeron S. Systematic review of the effectiveness of physical therapy modalities in women with provoked vestibulodynia. Sex Med Rev. 2017;5(3):295-322.
91. Bergeron S, Binik YM, Khalifé S, Pagidas K, Glazer HI, Meana $\mathrm{M}$, et al. A randomized comparison of group cognitive-behavioral therapy, surface electromyographic biofeedback, and vestibulectomy in the treatment of dyspareunia resulting from vulvar vestibulitis. Pain. 2001;91(3):297-306.

92. Shoskes DA, Nickel JC, Rackley RR, Pontari MA. Clinical phenotyping in chronic prostatitis/chronic pelvic pain syndrome and interstitial cystitis: a management strategy forurologic chronic pelvicpain syndromes. ProstateCancer Prostatic Dis. 2009;12(2):177-83.

93. Shoskes DA, Nickel JC, Kattan MW. Phenotypically directed multimodal therapy for chronic prostatitis/chronic pelvic pain syndrome: a prospective study using UPOINT. Urology. 2010. https://doi.org/10.1016/j.urology.2010.01.021.

94. Fall M, Baranowski AP, Elneil S, Engeler D, Hughes J, Messelink EJ, et al. EAU guidelines on chronic pelvic pain. Eur Urol. 2010;57(1):35-48.

Publisher's Note Springer Nature remains neutral with regard to jurisdictional claims in published maps and institutional affiliations. 